\title{
Origin and evolutionary plasticity of the gastric caecum in sea urchins (Echinodermata: Echinoidea)
}

\author{
Alexander Ziegler ${ }^{1 *}$, Rich Mooi $^{2}$, Gauthier Rolet ${ }^{3}$, Chantal De Ridder ${ }^{3}$
}

\begin{abstract}
Background: The digestive tract of many metazoan invertebrates is characterized by the presence of caeca or diverticula that serve secretory and/or absorptive functions. With the development of various feeding habits, distinctive digestive organs may be present in certain taxa. This also holds true for sea urchins (Echinodermata: Echinoidea), in which a highly specialized gastric caecum can be found in members of a derived subgroup, the Irregularia (cake urchins, sea biscuits, sand dollars, heart urchins, and related forms). As such a specialized caecum has not been reported from "regular" sea urchin taxa, the aim of this study was to elucidate its evolutionary origin.

Results: Using morphological data derived from dissection, magnetic resonance imaging, and extensive literature studies, we compare the digestive tract of 168 echinoid species belonging to 51 extant families. Based on a number of characters such as topography, general morphology, mesenterial suspension, and integration into the haemal system, we homologize the gastric caecum with the more or less pronounced dilation of the anterior stomach that is observed in most "regular" sea urchin taxa. In the Irregularia, a gastric caecum can be found in all taxa except in the Laganina and Scutellina. It is also undeveloped in certain spatangoid species.

Conclusions: According to our findings, the sea urchin gastric caecum most likely constitutes a synapomorphy of the Euechinoidea. Its occurrence in "regular" euechinoids is linked to the presence of an additional festoon of the anterior stomach in ambulacrum III. Both structures, the additional festoon and the gastric caecum, are absent in the sister taxon to the Euechinoidea, the Cidaroida. Since the degree of specialization of the gastric caecum is most pronounced in the predominantly sediment-burrowing irregular taxa, we hypothesize that its evolution is closely linked to the development of more elaborate infaunal lifestyles. We provide a comprehensive study of the origin and evolutionary plasticity of a conspicuous digestive tract structure, the gastric caecum, in a major taxon of the extant invertebrate macrozoobenthos.
\end{abstract}

\section{Background}

With few exceptions, metazoans possess an alimentary canal comprising a sac- or tube-like invagination of the body wall. The evolution of an internalized intestinal tract offered the possibility of digesting larger food particles [1]. The digestive system may form a simple or ramified cavity with a single aperture (as in the Cnidaria and the Platyhelminthes) or a tube with openings at its two ends that constitute a distinct mouth and anus, allowing the food to pass in one direction through a

\footnotetext{
* Correspondence: alexander.ziegler@charite.de

${ }^{1}$ Institut für Immungenetik, Charité-Universitätsmedizin Berlin, Thielallee 73, 14195 Berlin, Germany

Full list of author information is available at the end of the article
}

tubular system [2]. Subsequently, this has led to the specialization of entire digestive tract regions. The invertebrate gut can be subdivided into three major parts: the foregut (usually comprising mouth, pharynx, and esophagus), the midgut (crop, gizzard, and stomach), and the hindgut (intestine, rectum, and anus) [3]. In most taxa, the midgut serves as the primary site of digestion as well as nutrient absorption and is therefore often characterized by the presence of glands and caeca that serve secretory or absorptive functions.

In the context of the general pattern described above for typical bilaterian animals, it is important to note that even secondarily radial forms such as echinoderms tend to follow the same overall model of gut organization.

\section{Biomed Central}


Among the Echinodermata - a taxon of marine invertebrate deuterostomes - sea urchins (Echinoidea) are considered one of the best studied groups and serve as model organisms for a wide range of biological disciplines. The digestive tract of echinoids is usually subdivided into mouth, buccal cavity, pharynx, esophagus, stomach, intestine, rectum, and anus [4-6], with the mouth forming the proximal and the anus the distal segments. However, not all sea urchin taxa possess all of these gut sections and some are characterized by the presence of additional digestive tract structures such as festoons, siphons, Gregory's diverticulum, an intestinal caecum, or a gastric caecum [7]. The gastric caecum is a conspicuous organ that was first described by C.K. Hoffmann [8] in Spatangus purpureus, a species within the derived Spatangoida (Figure 1A), a monophyletic taxon

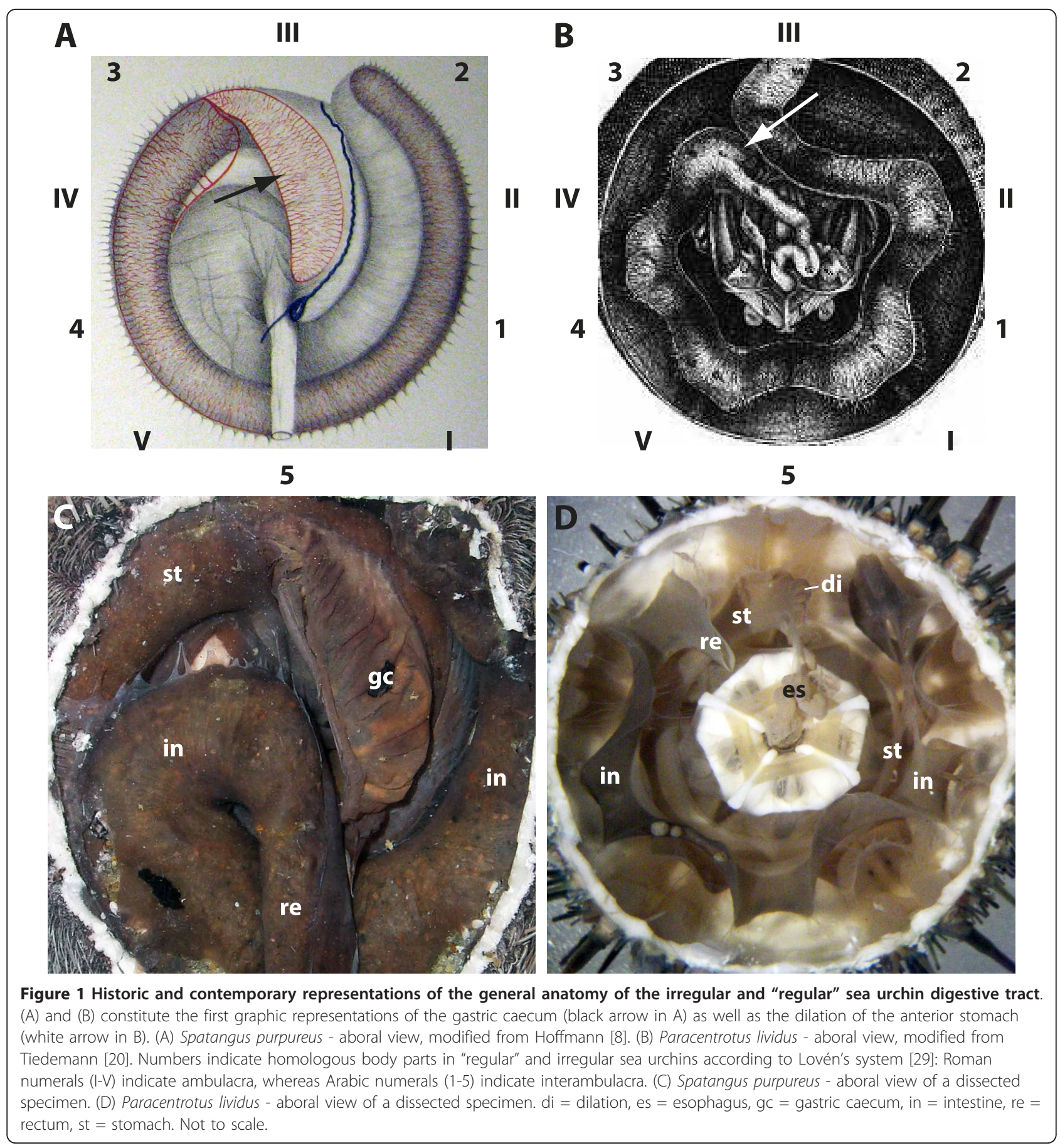


of irregular sea urchins characterized by an infaunal lifestyle. Several spatangoid taxa have been shown to possess this large, non-contractile pouch that is connected to the anterior stomach through a slit-like opening (Figure $1 \mathrm{C}$ ). This pouch is also well-connected to the haemal system through numerous haemal ducts within its connective tissue layer [9-12]. A number of hypotheses regarding the function of this structure in spatangoids have been presented. Some authors believed it to be a glandular organ whose secretions leak into the stomach [13], an absorptive structure [12,14], a site of microbial fermentation $[15,16]$, or simply an organ that acts generally in digestion $[17,18]$.

In contrast, the digestive tract in "regular" sea urchins is not characterized by the presence of such a highly specialized structure (the "regular" echinoids do not form a monophyletic group, hence the quotes; in contrast, the Irregularia is a recognized monophyletic taxon [19]). However, several authors [5,9,14,20-24] reported a more or less developed dilation at the proximal part of the anterior stomach in certain "regular" sea urchin taxa (Figure 1B, D). According to most authors, this dilation in "regular" sea urchins did not display any functional specialization and was therefore seen merely as a lateral outcrop of the stomach $[5,9,22,23]$. R. Koehler - who had systematically studied sea urchin internal anatomy - was presumably the first and so far the only author to briefly mention the potential homology of the dilation observed in "regular" sea urchins with the highly specialized gastric caecum found in the infaunal spatangoids and other irregular taxa in which the caecum had been described [9]. However, the precise evolutionary relationship between these structures has not yet been systematically elucidated, largely because a comprehensive analysis encompassing all major sea urchin taxa was not possible due to the lack of data. In addition, the multitude of terms assigned by several authors to the observed dilation of the sea urchin anterior stomach as well as the gastric caecum in the Irregularia has greatly complicated matters by obfuscating direct comparisons among observed occurrences (Table 1).

In order to provide an example for the evolutionary plasticity of invertebrate digestive tract structures, we here describe the diversity observed in the morphology of the sea urchin anterior stomach by investigating taxa representing a wide diversity of forms within the Echinoidea. The aim of our study was (i) to catalogue the diversity of the anterior stomach morphology observed among sea urchins, (ii) to suggest a number of homology criteria that apply to the observed structures in all sea urchin taxa included in our analysis, (iii) to elucidate the evolutionary origin of the highly specialized gastric caecum found in the derived Spatangoida, and (iv) to evaluate implications for sea urchin phylogeny. Using magnetic resonance imaging (MRI) and three-dimensional (3D) reconstruction in combination with dissection and an extensive literature survey, we were able to incorporate 168 sea urchin species belonging to 51 extant families into our analysis (Figure 2). This comprehensive survey will serve as a basis for future studies involving the ecology, histology, ultrastructure, and function of digestive tract structures in a major taxon of the invertebrate macrozoobenthos.

\section{Results}

The following descriptions give an overview of the anterior stomach found in 51 echinoid families (Figure 2). We focus here on the general location of this part of the digestive tract within members of each family as well as the presence or absence of sub-structures. Intraand inter-specific variability exists for certain internal

Table 1 Trilingual list of terms assigned to the pouch encountered in irregular as well as to the dilation of the anterior stomach observed in "regular" sea urchin species by various authors

\begin{tabular}{|c|c|c|c|}
\hline English & & French & German \\
\hline actinal intestinal appendage & diverticulum of the stomach & appendice & Blinddarm \\
\hline anterior caecum & festoon & appendice cecal & Blindsack \\
\hline blind diverticulum & first caecum & caecum & Blindsackbildung \\
\hline blind gut & gastric caecum & caecum gastrique & Caecum \\
\hline blindsac & intestinal appendage & caecum stomacal & Coecum \\
\hline blind sac & pouch & coecum stomacal & Erstes Divertikel \\
\hline caecum & $\mathrm{sac}$ & cul-de-sac & Divertikel \\
\hline coecum & sac-like dilatation & cul-de-sac antérieur de l'intestin & Erweiterung \\
\hline digestive caecum & sac-like swelling & diverticule en cul-de-sac & \\
\hline dilatation & stomach caecum & diverticulum & \\
\hline dilation & swelling & diverticulum intestinal & \\
\hline diverticulum & & glande intestinale & \\
\hline
\end{tabular}

Listed in alphabetical order. 


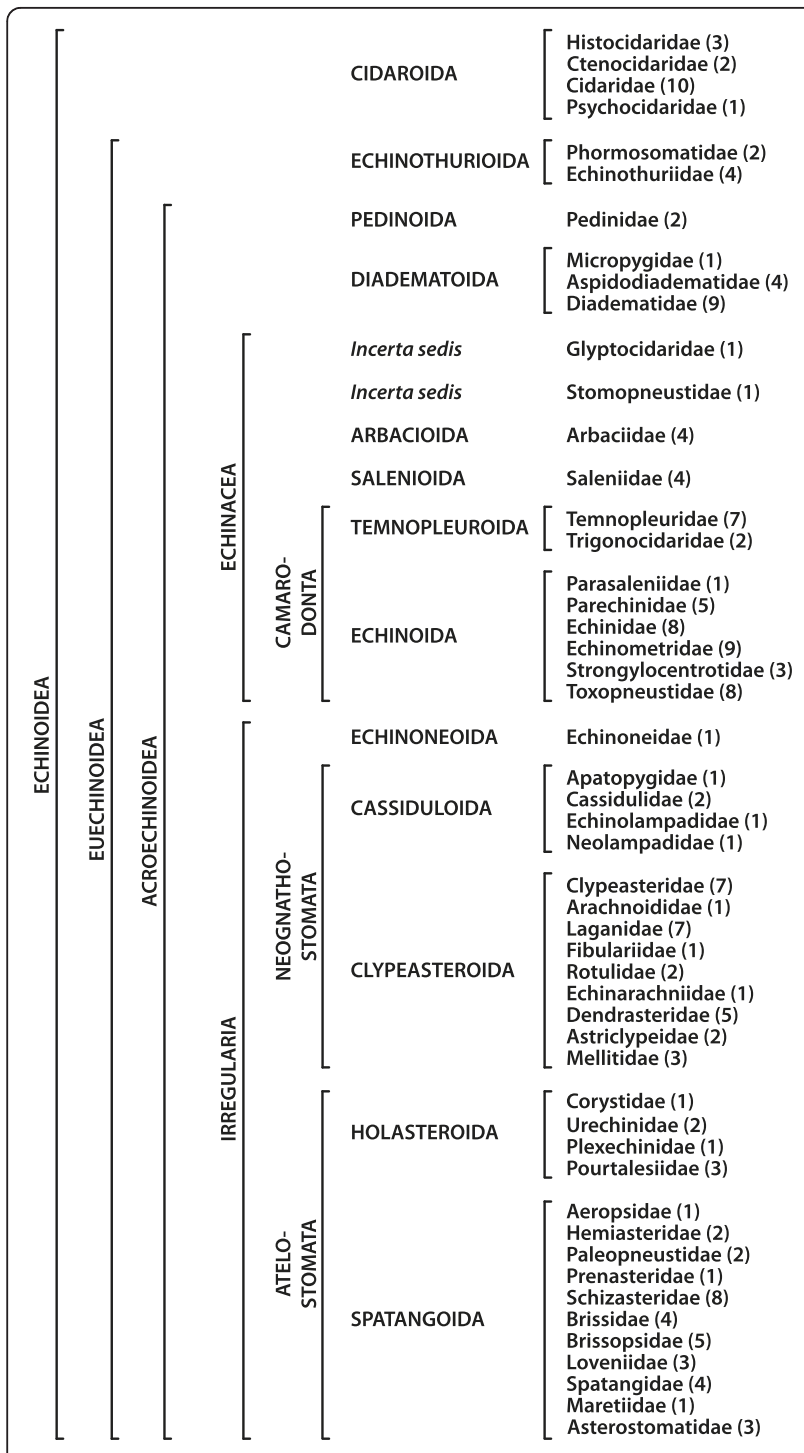

Figure 2 List of higher sea urchin taxa analyzed in this study. Note that the monophyly of several of these taxa is still under debate. The numbers in brackets designate the number of species analyzed in each family in the course of this study. This diagram is based upon results obtained by numerous authors [74-84].

structures in sea urchins [25]. Therefore, we only mention the relevant deviations from our general findings at the family level. We regard the anterior stomach as beginning immediately distal to the junction of esophagus and stomach in the vicinity of the branching-off point of the primary siphon (some irregular taxa possess a secondary siphon [5]). A certain degree of histological specialization is known to exist in the anterior part of the stomach in "regular" taxa $[24,26,27]$. The primary siphon, although a derivative of the entire stomach and therefore also present in the anterior stomach, is not considered here, primarily because histological techniques not used in this study have been shown to be essential in determining presence or absence of the primary siphon [28].

The topographic reference system for our descriptions is based upon Lovén's system [29] as depicted in Figure $1 \mathrm{~A}, \mathrm{~B}$ [ambulacra I-V (Amb I-V) and interambulacra 15 (IAmb 1-5)]. Furthermore, Figures 3, 4, 5, 6, 7, 8 denote whether the specimen is viewed aborally $(A B)$, laterally $(L A)$, or orally $(O R)$. Figure 9 provides an overview of the general sea urchin digestive tract morphology - the models presented in this figure are entirely based on 3D MRI datasets [25,30]. Finally, Figure 10 provides three interactive $3 \mathrm{D}$ models of the digestive tracts of selected taxa. In all figures within the present article, except for a number of lateral views, Amb III is always facing upwards. The images taken from the literature have in some cases been modified slightly through removal of labels used by the original author(s). All images were chosen based on the quality and plausibility in the manner in which digestive tract structures in particular had been depicted.

Specimens were aligned according to Lovén's system by first locating the axial complex within the specimen. The axial complex is a structure formed by various primary and secondary body cavities which is located in IAmb 2 underneath the madreporic plate - see [31] for a survey of this structure within the Echinoidea. Tables 2 and 3 provide information on all species analyzed within this study, in particular on the method forming the basis of the description (i.e. dissection, MRI, or literature references [32-70]).

\section{"Regularia"}

The digestive tract of "regular" sea urchin species consists of two loops that lie more or less on top of each other and usually bear so-called festoons, i.e. vertical inflections of the gut (Figure 9A-K).

\section{Histocidaridae}

The anterior stomach of Histocidaris elegans, Histocidaris variabilis (Figure 3A), and Poriocidaris purpurata is located in Amb III. The slightly curving esophagus is initially directed towards Amb III. The anterior stomach spans Amb III horizontally and is composed of a single festoon. A small dilation extends adapically immediately distal to the junction of esophagus and stomach.

\section{Ctenocidaridae}

The morphology of the anterior stomach in Ctenocidaris nutrix and Notocidaris gaussensis closely resembles that found in the Histocidaridae. The anterior stomach spans Amb III horizontally close to its connection with the esophagus, and is composed of a single festoon. A small dilation extends adapically immediately distal to the junction of esophagus and stomach. 


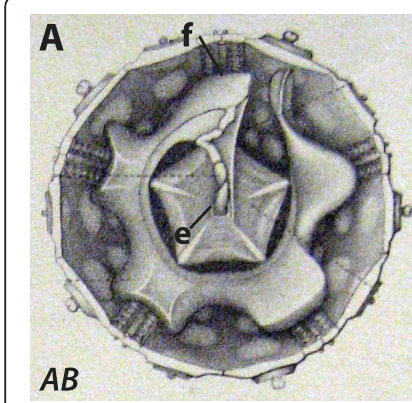

Histocidaris variabilis (from [32])

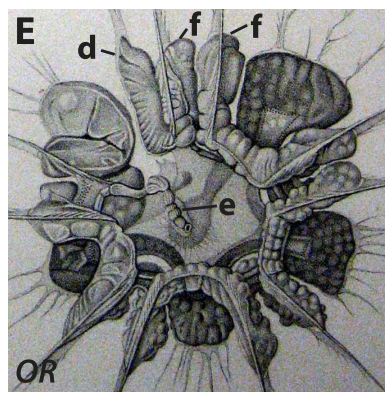

Sperosoma obscurum (from [35])

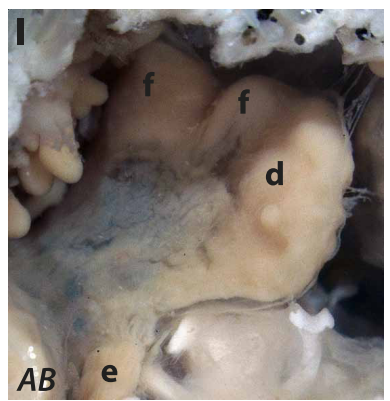

Caenopedina mirabilis (USNM 31178)

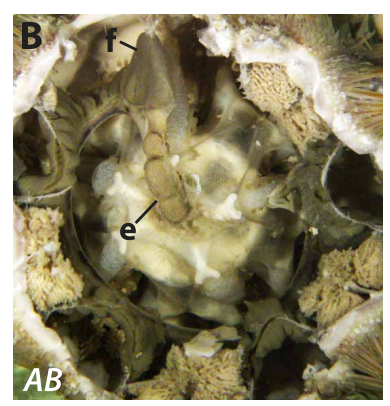

Cidaris cidaris (this study)

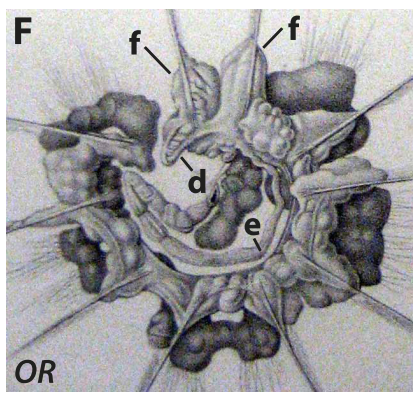

Tromikosoma hispidum (from [35])

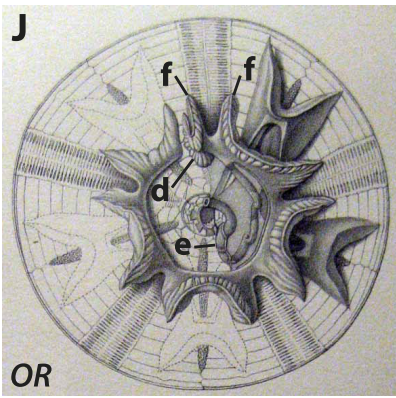

Micropyga tuberculata (from [35])

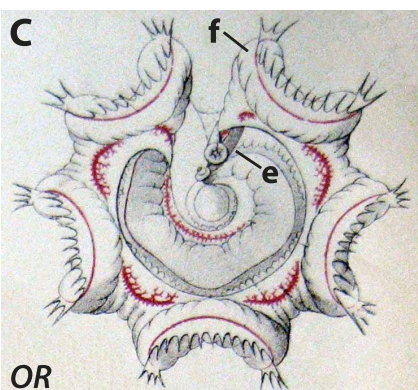

Cidaris cidaris (from [33])

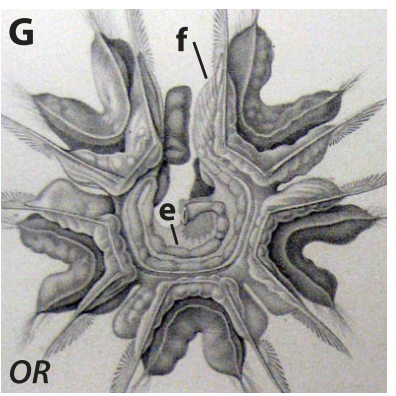

Asthenosoma ijimai (from [35])

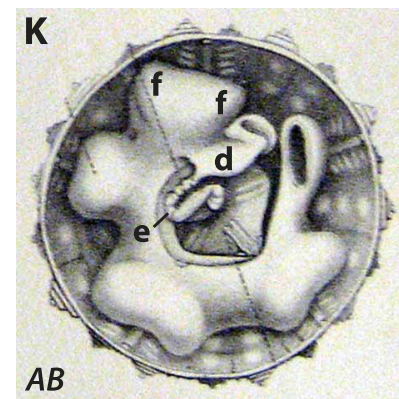

Aspidodiadema meijerei (from [32])

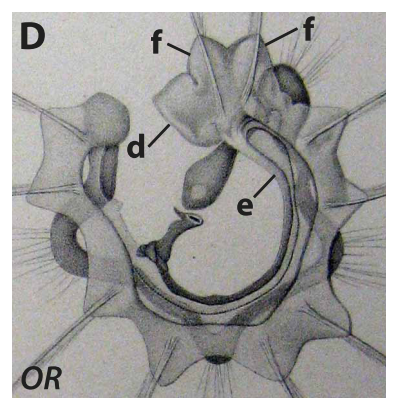

Phormosoma bursarium (from [35])

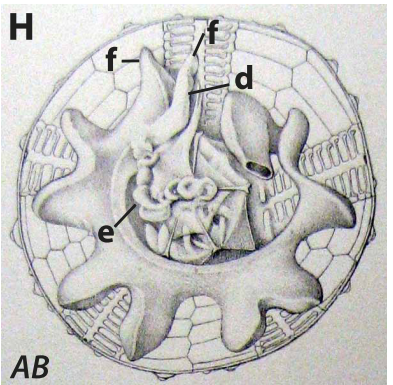

Caenopedina hawaiiensis (from [37])

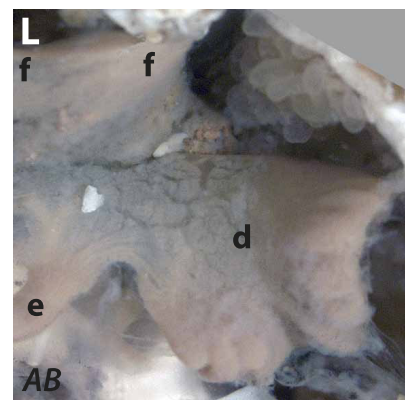

Aspidodiadema hawaiiense (USNM 27590)

Figure 3 Digestive tract anatomy of selected "regular" sea urchin taxa (Histocidaridae - Aspidodiadematidae). Histocidaridae (A), Cidaridae (B, C), Phormosomatidae (D), Echinothuriidae (E-G), Pedinidae ( $H, I)$, Micropygidae $(J)$, and Aspidodiadematidae $(K, L)$. $A B=$ aboral view, $O R=$ oral view. $d=$ dilation, $\mathrm{e}=$ esophagus, $\mathrm{f}=$ festoon. Not to scale.

\section{Cidaridae}

Austrocidaris canaliculata, Cidaris cidaris (Figure 3B, C), Eucidaris metularia (Figure 9A, interactive Figure 10A), Eucidaris thouarsii, Eucidaris tribuloides, Goniocidaris parasol, Hesperocidaris panamensis, Phyllacanthus parvispinus, and Stylocidaris affinis are characterized by an anterior stomach that is located at the border of Amb III and IAmb 3. The esophagus is initially directed towards IAmb 3-Amb III and consists of a short, straight tube. The anterior stomach consists of a single festoon. A small dilation extends adapically just distal to the junction of esophagus and stomach. In his detailed description of the internal anatomy of Cidaris cidaris, Prouho [33] specifically mentioned the absence of any kind of caecum at the junction of esophagus and stomach. Stereocidaris indica deviates from this general description, with a situation that more closely resembles that found in the Histocidaridae and the Ctenocidaridae. Psychocidaridae

The anterior stomach of Psychocidaris ohshimai is located at the border of IAmb 3 and Amb III. The esophagus is initially directed towards Amb III and consists of a short, slightly curved tube. The anterior stomach consists of a single festoon. A small dilation extends adapically and towards IAmb 2 just distal to the junction of esophagus and stomach.

\section{Phormosomatidae}

A long esophagus that is initially directed towards Amb I connects the pharynx to the anterior stomach in Phormosoma bursarium (Figure 3D) and Phormosoma 


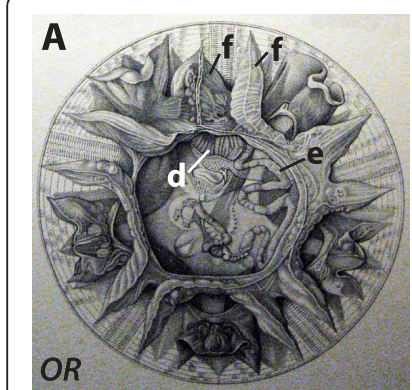

Astropyga radiata

(from [35])

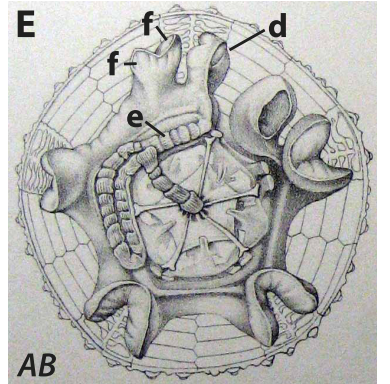

Glyptocidaris crenularis (from [37])

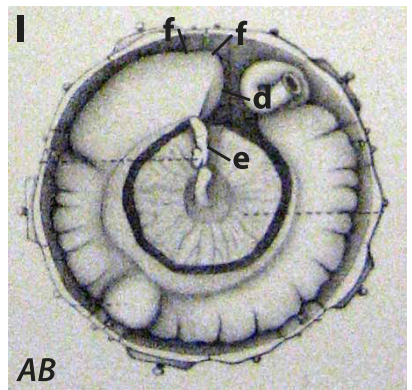

Salenocidaris miliaris (from [32])

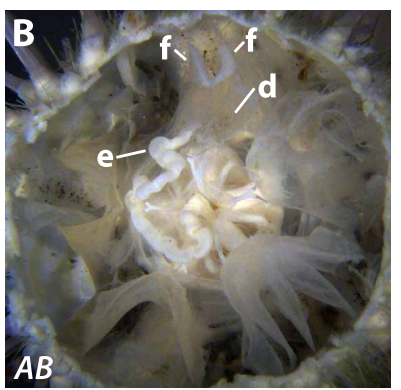

Echinothrix calamaris (this study)

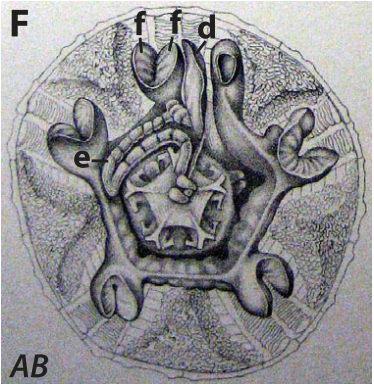

Stomopneustes variolaris (from [37])

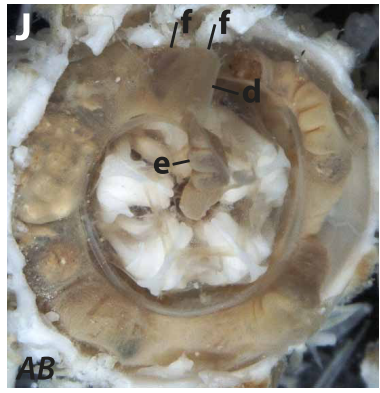

Salenia goesiana

(USNM 10649)

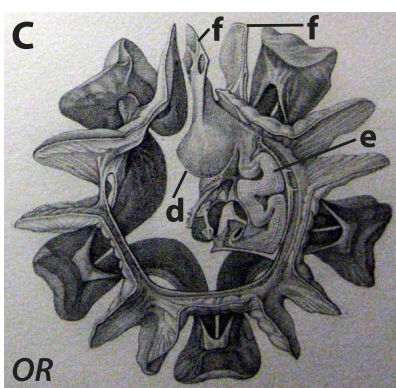

Echinothrix diadema (from [35])

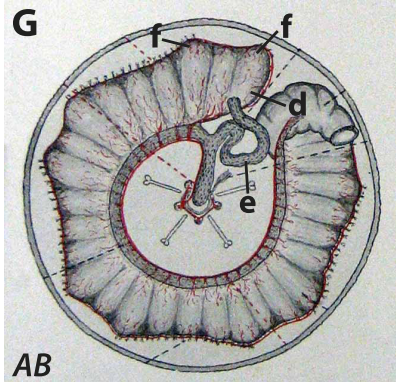

Arbacia lixula (from [22])

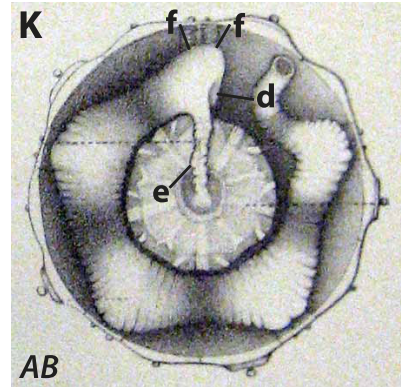

Salenia pattersoni (from [32])

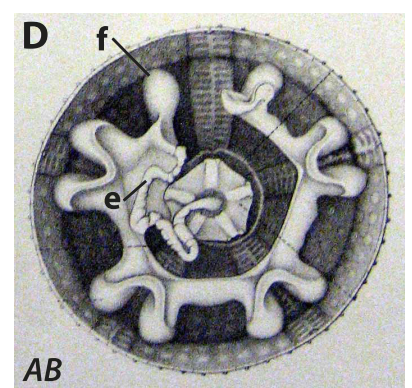

Chaetodiadema pallidum (from [32])

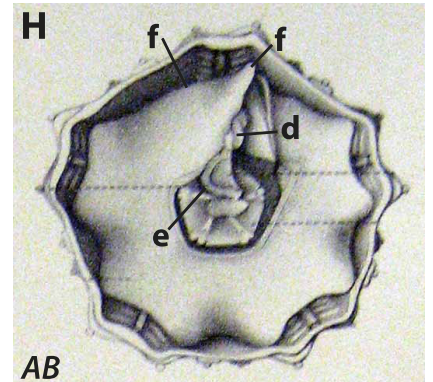

Coelopleurus floridanus (from [32])

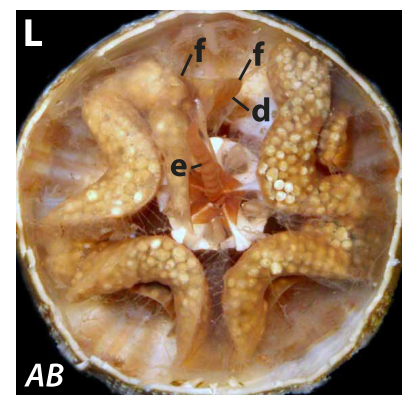

Mespilia globulus (CASIZ 100609)

Figure 4 Digestive tract anatomy of selected "regular" sea urchin taxa (Diadematidae - Temnopleuridae). Diadematidae (A-D), Glyptocidaridae (E), Stomopneustidae (F), Arbaciidae (G, H), Saleniidae (I-K), and Temnopleuridae (L). (G) from [22, Fig. 2, PI. II] - reproduced in modified form with kind permission from L'Institut Océanographique, Fondation Albert ler, Prince de Monaco. $A B=$ aboral view, $L A=$ lateral view, $O R=$ oral view. $d=$ dilation, $e=$ esophagus, $f=$ festoon. Not to scale.

placenta. The anterior stomach spans Amb III and is located between IAmb 3 and IAmb 2. It consists of two separate festoons and a large lateral dilation within IAmb 2. Unfortunately, Schurig [34] did not specifically mention the morphology of the anterior stomach in his detailed report on the internal anatomy of Phormosoma bursarium and a number of other species of the Echinothurioida.

\section{Echinothuriidae}

The digestive tract of the echinothuriid species analyzed so far is characterized by a long esophagus which can sometimes double back on itself. The esophagus in Sperosoma obscurum (Figure 3E) is initially directed towards Amb II but connects with the anterior stomach in Amb
III after making almost a full turn. The anterior stomach of this species consists of two large, separate festoons and a large dilation in IAmb 2. The size of the dilation can vary in echinothuriid species, being largest in Sperosoma obscurum and rather medium-sized in Tromikosoma hispidum (Figure 3F) and Tromikosoma tenue. The anterior stomach of Asthenosoma ijimai (Figure 3G) differs in lacking the dilation as well as the additional festoon of the anterior stomach - however, a conspicuous structure, drawn as part of the intestine, occupies the respective void in Amb III.

\section{Pedinidae}

The species of the genus Caenopedina that have been analyzed so far possess an anterior stomach located 


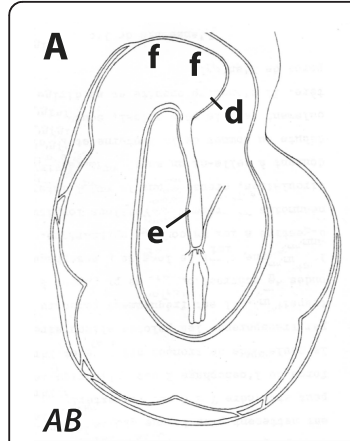

Paracentrotus lividus (from [27])

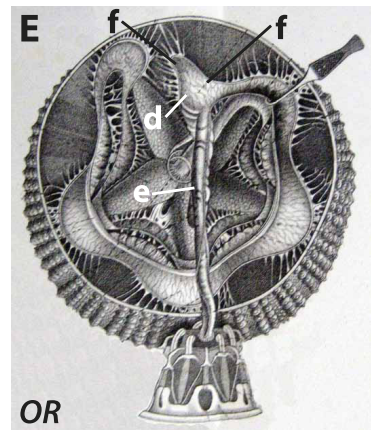

Echinus esculentus (from [21])

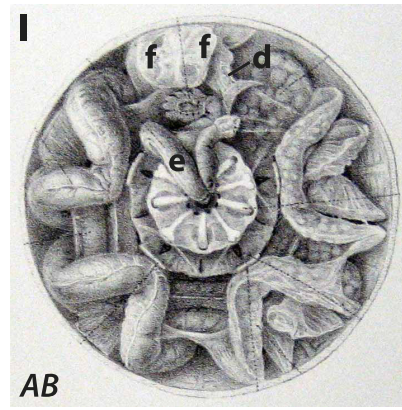

Strongylocentrotus droebachiensis (from [13])

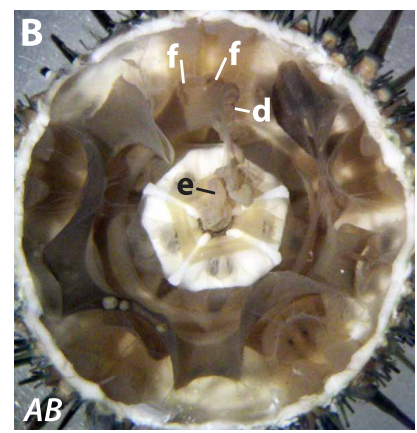

Paracentrotus lividus (this study)

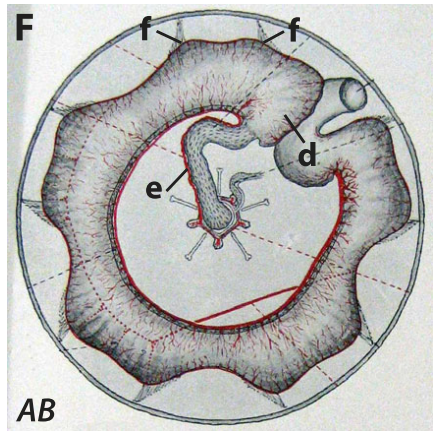

Gracilechinus acutus (from [22])

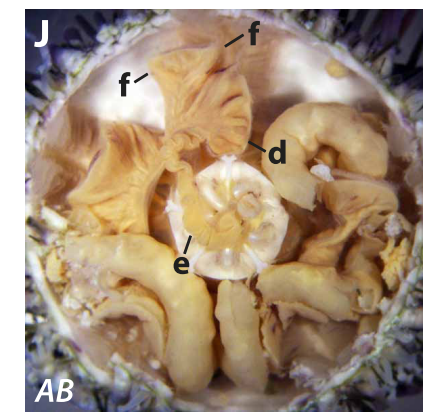

Strongylocentrotus purpuratus (CASIZ 5724)

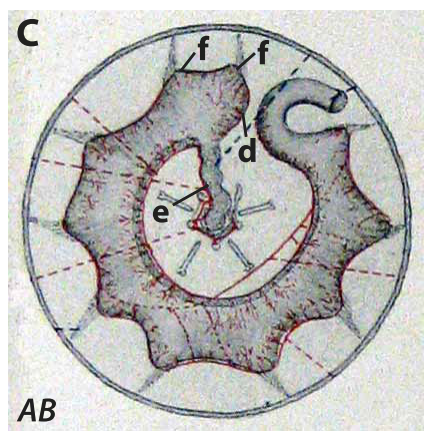

Psammechinus miliaris (from [22])

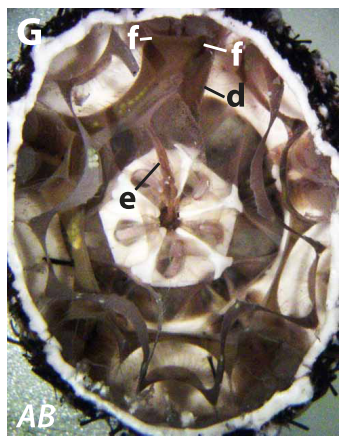

Echinometra mathaei (this study)

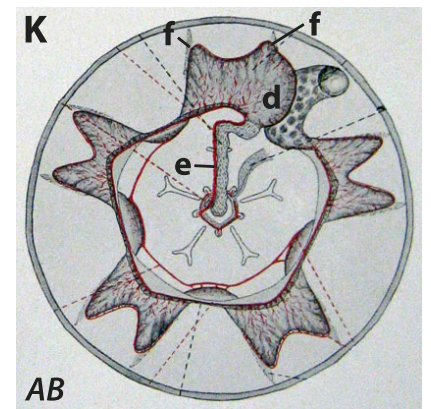

Sphaerechinus granularis (from [22])

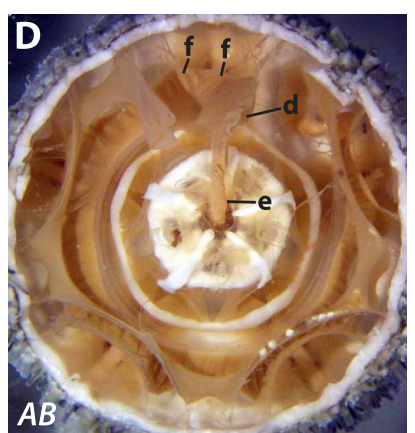

Psammechinus miliaris (this study)

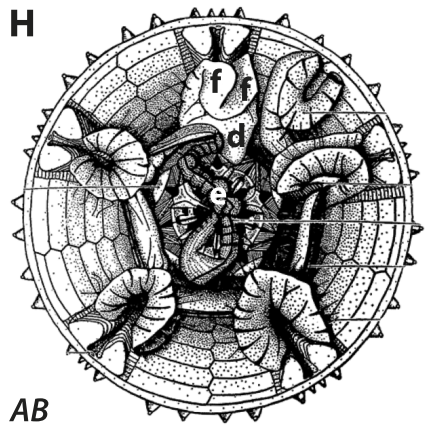

Evechinus chloroticus (from [23])

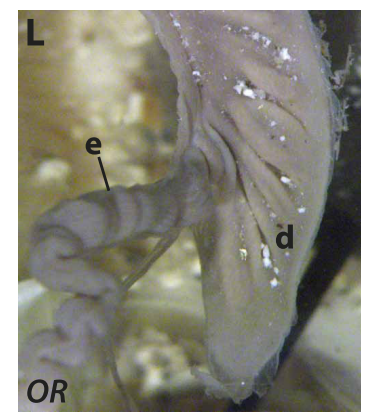

Sphaerechinus granularis (this study)

Figure 5 Digestive tract anatomy of selected "regular" sea urchin taxa (Parechinidae - Toxopneustidae). Parechinidae (A-D), Echinidae (E, F), Echinometridae $(G, H)$, Strongylocentrotidae (I, J), and Toxopneustidae (K, L). (A) from [27, Fig. 9] - reproduced in modified form with kind permission from Mr. Thierry Powis de Tenbossche. (C, F, K) from [22, Figs. 1, 4, 7, PI. II] - reproduced in modified form with kind permission from L'Institut Océanographique, Fondation Albert ler, Prince de Monaco. (H) from [23, Fig. 9] - reproduced in modified form with kind permission from The Royal Society of New Zealand. $A B=$ aboral view, $O R=$ oral view. $\mathrm{d}=$ dilation, $\mathrm{e}=$ esophagus, $\mathrm{f}=$ festoon. Not to scale.

between IAmb 3 and Amb III. The winding esophagus is initially directed towards Amb IV in Caenopedina hawaiiensis (Figure $3 \mathrm{H}$ ) and Caenopedina mirabilis (Figure 3I). The anterior stomach consists of two horizontally fused festoons and a lateral dilation located in Amb III.

\section{Micropygidae}

The anterior stomach of Micropyga tuberculata (Figures 3J, 9B) is located in Amb III, but also reaches laterally into IAmb 2. The winding esophagus is initially directed towards IAmb 5, but connects to the anterior stomach in Amb III. The anterior stomach consists of two separate festoons and a lateral dilation located in IAmb 2. This dilation extends considerably along the oral-aboral axis of the species. Mortensen [38: 142] also noted that "at the passage from the long oesophagus to the intestine there is a large blindsac" (Mortensen's usage of "intestine" = stomach in the present article).

\section{Aspidodiadematidae}

The anterior stomach of Aspidodiadema jacobi, Aspidodiadema meijerei (Figure 3K), Aspidodiadema hawaiiense (Figure 3L), and Plesiodiadema indicum is 


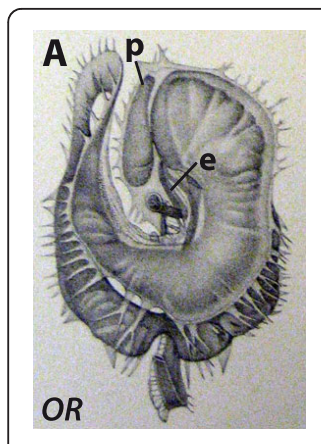

Echinoneus cyclostomus Apatopygus recens (from [50]) (ZMK Mortensen collection)

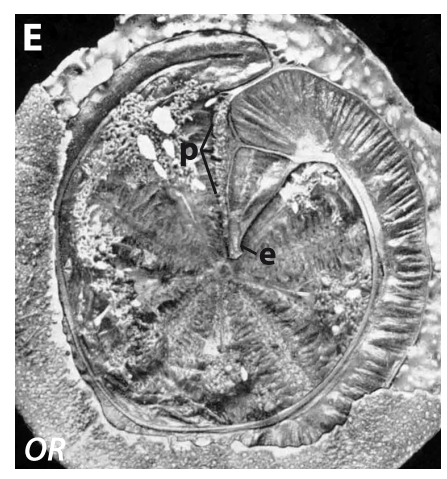

Clypeaster destinatus (from [51])
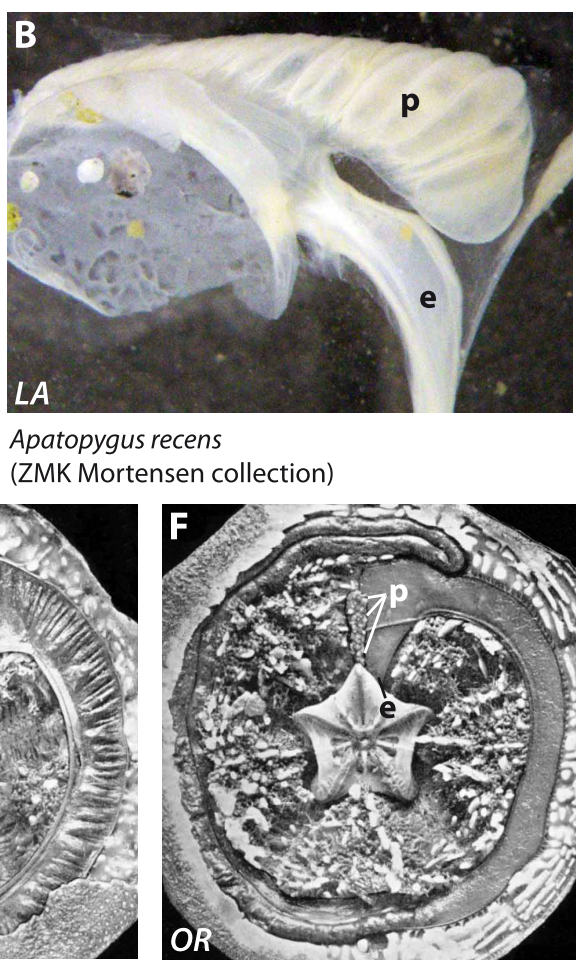

Clypeaster rarispinus (from [51])

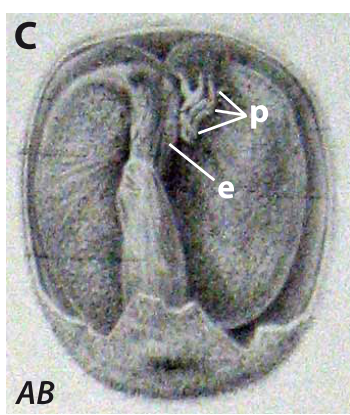

Rhyncholampas pacificus (from [13])

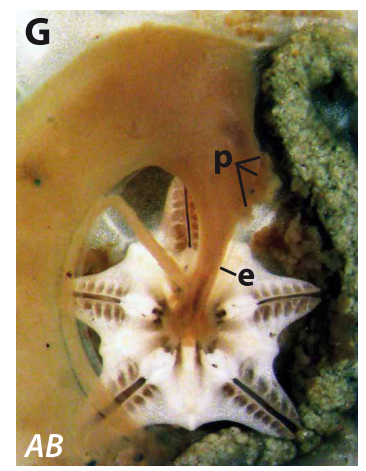

Arachnoides placenta (CASIZ 103170)

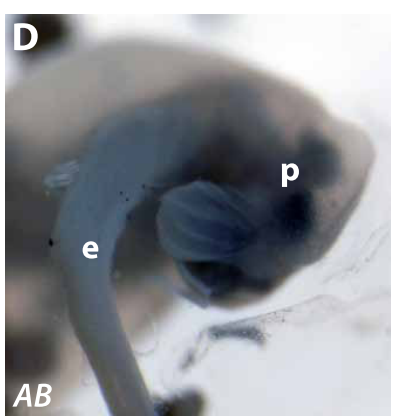

Neolampas rostellata (MNHN EcEh 330)

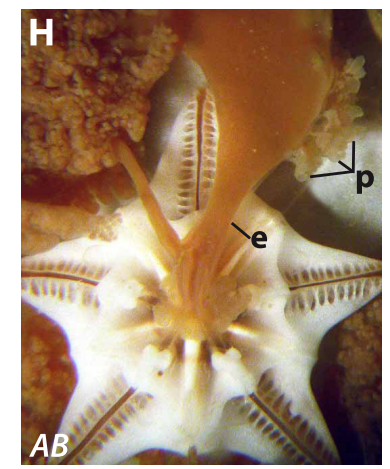

Arachnoides placenta

(CASIZ 94172)

Figure 6 Digestive tract anatomy of selected irregular sea urchin taxa (Echinoneidae - Arachnoididae). Echinoneidae (A), Apatopygidae (B), Cassidulidae (C), Neolampadidae (D), Clypeasteridae (E, F), and Arachnoididae ( $G$ = juvenile specimen, $H=$ adult specimen). $A B=$ aboral view, $L A=$ lateral view,$O R=$ oral view. $\mathrm{e}=$ esophagus, $\mathrm{p}=$ pouch. Not to scale.

located between IAmb 3 and IAmb 2. The winding esophagus is initially directed towards Amb IV before connecting to the anterior stomach in Amb III. The anterior stomach consists of two separate festoons and a conspicuous lateral dilation located in IAmb 2.

\section{Diadematidae}

Diadematids possess a complex anterior stomach that is located between IAmb 2 and IAmb 3. The winding esophagus is initially directed towards Amb IV or V. The anterior part of the stomach in Astropyga radiata (Figure 4A), Diadema antillarum, Diadema savignyi (Figure 9C, interactive Figure 10B), Diadema setosum, Echinothrix calamaris (Figure 4B), and Echinothrix diadema (Figure 4C) consists of two separate festoons and a well-developed dilation. This dilation seems to be less developed in Centrostephanus longispinus and Centrostephanus rodgersii. However, in Chaetodiadema pallidum (Figure 4D), the anterior stomach is characterized by a single festoon only which is located in IAmb 3 . Both the additional festoon and the dilation are absent from this species. According to Lewis [24: 552], the digestive tract of Diadema antillarum consisted "of five sections: esophagus, caecum, foregut, hindgut, and rectum" and "The caecum is a large blind sac. It is continuous with the first loop of the foregut but can be distinguished from the latter by its brighter colour". Lewis also mentioned the presence of "a valve at the junction of caecum and foregut". The above-mentioned dilation can be seen in a recent picture of Diadema setosum (Figure 1A in 28: note the adaxial part of the crenulated structure at the right hand side of the junction of esophagus (es) and stomach (st)). In some diadematid species, particularly in Astropyga, Diadema, and Echinothrix, a fusion of both "ends" of the lower gut loop through mesenteries can be observed at the border of Amb III and IAmb 2 (Figure 4A).

\section{Glyptocidaridae}

The only extant species in this family, Glyptocidaris crenularis (Figure 4E), is characterized by the presence of an anterior stomach that is located in Amb III. The winding esophagus is initially directed towards Amb IV. The anterior stomach consists of two separate festoons and a large dilation that extends slightly into IAmb 2 .

\section{Stomopneustidae}

The digestive tract of Stomopneustes variolaris (Figures $4 \mathrm{~F}, 9 \mathrm{D})$ is very similar to that found in Glyptocidaris crenularis. The anterior stomach consists of two separate festoons. The esophagus displays some degree of 


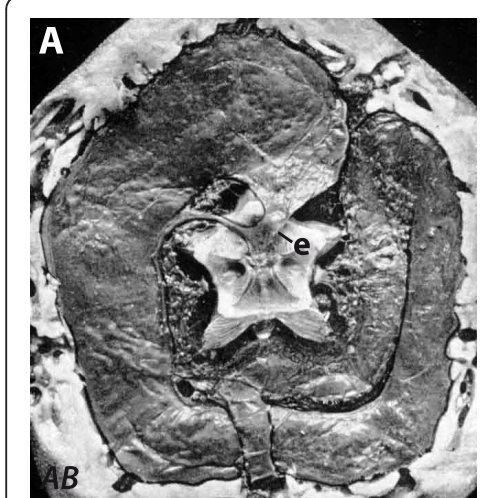

Laganum laganum (from [51])

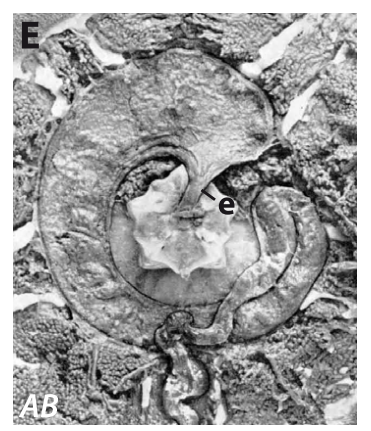

Echinarachnius parma (from [51])

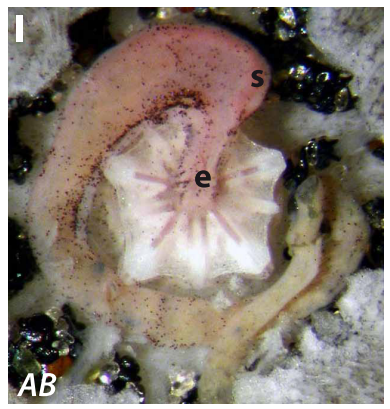

Sinaechinocyamus mai (CASIZ uncataloged material)

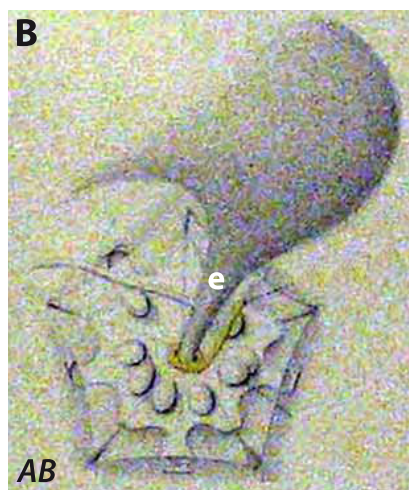

Peronella orbicularis (from [52])

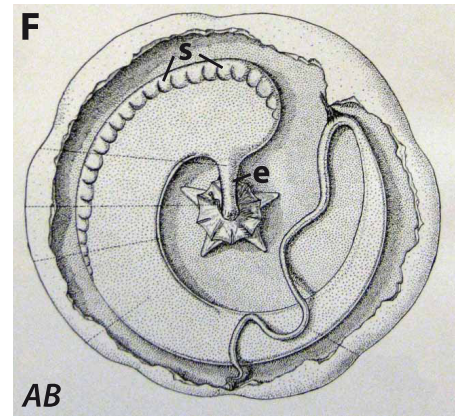

Echinarachnius parma (from [40])

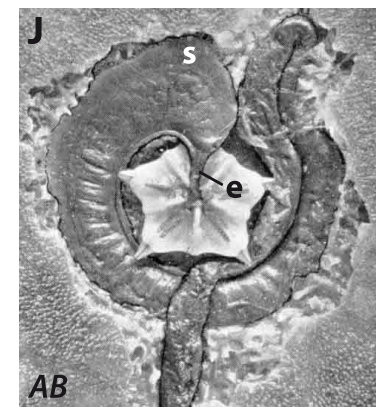

Echinodiscus auritus (from [51])

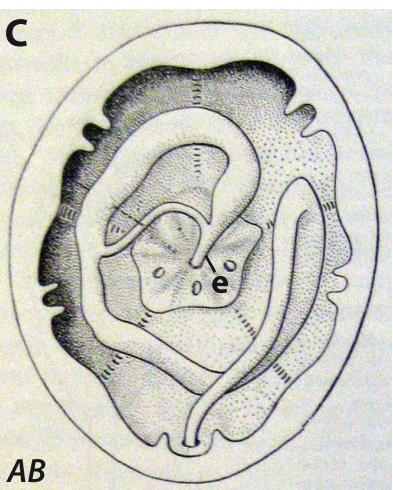

Echinocyamus pusillus (from [52])

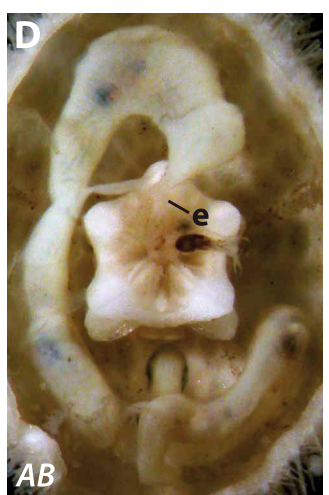

Fibulariella acuta (CASIZ uncataloged mat.)

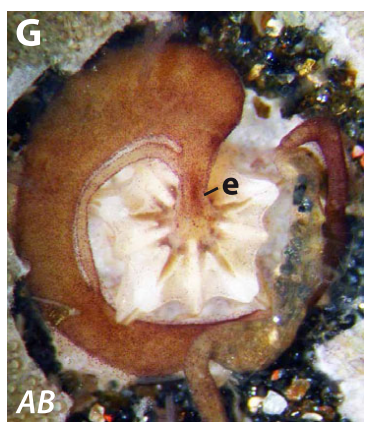

Scaphechinus tenuis (CASIZ 110668)

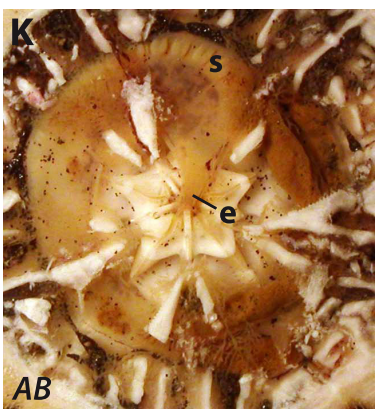

Encope stokesii (CASIZ 3387)

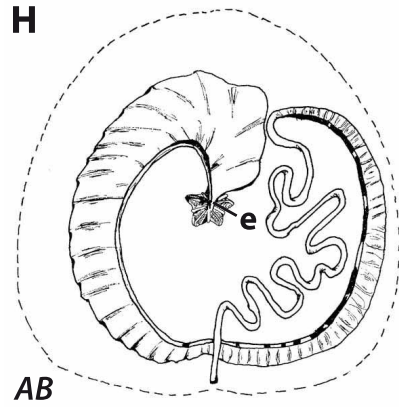

Dendraster excentricus (from [57])
$A B$

Mellita quinquiesperforata (from [40])

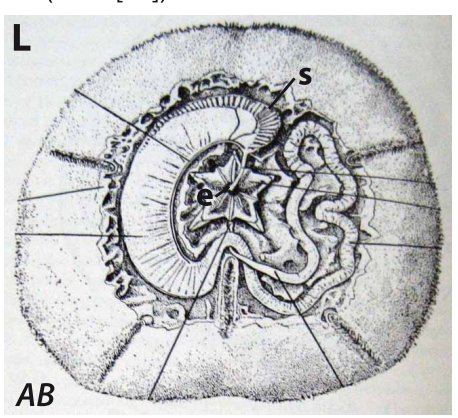

Figure 7 Digestive tract anatomy of selected irregular sea urchin taxa (Laganidae - Mellitidae). Laganidae (A, B), Fibulariidae (C), Rotulidae (D), Echinarachniidae (E, F), Dendrasteridae (G-I), Astriclypeidae (J), and Mellitidae (K, L). (H) from [57, Fig. 3] - reproduced in modified form with kind permission from the Marine Biological Laboratory, Woods Hole, MA, USA. AB = aboral view. $\mathrm{e}=$ esophagus, $\mathrm{s}=$ sacculated abaxial edge of the stomach. Not to scale.

morphological variation and may initially be directed towards Amb III or Amb IV. A large dilation is present at the border of Amb III and IAmb 2.

\section{Arbaciidae}

The arbaciid species analyzed so far, Arbacia dufresnii, Arbacia lixula (Figures 4G, 9E), Arbacia punctulata, and Coelopleurus floridanus (Figure $4 \mathrm{H}$ ), possess an anterior stomach that is located in Amb III. The esophagus is initially directed towards Amb III, but later undulates towards IAmb 2 proximal to its junction with the stomach in Amb III. Due to the horizontally more depressed aspect of the entire arbaciid gut, differentiation of separate festoons and dilations is difficult. However, slight undulations of mesenteries indicate the presence of two horizontally fused festoons in Amb III as well as a small dilation extending into IAmb 2 .

\section{Saleniidae}

Similar to that in arbaciid species, the saleniid gut does not exhibit pronounced vertical festooning. In Salenocidaris miliaris (Figure 4I), Salenia goesiana (Figure 4J), Salenocidaris hastigera (Figure 9F), and Salenia pattersoni (Figure 4K), the short esophagus is initially directed 


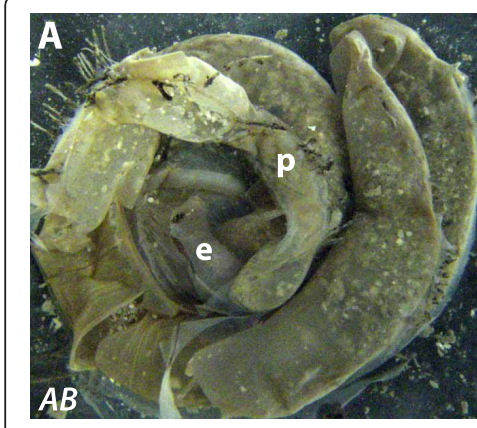

Corystus relictus

(ZMK Mortensen collection)

E

$L A$

Pourtalesia jeffreys

(from [62])

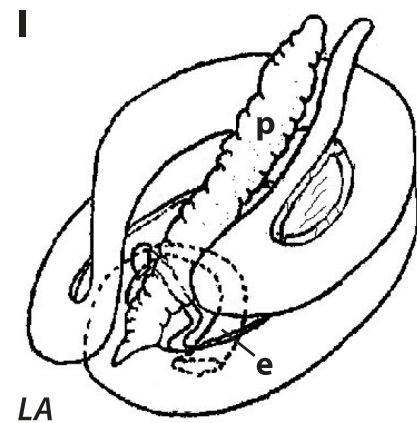

Brisaster latifron

(from Sampson, unpublished data)

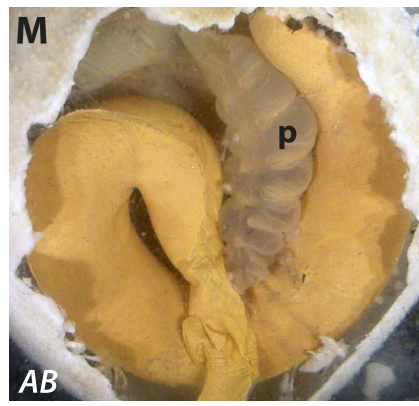

Echinocardium cordatum (this study)

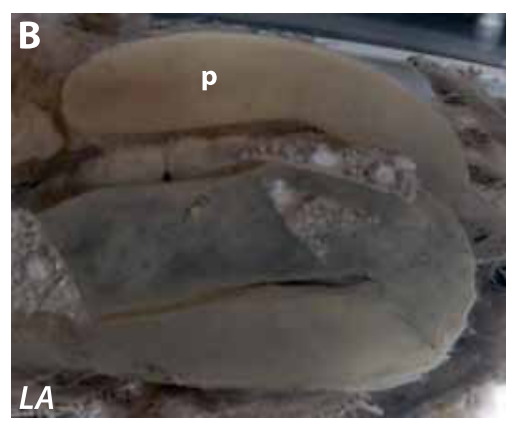

Antrechinus nordenskjoldi (ZMH E7350)

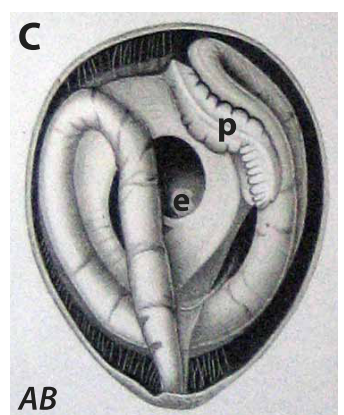

Urechinus naresianus (from [62])

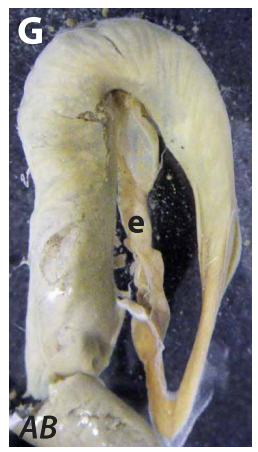

Aeropsis fulva (CASIZ 113902)

Pourtalesia hispida (ZMH E7349)

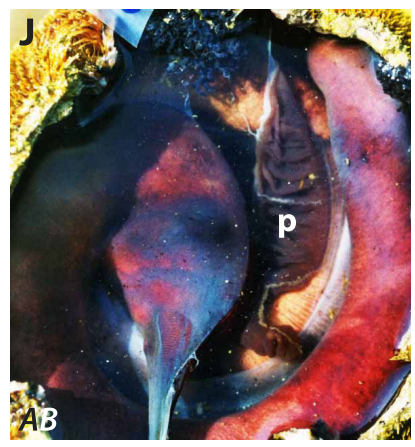

Meoma ventricosa

(this study)

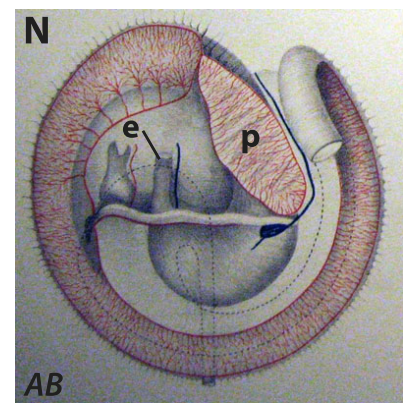

Spatangus purpureus (from [8])

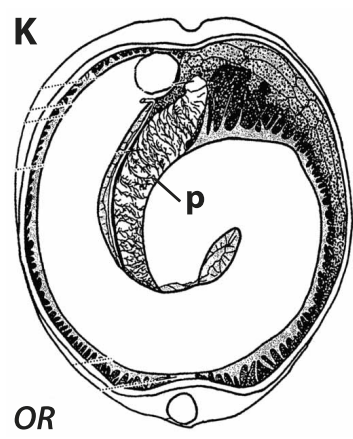

Meoma ventricosa (from [67])

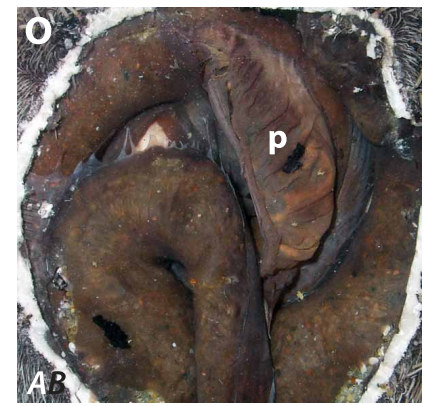

Spatangus purpureus (this study)

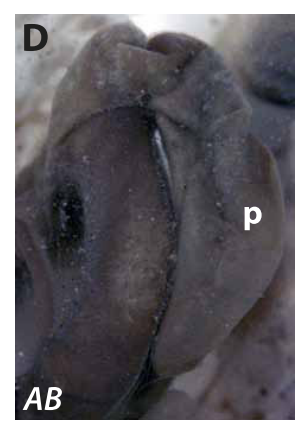

Urechinus naresianus (NHM 1903.8.1.100-4)

Figure 8 Digestive tract anatomy of selected irregular sea urchin taxa (Corystidae - Spatangidae). Corystidae (A), Urechinidae (B-D), Pourtalesiidae (E, F), Aeropsidae (G), Hemiasteridae (H), Schizasteridae (I), Brissidae (J, K), Brissopsidae (L), Loveniidae (M), Spatangidae (N, O), and Asterostomatidae (P). (K) from [67, Fig. 11] - reproduced in modified form with kind permission from the Rosenstiel School of Marine and Atmospheric Science. $A B=$ aboral view, $L A=$ lateral view, $O R=$ oral view. $\mathrm{e}=$ esophagus, $\mathrm{p}=$ pouch. Not to scale. 


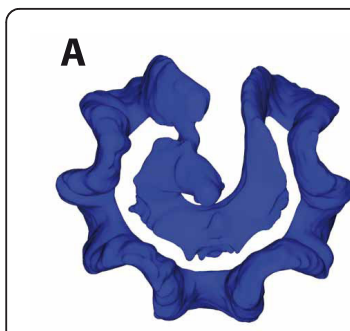

Eucidaris metularia (NHM 1969.5.1.15-40)

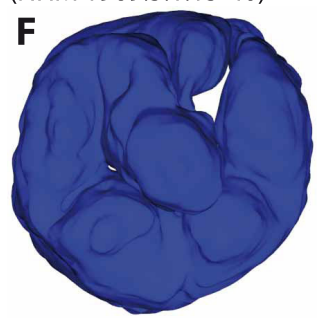

Salenocidaris hastigera (ZMB 5816)

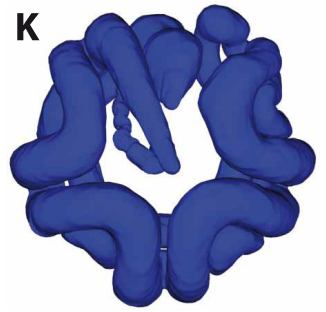

Strongyl. purpuratus (CASIZ 5724)

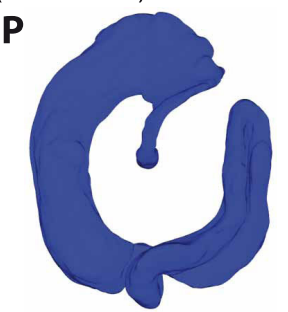

Laganum laganum (CASIZ 94344)

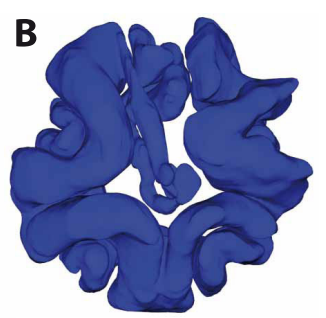

Micropyga tuberculata (NHM 98.8.8.45/6)

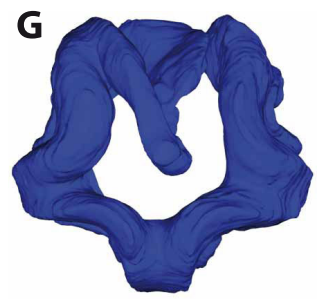

Mespilia globulus (ZMB 5620)

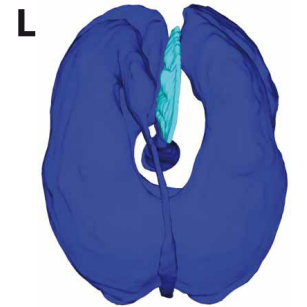

Echinoneus cyclostomus (NHM 1969.5.1.105)

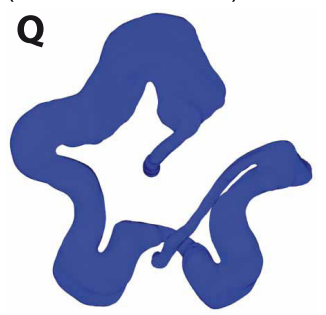

Rotula deciesdigitata (ZMB 2169)

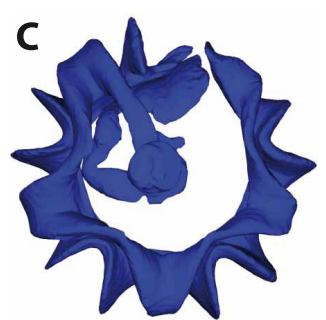

Diadema savignyi (this study)

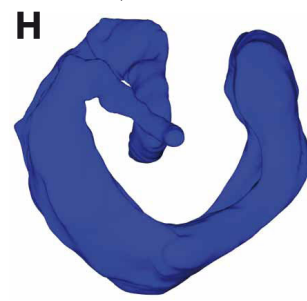

Genocidaris maculata (ZMB 5827)

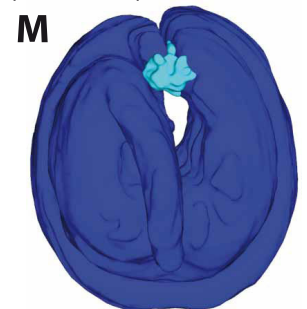

Cassidulus caribaearum (CASIZ 112632)

$\mathbf{R}$

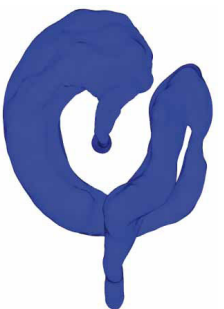

Echinarachnius parma (ZSM 20011676)

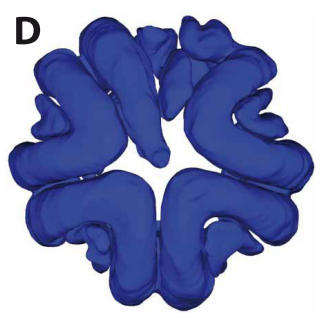

Stomopneustes variolaris (USNM E45930)

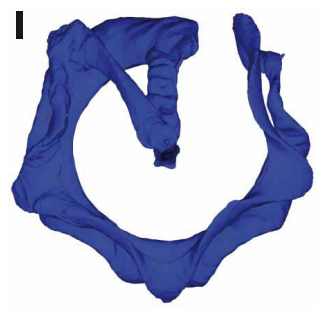

Psammechinus miliaris (this study)

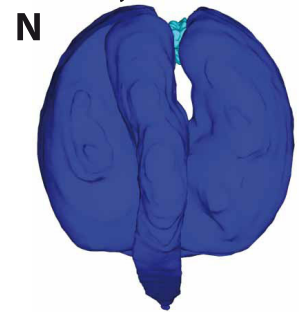

Echinolampas depressa (USNM E32955)

\section{S}

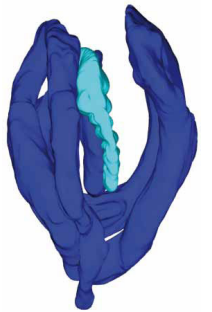

Pourtalesia wandeli (NHM 1976.7.30.76-95)

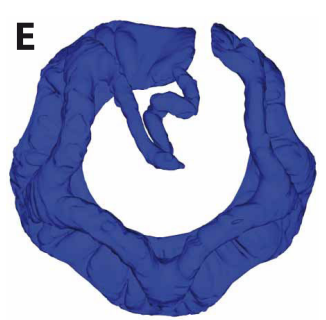

Arbacia lixula (NHM 1952.3.26.31-36)

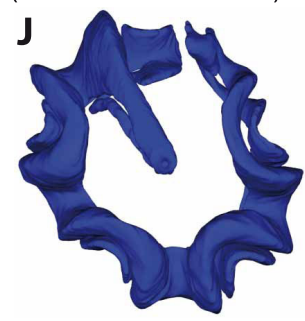

Echinometra mathaei (NHM 1969.5.1.61-75)

O

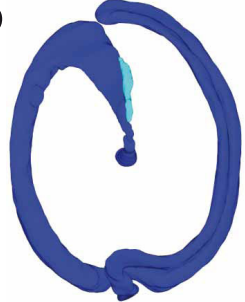

Clypeaster reticulatus (USNM 34282)

$\mathbf{T}$

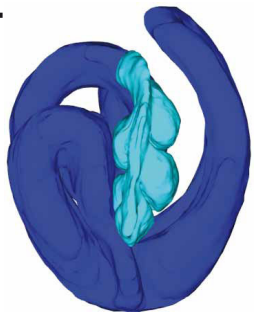

Abatus cavernosus

(ZMB 5854)

Figure 9 Comparative anatomy of the sea urchin digestive tract. (A-T) Aboral views of 3D models that were produced based on magnetic resonance imaging scans of 20 sea urchin species. Cidaridae (A), Micropygidae (B), Diadematidae (C), Stomopneustidae (D), Arbaciidae (E), Saleniidae (F), Temnopleuridae (G), Trigonocidaridae (H), Parechinidae (I), Echinometridae (J), Strongylocentrotidae (K), Echinoneidae (L), Cassidulidae (M), Echinolampadidae (N), Clypeasteridae $(\mathrm{O})$, Laganidae $(\mathrm{P})$, Rotulidae $(\mathrm{Q})$, Echinarachniidae (R), Pourtalesiidae (S), Schizasteridae (T). Dark blue = main digestive tract (comprising the lateral dilation in "regular" euechinoid species (B-K)); cyan = thin-walled pouch(es) in irregular sea urchin species. Not to scale.

towards Amb III where it connects to the anterior stomach. This part of the gut spans between Amb III and IAmb 3 and displays a small lateral dilation directed towards IAmb 2. Slight undulations of mesenteries indicate the presence of two horizontally fused festoons in Amb III.

\section{Temnopleuridae}

Information on the internal anatomy of temnopleurids is available for Amblypneustes pallidus, Holopneustes inflatus, Mespilia globulus (Figures 4L, 9G), Pseudechinus magellanicus, Salmacis bicolor, Temnopleurus michaelseni, and Temnopleurus toreumaticus. MRI analyses indicate that in all species the slightly winding esophagus is initially directed towards Amb III. The anterior stomach is characterized by the presence of two horizontally fused festoons located in Amb III. A small dilation is directed adapically and towards IAmb 2. 

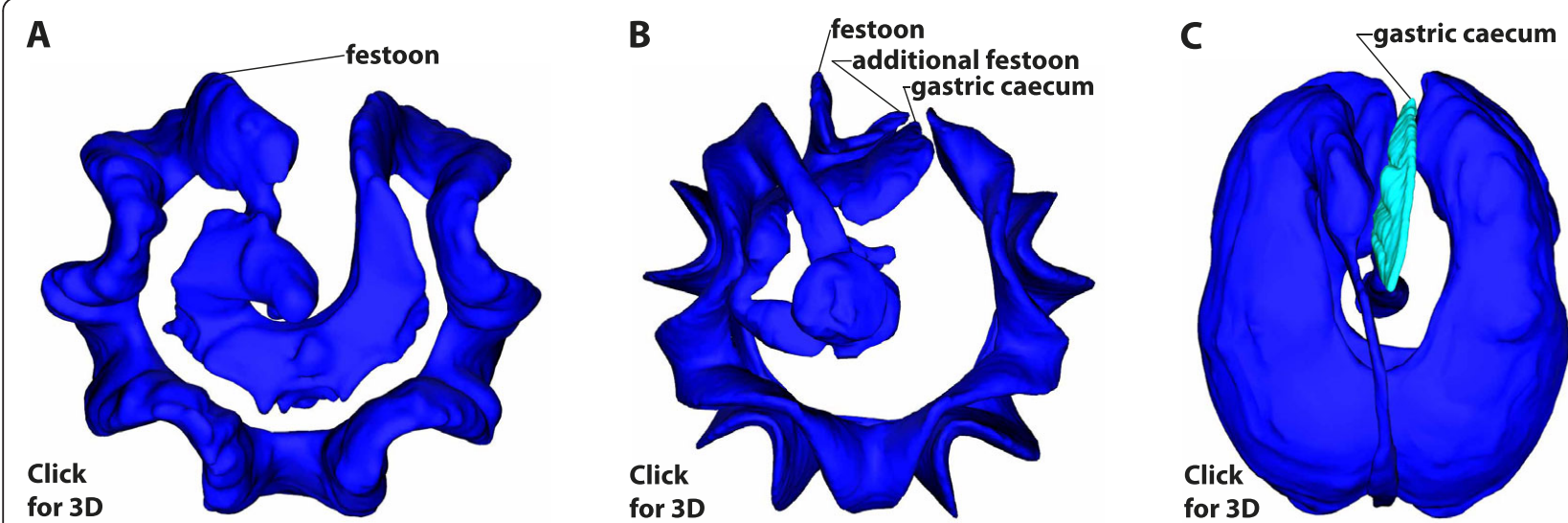

Figure 10 Homology of the sea urchin gastric caecum based on its location as a primary criterion. (A-C) Interactive 3D PDF models of the digestive tract of two "regular" [Eucidaris metularia (A), Diadema savignyi (B)] and one irregular [Echinoneus cyclostomus (C)] sea urchin species. Left-click onto each of the three images in order to activate the embedded 3D models. Labeling designates the structures we consider homologous. Note that the 3D model of Diadema savignyi (B) depicts a modelling artefact due to the close proximity of esophagus and rectum: both structures seem to be fused, although they are clearly not in reality. Please refer to [88-90] for an in-depth explanation of how to manipulate and generate publication-embedded 3D PDF models. This interactive 3D figure requires Adobe Reader 8.0 or higher to operate. Not to scale.

\section{Trigonocidaridae}

MRI scans of Genocidaris maculata (Figure 9H) and Trigonocidaris albida reveal the presence of the anterior stomach in Amb III. The short esophagus is initially directed towards Amb III, where it connects to the stomach. Whereas the anterior stomach in Trigonocidaris albida spans the entire Amb III, also reaching into IAmb 2, the anterior stomach in Genocidaris maculata is located mainly in IAmb 3 and only slightly extends into Amb III. However, both species are characterized by a small dilation of the anterior stomach pointing adapically distal to the junction of esophagus and stomach. Due to the flattened aspect of the entire stomach it is hard to differentiate individual festoons, but slight undulations of mesenteries indicate the presence of two horizontally fused festoons in Amb III.

\section{Parasaleniidae}

All known species in this monogeneric family are characterized by an oval test. In Parasalenia gratiosa, the only species analyzed here, the anterior stomach is largely located in Amb III, but reaches into IAmb 2 and 3 as well. The winding esophagus is initially directed towards IAmb 3, but distally bends towards Amb III where it enters the stomach. The anterior stomach consists of two separate festoons as well as a large dilation located at the border of Amb III and IAmb 2. This dilation extends towards the apex.

\section{Parechinidae}

In specimens of Loxechinus albus, Paracentrotus lividus (Figures 1B, D; 5A, B), Parechinus angulosus, Psammechinus microtuberculatus, and Psammechinus miliaris (Figures 5C, D; 9I), the anterior stomach is located in
Amb III. The short esophagus is initially directed towards Amb III where it also connects to the anterior stomach. The two individual festoons in Amb III are horizontally fused. A small dilation is located just distal to the junction of esophagus and anterior stomach. Several authors have noted this dilation in Paracentrotus lividus, among them Koehler [9], Tiedemann [20], Valentin [21], and Bonnet [22].

\section{Echinidae}

Data on the anterior stomach of echinids are available for eight species: Echinus esculentus (Figure 5E), Echinus melo, Gracilechinus acutus (Figure 5F), Gracilechinus alexandri, Polyechinus agulhensis, Sterechinus agassizi, Sterechinus antarcticus, and Sterechinus neumayeri. In all species, the anterior stomach is largely located in Amb III, but also extends slightly into IAmb 2. The moderately winding esophagus is initially directed towards Amb III, where it also connects to the anterior stomach. This part of the digestive tract is characterized by the presence of two horizontally fused festoons as well as a small dilation that reaches into IAmb 2. According to Koehler [9], this dilation distal to the junction of the esophagus with the anterior stomach is more developed in Echinus melo than in Echinus esculentus.

\section{Echinometridae}

This taxon incorporates species with either an oval or circular test. The anterior stomach in Caenocentrotus gibbosus, Echinometra lucunter, Echinometra mathaei (Figures 5G, 9J), Echinometra viridis, Echinostrephus molaris, Evechinus chloroticus (Figure 5H), Heliocidaris crassispina, Heliocidaris erythrogramma, and Heterocentrotus mammillatus is largely located in Amb III, but 
Table 2 List of "regular"sea urchin taxa included in this study

\begin{tabular}{|c|c|c|c|c|c|}
\hline Order & Family & Species & Method used & Specimen ID & Reference \\
\hline \multirow[t]{16}{*}{ Cidaroida Claus, 1880} & $\begin{array}{l}\text { Histocidaridae Lambert, } \\
1900\end{array}$ & $\begin{array}{l}\text { Histocidaris elegans (Agassiz, } \\
\text { 1879) }\end{array}$ & MRI $(81 \mu \mathrm{m})^{3}$ & ZMH E907 & this study \\
\hline & & $\begin{array}{l}\text { Histocidaris variabilis (Agassiz } \\
\& \text { Clark, 1907) }\end{array}$ & Dissection & - & {$[32]$} \\
\hline & & $\begin{array}{l}\text { Poriocidaris purpurata (Wyville } \\
\text { Thomson, 1872) }\end{array}$ & Dissection & - & this study \\
\hline & $\begin{array}{l}\text { Ctenocidaridae } \\
\text { Mortensen, } 1928\end{array}$ & $\begin{array}{l}\text { Ctenocidaris nutrix (Wyville } \\
\text { Thomson, 1876) }\end{array}$ & MRI $(79 \mu \mathrm{m})^{3}$, dissection & $\begin{array}{l}\text { NHM 1956.10.5.1, AAD } \\
\text { uncataloged material }\end{array}$ & this study \\
\hline & & $\begin{array}{l}\text { Notocidaris gaussensis } \\
\text { Mortensen, } 1909 \\
\end{array}$ & MRI $(79 \mu \mathrm{m})^{3}$ & ZMB 5456 & this study \\
\hline & Cidaridae Gray, 1825 & $\begin{array}{l}\text { Austrocidaris canaliculata } \\
\text { (Agassiz, 1863) }\end{array}$ & MRI $(79 \mu \mathrm{m})^{3}$ & ZMB 2244 & this study \\
\hline & & $\begin{array}{l}\text { Cidaris cidaris (Linnaeus, } \\
1758 \text { ) }\end{array}$ & MRI $(81 \mu \mathrm{m})^{3}$, dissection & $\begin{array}{l}\text { NHM 1925.10.30.103-113, } \\
\text { ZMB } 4803\end{array}$ & $\begin{array}{l}\text { [33], this } \\
\text { study }\end{array}$ \\
\hline & & $\begin{array}{l}\text { Eucidaris metularia (de } \\
\text { Lamarck, 1816) }\end{array}$ & MRI $(81 \mu \mathrm{m})^{3}$ & NHM 1969.5.1.15-40 & this study \\
\hline & & $\begin{array}{l}\text { Eucidaris thouarsii (Agassiz \& } \\
\text { Desor, 1847) }\end{array}$ & MRI $50 \times 50 \times 200 \mu \mathrm{m}^{3}$ & ZMB 1369 & this study \\
\hline & & $\begin{array}{l}\text { Eucidaris tribuloides } \\
\text { Desmoulins, } 1835\end{array}$ & MRI $50 \times 50 \times 200 \mu \mathrm{m}^{3}$ & ZMB 5474 & this study \\
\hline & & Goniocidaris parasol Fell, 1958 & Dissection & NIWA 18974 & this study \\
\hline & & $\begin{array}{l}\text { Hesperocidaris panamensis } \\
\text { (Agassiz, 1898) }\end{array}$ & MRI $50 \times 50 \times 200 \mu \mathrm{m}^{3}$ & ZMB 5407 & this study \\
\hline & & $\begin{array}{l}\text { Phyllacanthus parvispinus } \\
\text { Tenison Woods, } 1880\end{array}$ & Dissection & - & this study \\
\hline & & $\begin{array}{l}\text { Stereocidaris indica Döderlein, } \\
1901\end{array}$ & MRI $(79 \mu \mathrm{m})^{3}$ & ZMB 7364 & this study \\
\hline & & $\begin{array}{l}\text { Stylocidaris affinis (Philippi, } \\
\text { 1845) }\end{array}$ & Dissection & - & this study \\
\hline & $\begin{array}{l}\text { Psychocidaridae Ikeda, } \\
1936\end{array}$ & $\begin{array}{l}\text { Psychocidaris ohshimai lkeda, } \\
1935\end{array}$ & MRI $(79 \mu \mathrm{m})^{3}$ & NHMW 200Z0097/0001 & this study \\
\hline \multirow[t]{6}{*}{$\begin{array}{l}\text { Echinothurioida Claus, } \\
1880\end{array}$} & $\begin{array}{l}\text { Phormosomatidae } \\
\text { Mortensen, } 1934\end{array}$ & $\begin{array}{l}\text { Phormosoma bursarium } \\
\text { Agassiz, } 1881\end{array}$ & Dissection & NIWA 45056, AM J.16209 & $\begin{array}{l}{[34,35] \text {, this }} \\
\text { study }\end{array}$ \\
\hline & & $\begin{array}{l}\text { Phormosoma placenta Wyville } \\
\text { Thomson, } 1872\end{array}$ & Dissection & ZMK Mortensen collection & this study \\
\hline & $\begin{array}{l}\text { Echinothuriidae Wyville } \\
\text { Thomson, } 1872\end{array}$ & $\begin{array}{l}\text { Asthenosoma ijimai } \\
\text { Yoshiwara, } 1897\end{array}$ & Dissection & - & {$[35]$} \\
\hline & & $\begin{array}{l}\text { Sperosoma obscurum Agassiz } \\
\& \text { Clark, } 1907\end{array}$ & Dissection & - & {$[35]$} \\
\hline & & $\begin{array}{l}\text { Tromikosoma hispidum } \\
\text { (Agassiz, 1898) }\end{array}$ & Dissection & - & {$[35]$} \\
\hline & & $\begin{array}{l}\text { Tromikosoma tenue (Agassiz, } \\
\text { 1879) }\end{array}$ & Dissection & - & {$[36]$} \\
\hline \multirow[t]{2}{*}{$\begin{array}{l}\text { Pedinoida Mortensen, } \\
1939\end{array}$} & Pedinidae Pomel, 1883 & $\begin{array}{l}\text { Caenopedina hawaiiensis } \\
\text { Agassiz \& Clark, } 1907\end{array}$ & Dissection & - & {$[37]$} \\
\hline & & $\begin{array}{l}\text { Caenopedina mirabilis } \\
\text { (Döderlein, 1885) }\end{array}$ & MRI $(81 \mu \mathrm{m})^{3}$, dissection & $\begin{array}{l}\text { USNM 31178, USNM } \\
31182, \text { AM J.24188 }\end{array}$ & this study \\
\hline \multirow[t]{4}{*}{$\begin{array}{l}\text { Diadematoida } \\
\text { Duncan, } 1889 \\
\end{array}$} & $\begin{array}{l}\text { Micropygidae } \\
\text { Mortensen, } 1903\end{array}$ & $\begin{array}{l}\text { Micropyga tuberculata } \\
\text { Agassiz, } 1879\end{array}$ & MRI $(81 \mu \mathrm{m})^{3}$, dissection & $\begin{array}{l}\text { NHM 98.8.8.45/6, ZMK } \\
\text { Mortensen collection }\end{array}$ & $\begin{array}{l}{[35,38] \text {, this }} \\
\text { study }\end{array}$ \\
\hline & $\begin{array}{l}\text { Aspidodiadematidae } \\
\text { Duncan, } 1889\end{array}$ & $\begin{array}{l}\text { Aspidodiadema hawaiiense } \\
\text { Mortensen, } 1939\end{array}$ & MRI $(81 \mu \mathrm{m})^{3}$, dissection & USNM 27590 & this study \\
\hline & & $\begin{array}{l}\text { Aspidodiadema jacobi Agassiz, } \\
1880\end{array}$ & Dissection & - & {$[39]$} \\
\hline & & $\begin{array}{l}\text { Aspidodiadema meijerei } \\
\text { (Döderlein, 1906) }\end{array}$ & Dissection & - & {$[32]$} \\
\hline
\end{tabular}


Table 2 List of "regular"sea urchin taxa included in this study (Continued)

\begin{tabular}{|c|c|c|c|c|c|}
\hline & & $\begin{array}{l}\text { Plesiodiadema indicum } \\
\text { (Döderlein, 1900) }\end{array}$ & MRI $(81 \mu \mathrm{m})^{3}$ & ZMB 7232 & this study \\
\hline & $\begin{array}{l}\text { Diadematidae Gray, } \\
1855\end{array}$ & $\begin{array}{l}\text { Astropyga radiata (Leske, } \\
\text { 1778) }\end{array}$ & Dissection & - & {$[35]$} \\
\hline & & $\begin{array}{l}\text { Centrostephanus longispinus } \\
\text { (Philippi, 1845) }\end{array}$ & MRI $(66 \mu \mathrm{m})^{3}$ & NHM 1952.3.26.64-8 & this study \\
\hline & & $\begin{array}{l}\text { Centrostephanus rodgersii } \\
\text { (Agassiz, 1863) }\end{array}$ & Dissection & - & this study \\
\hline & & $\begin{array}{l}\text { Chaetodiadema pallidum } \\
\text { (Agassiz \& Clark, 1907) }\end{array}$ & Dissection & - & {$[32]$} \\
\hline & & $\begin{array}{l}\text { Diadema antillarum Philippi, } \\
1845\end{array}$ & $\begin{array}{l}\text { MRI } 50 \times 50 \times 200 \\
\mu^{3} \text {, dissection }\end{array}$ & ZMB 4374 & $\begin{array}{l}\text { [24], this } \\
\text { study }\end{array}$ \\
\hline & & $\begin{array}{l}\text { Diadema savignyi Michelin, } \\
1845\end{array}$ & MRI $(40 \mu \mathrm{m})^{3}$ & - & this study \\
\hline & & $\begin{array}{l}\text { Diadema setosum (Leske, } \\
\text { 1778) }\end{array}$ & Dissection & - & $\begin{array}{l}{[13,28], \text { this }} \\
\text { study }\end{array}$ \\
\hline & & $\begin{array}{l}\text { Echinothrix calamaris (Pallas, } \\
\text { 1774) }\end{array}$ & Dissection & - & this study \\
\hline & & $\begin{array}{l}\text { Echinothrix diadema } \\
\text { (Linnaeus, 1758) }\end{array}$ & $\begin{array}{l}\text { MRI } 50 \times 50 \times 200 \\
{\mu m^{3}}^{3} \text { dissection }\end{array}$ & ZMB 2346 & $\begin{array}{l}\text { [35], this } \\
\text { study }\end{array}$ \\
\hline Incerta sedis & $\begin{array}{l}\text { Glyptocidaridae Jensen, } \\
1982\end{array}$ & $\begin{array}{l}\text { Glyptocidaris crenularis } \\
\text { Agassiz, } 1864\end{array}$ & Dissection & - & {$[37]$} \\
\hline Incerta sedis & $\begin{array}{l}\text { Stomopneustidae } \\
\text { Mortensen, } 1903\end{array}$ & $\begin{array}{l}\text { Stomopneustes variolaris (de } \\
\text { Lamarck, 1816) }\end{array}$ & MRI $(81 \mu \mathrm{m})^{3}$, dissection & USNM E45930 & $\begin{array}{l}\text { [37], this } \\
\text { study }\end{array}$ \\
\hline \multirow[t]{4}{*}{$\begin{array}{l}\text { Arbacioida Gregory, } \\
1900\end{array}$} & Arbaciidae Gray, 1855 & $\begin{array}{l}\text { Arbacia dufresnii (de Blainville, } \\
\text { 1825) }\end{array}$ & MRI $50 \times 50 \times 200 \mu \mathrm{m}^{3}$ & ZMB 2222 & this study \\
\hline & & Arbacia lixula (Linnaeus, 1758) & MRI $(81 \mu \mathrm{m})^{3}$, dissection & $\begin{array}{l}\text { NHM 1952.3.26.31-36, ZMB } \\
7203\end{array}$ & $\begin{array}{l}\text { [22], this } \\
\text { study }\end{array}$ \\
\hline & & $\begin{array}{l}\text { Arbacia punctulata (de } \\
\text { Lamarck, 1816) }\end{array}$ & Dissection & - & [40] \\
\hline & & $\begin{array}{l}\text { Coelopleurus floridanus } \\
\text { Agassiz, } 1871\end{array}$ & Dissection & - & {$[32]$} \\
\hline \multirow[t]{4}{*}{$\begin{array}{l}\text { Salenioida Delage \& } \\
\text { Herouard, } 1903\end{array}$} & Saleniidae Agassiz, 1838 & Salenia goesiana Lovén, 1874 & Dissection & USNM 10649 & this study \\
\hline & & $\begin{array}{l}\text { Salenia pattersoni Agassiz, } \\
1878\end{array}$ & Dissection & - & [32] \\
\hline & & $\begin{array}{l}\text { Salenocidaris hastigera } \\
\text { Agassiz, } 1869\end{array}$ & MRI $(81 \mu \mathrm{m})^{3}$ & ZMB 5816 & this study \\
\hline & & $\begin{array}{l}\text { Salenocidaris miliaris Agassiz, } \\
1869\end{array}$ & Dissection & - & [32] \\
\hline \multirow[t]{8}{*}{$\begin{array}{l}\text { Temnopleuroida } \\
\text { Mortensen, } 1942\end{array}$} & $\begin{array}{l}\text { Temnopleuridae } \\
\text { Agassiz, } 1872\end{array}$ & $\begin{array}{l}\text { Amblypneustes pallidus (de } \\
\text { Lamarck, 1816) }\end{array}$ & MRI $50 \times 50 \times 200 \mu^{3}$ & ZMB 6334 & this study \\
\hline & & $\begin{array}{l}\text { Holopneustes inflatus Lutken, } \\
1872\end{array}$ & MRI $50 \times 50 \times 200 \mu^{3}$ & ZMB 2639 & this study \\
\hline & & $\begin{array}{l}\text { Mespilia globulus (Linnaeus, } \\
\text { 1758) }\end{array}$ & MRI $(44 \mu \mathrm{m})^{3}$ & ZMB 5620, CASIZ 100609 & this study \\
\hline & & $\begin{array}{l}\text { Pseudechinus magellanicus } \\
\text { Philippi, } 1857\end{array}$ & MRI $50 \times 50 \times 200 \mu \mathrm{m}^{3}$ & ZMB 2188 & this study \\
\hline & & $\begin{array}{l}\text { Salmacis bicolor (Agassiz, } \\
\text { 1846) }\end{array}$ & Dissection & - & {$[41]$} \\
\hline & & $\begin{array}{l}\text { Temnopleurus michaelseni } \\
\text { (Döderlein, 1914) }\end{array}$ & MRI $50 \times 50 \times 200 \mu \mathrm{m}^{3}$ & ZMB 6331 & this study \\
\hline & & $\begin{array}{l}\text { Temnopleurus toreumaticus } \\
\text { (Leske, 1778) }\end{array}$ & $\begin{array}{l}\mathrm{MRI} 78 \times 78 \times 300 \\
\mathrm{~mm}^{3}, \text { dissection }\end{array}$ & ZMB 5511, ZMB 2802 & this study \\
\hline & $\begin{array}{l}\text { Trigonocidaridae } \\
\text { Mortensen, } 1903\end{array}$ & $\begin{array}{l}\text { Genocidaris maculata Agassiz, } \\
1869\end{array}$ & MRI $(36 \mu \mathrm{m})^{3}$ & ZMB 5827 & this study \\
\hline
\end{tabular}


Table 2 List of "regular"sea urchin taxa included in this study (Continued)

\begin{tabular}{|c|c|c|c|c|c|}
\hline & & $\begin{array}{l}\text { Trigonocidaris albida Agassiz, } \\
1869\end{array}$ & MRI $(32 \mu \mathrm{m})^{3}$ & ZSM 20012468 & this study \\
\hline \multirow[t]{27}{*}{$\begin{array}{l}\text { Echinoida Troschel, } \\
1872\end{array}$} & $\begin{array}{l}\text { Parasaleniidae } \\
\text { Mortensen, } 1940\end{array}$ & $\begin{array}{l}\text { Parasalenia gratiosa Agassiz, } \\
1864\end{array}$ & MRI $(79 \mu \mathrm{m})^{3}$ & NHM 1983.2.15.7 & this study \\
\hline & $\begin{array}{l}\text { Parechinidae } \\
\text { Mortensen, } 1903 \\
\end{array}$ & $\begin{array}{l}\text { Loxechinus albus (Molina, } \\
\text { 1782) }\end{array}$ & MRI $50 \times 50 \times 200 \mu \mathrm{m}^{3}$ & NHM 1966.9.27.35 & this study \\
\hline & & $\begin{array}{l}\text { Paracentrotus lividus (de } \\
\text { Lamarck, 1816) }\end{array}$ & MRI $(81 \mu \mathrm{m})^{3}$, dissection & - & $\begin{array}{l}{[20-22,27]} \\
\text { this study }\end{array}$ \\
\hline & & $\begin{array}{l}\text { Parechinus angulosus (Leske, } \\
\text { 1778) }\end{array}$ & MRI $50 \times 50 \times 200 \mu^{3}$ & ZMB 5644 & this study \\
\hline & & $\begin{array}{l}\text { Psammechinus } \\
\text { microtuberculatus (de } \\
\text { Blainville, 1825) }\end{array}$ & $\begin{array}{l}\text { MRI } 50 \times 50 \times 200 \\
\mu^{3} \text {, dissection }\end{array}$ & ZMB 4770 & $\begin{array}{l}{[22] \text {, this }} \\
\text { study }\end{array}$ \\
\hline & & $\begin{array}{l}\text { Psammechinus miliaris } \\
\text { (Müller, 1771) }\end{array}$ & MRI $(44 \mu \mathrm{m})^{3}$, dissection & - & $\begin{array}{l}{[22,42], \text { this }} \\
\text { study }\end{array}$ \\
\hline & Echinidae Gray, 1825 & $\begin{array}{l}\text { Echinus esculentus Linnaeus, } \\
1758\end{array}$ & MRI $(81 \mu \mathrm{m})^{3}$, dissection & ZMB 3826 & $\begin{array}{l}{[21,43-46]} \\
\text { this study }\end{array}$ \\
\hline & & $\begin{array}{l}\text { Echinus melo de Lamarck, } \\
1816\end{array}$ & Dissection & - & [9] \\
\hline & & $\begin{array}{l}\text { Gracilechinus acutus (de } \\
\text { Lamarck, 1816) }\end{array}$ & $\begin{array}{l}\mathrm{MRI} 78 \times 78 \times 300 \\
\mu^{3} \text {, dissection }\end{array}$ & ZMB 3604 & $\begin{array}{l}{[22], \text { this }} \\
\text { study }\end{array}$ \\
\hline & & $\begin{array}{l}\text { Gracilechinus alexandri } \\
\text { (Danielssen \& Koren, 1883) }\end{array}$ & MRI $50 \times 50 \times 200 \mu \mathrm{m}^{3}$ & ZMB 4340 & this study \\
\hline & & $\begin{array}{l}\text { Polyechinus agulhensis } \\
\text { (Döderlein, 1905) }\end{array}$ & MRI $50 \times 50 \times 200 \mu \mathrm{m}^{3}$ & ZMB 7219 & this study \\
\hline & & $\begin{array}{l}\text { Sterechinus agassizi } \\
\text { Mortensen, } 1910\end{array}$ & MRI $(79 \mu \mathrm{m})^{3}$ & NHM 1914.8.12.126-127 & this study \\
\hline & & $\begin{array}{l}\text { Sterechinus antarcticus } \\
\text { Koehler, } 1901\end{array}$ & MRI $50 \times 50 \times 200 \mu \mathrm{m}^{3}$ & ZMB 5439 & this study \\
\hline & & $\begin{array}{l}\text { Sterechinus neumayeri } \\
\text { (Meissner, 1900) }\end{array}$ & MRI $50 \times 50 \times 200 \mu \mathrm{m}^{3}$ & ZMB uncataloged material & this study \\
\hline & $\begin{array}{l}\text { Echinometridae Gray, } \\
1855\end{array}$ & $\begin{array}{l}\text { Caenocentrotus gibbosus } \\
\text { (Agassiz \& Desor, 1840) }\end{array}$ & MRI $50 \times 50 \times 200 \mu^{3}$ & ZMB 5405 & this study \\
\hline & & $\begin{array}{l}\text { Echinometra lucunter } \\
\text { (Linnaeus, 1758) }\end{array}$ & Dissection & ZMB 5511 & this study \\
\hline & & $\begin{array}{l}\text { Echinometra mathaei (de } \\
\text { Blainville, 1825) }\end{array}$ & MRI $(81 \mu \mathrm{m})^{3}$ & NHM 1969.5.1.61-75 & this study \\
\hline & & $\begin{array}{l}\text { Echinometra viridis Agassiz, } \\
1863\end{array}$ & $\begin{array}{l}\text { MRI } 50 \times 50 \times 200 \\
\mu^{3} \text {, dissection }\end{array}$ & ZMB 1827, ZMB 5503 & this study \\
\hline & & $\begin{array}{l}\text { Echinostrephus molaris (de } \\
\text { Blainville, 1825) }\end{array}$ & MRI $50 \times 50 \times 200 \mu \mathrm{m}^{3}$ & ZMB 4000 & this study \\
\hline & & $\begin{array}{l}\text { Evechinus chloroticus } \\
\text { (Valenciennes, 1846) }\end{array}$ & Dissection & - & {$[23]$} \\
\hline & & $\begin{array}{l}\text { Heliocidaris crassispina } \\
\text { (Agassiz, 1863) }\end{array}$ & MRI $50 \times 50 \times 200 \mu \mathrm{m}^{3}$ & ZMB 6424 & this study \\
\hline & & $\begin{array}{l}\text { Heliocidaris erythrogramma } \\
\text { (Valenciennes, 1846) }\end{array}$ & MRI $50 \times 50 \times 200 \mu \mathrm{m}^{3}$ & ZMB 5745 & this study \\
\hline & & $\begin{array}{l}\text { Heterocentrotus mammillatus } \\
\text { (Linnaeus, 1758) }\end{array}$ & MRI $50 \times 50 \times 200 \mu \mathrm{m}^{3}$ & ZMB 1567 & this study \\
\hline & $\begin{array}{l}\text { Strongylocentrotidae } \\
\text { Gregory, } 1900\end{array}$ & $\begin{array}{l}\text { Pseudocentrotus depressus } \\
\text { (Agassiz, 1863) }\end{array}$ & MRI $50 \times 50 \times 200 \mu \mathrm{m}^{3}$ & ZMB 6426 & this study \\
\hline & & $\begin{array}{l}\text { Strongylocentrotus } \\
\text { droebachiensis (Müller, 1776) }\end{array}$ & $\begin{array}{l}\text { MRI } 50 \times 50 \times 200 \\
\mu^{3} \text {, dissection }\end{array}$ & ZMB 4446, ZMB 4422 & $\begin{array}{l}{[13,47] \text {, this }} \\
\text { study }\end{array}$ \\
\hline & & $\begin{array}{l}\text { Strongylocentrotus purpuratus } \\
\text { (Stimpson, 1857) }\end{array}$ & MRI $(44 \mu \mathrm{m})^{3}$, dissection & CASIZ 5724 & $\begin{array}{l}\text { [26], this } \\
\text { study }\end{array}$ \\
\hline & $\begin{array}{l}\text { Toxopneustidae } \\
\text { Troschel, } 1872\end{array}$ & $\begin{array}{l}\text { Gymnechinus robillardi (de } \\
\text { Loriol, 1883) }\end{array}$ & MRI $(79 \mu \mathrm{m})^{3}$ & NHM 1890.6.27.5-8 & this study \\
\hline
\end{tabular}


Table 2 List of "regular"sea urchin taxa included in this study (Continued)

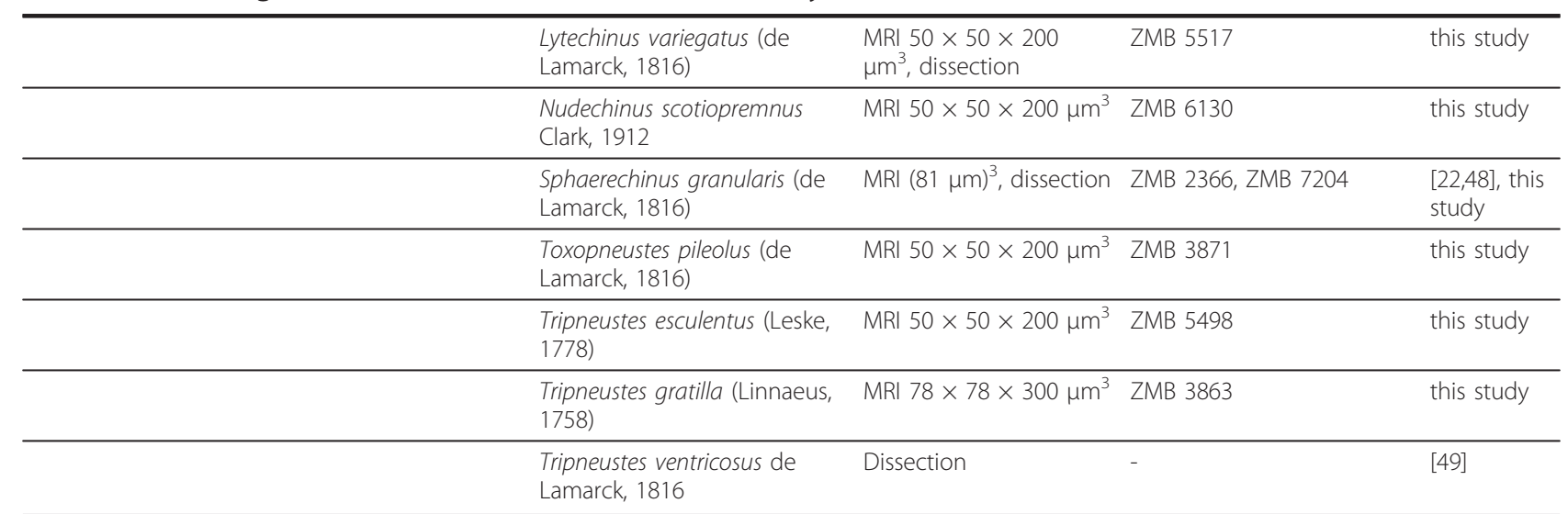

The table provides information on every species studied so far with regard to digestive tract anatomy, listing the method(s) used, the specimen ID of museum specimens where applicable, and the respective references. Numbers in brackets behind "MRI" represent the resolution of the dataset. An overview of scanning parameters is provided by $[25,86]$. this study = specimens were dissected and/or scanned in the course of this study; see the 'List of abbreviations used' section for an explanation of abbreviations.

extends into IAmb 2 and 3 as well. The winding esophagus is initially directed towards the apex before descending towards IAmb 3; it joins the anterior stomach in Amb III. The anterior stomach is characterized by the presence of two separate festoons and a large, adapically oriented dilation located near IAmb 2. McRae [23: 238] described the internal anatomy of Evechinus chloroticus and noted that the anterior stomach is characterized by the presence of a "sac-like dilatation" that is connected to esophagus and axial complex "through sheets of mesentery".

\section{Strongylocentrotidae}

The anterior stomach of Pseudocentrotus depressus, Strongylocentrotus droebachiensis (Figure 5I), and Strongylocentrotus purpuratus (Figures 5J; 9K) is located in Amb III. The moderately winding esophagus is initially directed towards the apex before descending towards IAmb 3; it later connects to the anterior stomach in Amb III. The two individual festoons in Amb III are horizontally fused for parts of their length. A small dilation occurs immediately distal to the connection of esophagus and stomach. This dilation is directed adapically and extends into IAmb 2.

\section{Toxopneustidae}

The species that so far have been analyzed in this taxon all possess a prominent anterior stomach located in Amb III. In Gymnechinus robillardi, Lytechinus variegatus, Nudechinus scotiopremnus, Sphaerechinus granularis (Figure 5K, L), Toxopneustes pileolus, Tripneustes esculentus, Tripneustes gratilla, and Tripneustes ventricosus, the two individual festoons of the anterior stomach are moderately fused and located in Amb III. The winding, short esophagus is initially directed towards the apex before descending towards IAmb 3; it later connects to the anterior stomach in Amb III. A variably sized dilation of the anterior stomach, largely located in IAmb 2 , is oriented adapically. The esophagus in Tripneustes spp. doubles back on itself [49].

\section{Irregularia}

Irregular sea urchins can be distinguished from "regular" species by the absence of festoons in their entire digestive tract (Figure 9L-T).

\section{Echinoneidae}

The anterior stomach of Echinoneus cyclostomus (Figures 6A, 9L, interactive Figure 10C) is located in Amb III and also extends into IAmb 3. The short esophagus ascends from the mouth towards Amb III where it joins the anterior stomach. A large pouch is located in Amb III distal to the junction of esophagus and anterior stomach. The pouch is connected to the anterior stomach through a small opening in the middle of the lower rim of the pouch. In lateral view, the pouch is triangular, with one apex towards the echinoid's anterior edge. The aboral edge of the pouch closely follows the arched form of the test in Amb III. This pouch extends from the anterior part of the stomach in Amb III, close to the ampullae of the tube feet, until it reaches the axial complex located in the central oral-aboral axis. The surface of the pouch is covered with numerous small folds. The pouch is broader at its adoral edge than towards the apex.

\section{Apatopygidae}

The anterior stomach of Apatoypgus recens (Figure 6B) is located in Amb III. The short esophagus ascends from the mouth towards Amb III where it joins the anterior stomach. The apical side of the anterior stomach is characterized by the presence of a large pouch that extends obliquely within Amb III from the anterior part close to the test, until it reaches the axial complex 
Table 3 List of irregular sea urchin taxa included in this study

\begin{tabular}{|c|c|c|c|c|c|}
\hline Order & Family & Species & Method used & Specimen ID & Reference \\
\hline $\begin{array}{l}\text { Echinoneoida Clark, } \\
1925\end{array}$ & $\begin{array}{l}\text { Echinoneidae Agassiz } \\
\text { \& Desor, } 1847\end{array}$ & $\begin{array}{l}\text { Echinoneus cyclostomus } \\
\text { Leske, } 1778\end{array}$ & $\begin{array}{l}\text { MRI }(66 \mu \mathrm{m})^{3}, \\
\text { dissection }\end{array}$ & NHM 1969.5.1.105, ZMB 4963 & [50], this study \\
\hline \multirow[t]{5}{*}{$\begin{array}{l}\text { Cassiduloida Agassiz \& } \\
\text { Desor, } 1847\end{array}$} & $\begin{array}{l}\text { Apatopygidae Kier, } \\
1962\end{array}$ & $\begin{array}{l}\text { Apatopygus recens } \\
\text { (Mortensen, 1948) }\end{array}$ & Dissection & ZMK Mortensen collection & this study \\
\hline & $\begin{array}{l}\text { Cassidulidae Agassiz \& } \\
\text { Desor, } 1847\end{array}$ & $\begin{array}{l}\text { Cassidulus caribaearum de } \\
\text { Lamarck, } 1801\end{array}$ & MRI $(81 \mu \mathrm{m})^{3}$ & CASIZ 112632 & [70], this study \\
\hline & & $\begin{array}{l}\text { Rhyncholampas pacificus } \\
\text { (Agassiz, 1863) }\end{array}$ & Dissection & - & [13] \\
\hline & $\begin{array}{l}\text { Echinolampadidae } \\
\text { Gray, } 1851\end{array}$ & $\begin{array}{l}\text { Echinolampas depressa } \\
\text { Gray, } 1851\end{array}$ & MRI $(81 \mu \mathrm{m})^{3}$ & USNM E32955 & this study \\
\hline & $\begin{array}{l}\text { Neolampadidae } \\
\text { Lambert, } 1918\end{array}$ & $\begin{array}{l}\text { Neolampas rostellata } \\
\text { Agassiz, } 1869\end{array}$ & $\begin{array}{l}\text { MRI }(81 \mu \mathrm{m})^{3}, \\
\text { dissection }\end{array}$ & MNHN EcEh 330 & this study \\
\hline \multirow[t]{22}{*}{$\begin{array}{l}\text { Clypeasteroida Agassiz, } \\
1835\end{array}$} & $\begin{array}{l}\text { Clypeasteridae } \\
\text { Agassiz, } 1835\end{array}$ & $\begin{array}{l}\text { Clypeaster annandalei } \\
\text { Koehler, } 1922\end{array}$ & Dissection & - & [51] \\
\hline & & $\begin{array}{l}\text { Clypeaster destinatus } \\
\text { Koehler, } 1922\end{array}$ & Dissection & - & [51] \\
\hline & & $\begin{array}{l}\text { Clypeaster europacificus } \\
\text { Clark, } 1914\end{array}$ & Dissection & CASIZ 101408 & this study \\
\hline & & $\begin{array}{l}\text { Clypeaster humilis (Leske, } \\
\text { 1778) }\end{array}$ & Dissection & - & [51] \\
\hline & & $\begin{array}{l}\text { Clypeaster rarispinus de } \\
\text { Meijere, } 1903\end{array}$ & Dissection & - & [51] \\
\hline & & $\begin{array}{l}\text { Clypeaster reticulatus } \\
\text { (Linnaeus, 1758) }\end{array}$ & MRI $(81 \mu \mathrm{m})^{3}$ & USNM 34282 & [51], this study \\
\hline & & $\begin{array}{l}\text { Clypeaster rosaceus } \\
\text { (Linnaeus, 1758) }\end{array}$ & $\begin{array}{l}\text { MRI }(96 \mu \mathrm{m})^{3}, \\
\text { dissection }\end{array}$ & ZMB 2520 & [13], this study \\
\hline & $\begin{array}{l}\text { Arachnoididae } \\
\text { Duncan, } 1889\end{array}$ & $\begin{array}{l}\text { Arachnoides placenta } \\
\text { (Linnaeus, 1758) }\end{array}$ & $\begin{array}{l}\text { MRI }(81 \mu \mathrm{m})^{3} \text {, } \\
\text { dissection }\end{array}$ & $\begin{array}{l}\text { ZMB 1439, CASIZ 93620, CASIZ } \\
\text { 103170, CASIZ } 94172\end{array}$ & [51], this study \\
\hline & $\begin{array}{l}\text { Laganidae Agassiz, } \\
1873\end{array}$ & $\begin{array}{l}\text { Laganum bonani Klein, } \\
1734\end{array}$ & Dissection & - & [51] \\
\hline & & $\begin{array}{l}\text { Laganum decagonale de } \\
\text { Blainville, } 1827\end{array}$ & Dissection & - & [51] \\
\hline & & $\begin{array}{l}\text { Laganum depressum } \\
\text { Agassiz, } 1841\end{array}$ & $\begin{array}{l}\text { MRI }(86 \mu \mathrm{m})^{3}, \\
\text { dissection }\end{array}$ & NHM 1932.4.28.227-34 & [51], this study \\
\hline & & $\begin{array}{l}\text { Laganum joubini (Koehler, } \\
\text { 1922) }\end{array}$ & MRI $(44 \mu \mathrm{m})^{3}$ & NHM 1979.1.25.52-60 & this study \\
\hline & & $\begin{array}{l}\text { Laganum laganum (Leske, } \\
1778 \text { ) }\end{array}$ & MRI $(81 \mu \mathrm{m})^{3}$ & CASIZ 94344 & [51], this study \\
\hline & & $\begin{array}{l}\text { Peronella lesueuri (Agassiz, } \\
\text { 1841) }\end{array}$ & $\begin{array}{l}\text { MRI }(81 \mu \mathrm{m})^{3}, \\
\text { dissection }\end{array}$ & MNHN EcEh 79 & [51], this study \\
\hline & & $\begin{array}{l}\text { Peronella orbicularis Leske, } \\
1778\end{array}$ & $\begin{array}{l}\text { MRI }(81 \mu \mathrm{m})^{3}, \\
\text { dissection }\end{array}$ & MNHN EcEh 77 & {$[52]$, this study } \\
\hline & Fibulariidae Gray, 1855 & $\begin{array}{l}\text { Echinocyamus pusillus } \\
\text { (Müller, 1776) }\end{array}$ & $\begin{array}{l}\text { MRI } 20 \times 18 \times \\
18 \mu^{3}\end{array}$ & - & {$[52]$, this study } \\
\hline & Rotulidae Gray, 1855 & $\begin{array}{l}\text { Fibulariella acuta } \\
\text { (Yoshiwara, 1898) }\end{array}$ & Dissection & CASIZ uncataloged material & this study \\
\hline & & $\begin{array}{l}\text { Rotula deciesdigitata (Leske, } \\
\text { 1778) }\end{array}$ & $\begin{array}{l}\text { MRI }(81 \mu \mathrm{m})^{3}, \\
\text { dissection }\end{array}$ & ZMB 2169 & [53], this study \\
\hline & $\begin{array}{l}\text { Echinarachniidae } \\
\text { Lambert, } 1914\end{array}$ & $\begin{array}{l}\text { Echinarachnius parma (de } \\
\text { Lamarck, 1816) }\end{array}$ & Dissection & ZSM 20011676, CASIZ 157683 & $\begin{array}{l}{[40,45,51,54], \text { this }} \\
\text { study }\end{array}$ \\
\hline & $\begin{array}{l}\text { Dendrasteridae } \\
\text { Lambert, } 1900\end{array}$ & $\begin{array}{l}\text { Dendraster excentricus } \\
\text { (Eschscholtz, 1831) }\end{array}$ & Dissection & - & [54-58], this stud) \\
\hline & & $\begin{array}{l}\text { Scaphechinus griseus } \\
\text { (Mortensen, 1927) }\end{array}$ & Dissection & - & {$[59]$} \\
\hline & & $\begin{array}{l}\text { Scaphechinus mirabilis } \\
\text { Agassiz, } 1863\end{array}$ & Dissection & - & {$[59]$} \\
\hline
\end{tabular}


Table 3 List of irregular sea urchin taxa included in this study (Continued)

\begin{tabular}{|c|c|c|c|c|c|}
\hline & & $\begin{array}{l}\text { Scaphechinus tenuis } \\
\text { (Yoshiwara, 1898) }\end{array}$ & Dissection & CASIZ 110668 & this study \\
\hline & & $\begin{array}{l}\text { Sinaechinocyamus mai } \\
\text { (Wang, 1984) }\end{array}$ & Dissection & CASIZ uncatalogued material & this study \\
\hline & $\begin{array}{l}\text { Astriclypeidae } \\
\text { Stefanini, } 1912\end{array}$ & $\begin{array}{l}\text { Astriclypeus mannii Verrill, } \\
1867\end{array}$ & Dissection & - & this study \\
\hline & & $\begin{array}{l}\text { Echinodiscus auritus Leske, } \\
1778\end{array}$ & Dissection & - & {$[51]$} \\
\hline & $\begin{array}{l}\text { Mellitidae Stefanini, } \\
1912\end{array}$ & $\begin{array}{l}\text { Encope stokesii Agassiz, } \\
1841\end{array}$ & Dissection & CASIZ 3387 & this study \\
\hline & & $\begin{array}{l}\text { Leodia sexiesperforata } \\
\text { (Leske, 1778) }\end{array}$ & Dissection & - & {$[59]$} \\
\hline & & $\begin{array}{l}\text { Mellita quinquiesperforata } \\
\text { (Leske, 1778) }\end{array}$ & Dissection & - & $\begin{array}{l}{[40,54,60,61] \text {, this }} \\
\text { study }\end{array}$ \\
\hline \multirow[t]{7}{*}{$\begin{array}{l}\text { Holasteroida Durham \& } \\
\text { Melville, } 1957\end{array}$} & $\begin{array}{l}\text { Corystidae Foster \& } \\
\text { Philip, } 1978\end{array}$ & $\begin{array}{l}\text { Corystus relictus (de } \\
\text { Meijere, 1902) }\end{array}$ & Dissection & ZMK Mortensen collection & this study \\
\hline & $\begin{array}{l}\text { Urechinidae Duncan, } \\
1889\end{array}$ & $\begin{array}{l}\text { Antrechinus nordenskjoldi } \\
\text { (Mortensen, 1905) }\end{array}$ & Dissection & ZMH E7350 & this study \\
\hline & & $\begin{array}{l}\text { Urechinus naresianus } \\
\text { Agassiz, } 1879\end{array}$ & Dissection & NHM 1903.8.1.100-104 & {$[28,62]$, this study } \\
\hline & $\begin{array}{l}\text { Plexechinidae Mooi \& } \\
\text { David, } 1996\end{array}$ & $\begin{array}{l}\text { Plexechinus aoteanus } \\
\text { McKnight, } 1974\end{array}$ & Dissection & ZMH E7345 & this study \\
\hline & $\begin{array}{l}\text { Pourtalesiidae Agassiz, } \\
1881\end{array}$ & $\begin{array}{l}\text { Pourtalesia hispida Agassiz, } \\
1879\end{array}$ & Dissection & ZMH E7349 & this study \\
\hline & & $\begin{array}{l}\text { Pourtalesia jeffreysi Wyville } \\
\text { Thomson, } 1872\end{array}$ & $\begin{array}{l}\text { MRI }(81 \mu \mathrm{m})^{3} \text {, } \\
\text { dissection }\end{array}$ & ZSM 20011456 & [62], this study \\
\hline & & $\begin{array}{l}\text { Pourtalesia wandeli } \\
\text { Mortensen, } 1905\end{array}$ & MRI $(86 \mu \mathrm{m})^{3}$ & NHM 1976.7.30.76-95 & this study \\
\hline \multirow[t]{14}{*}{$\begin{array}{l}\text { Spatangoida Agassiz, } \\
1840\end{array}$} & $\begin{array}{l}\text { Aeropsidae Lambert, } \\
1896\end{array}$ & $\begin{array}{l}\text { Aeropsis fulva (Agassiz, } \\
\text { 1881) }\end{array}$ & Dissection & CASIZ 113902 & this study \\
\hline & $\begin{array}{l}\text { Hemiasteridae Clark, } \\
1917\end{array}$ & $\begin{array}{l}\text { Hemiaster expergitus } \\
\text { (Lovén, 1874) }\end{array}$ & Dissection & NHM 1914.1.30.66-9 & this study \\
\hline & & $\begin{array}{l}\text { Hemiaster hickmanni } \\
\text { Koehler, } 1914\end{array}$ & Dissection & - & {$[63]$} \\
\hline & $\begin{array}{l}\text { Paleopneustidae } \\
\text { Agassiz, } 1904\end{array}$ & $\begin{array}{l}\text { Paleopneustes cristatus } \\
\text { Agassiz, } 1873\end{array}$ & Dissection & - & {$[64]$} \\
\hline & & $\begin{array}{l}\text { Paleopneustes tholoformis } \\
\text { Chesher, } 1968\end{array}$ & Dissection & - & {$[64]$} \\
\hline & $\begin{array}{l}\text { Prenasteridae Lambert, } \\
1905\end{array}$ & $\begin{array}{l}\text { Prenaster enodatus } \\
\text { (Chesher, 1968) }\end{array}$ & Dissection & - & {$[64]$} \\
\hline & $\begin{array}{l}\text { Schizasteridae } \\
\text { Lambert, } 1905\end{array}$ & $\begin{array}{l}\text { Abatus cavernosus (Philippi, } \\
1845 \text { ) }\end{array}$ & MRI $(81 \mu \mathrm{m})^{3}$ & ZMB 5854 & this study \\
\hline & & $\begin{array}{l}\text { Abatus cordatus (Verrill, } \\
\text { 1876) }\end{array}$ & Dissection & ZMB 5437 & this study \\
\hline & & $\begin{array}{l}\text { Aceste ovata Agassiz \& } \\
\text { Clark, } 1907\end{array}$ & Dissection & - & {$[63]$} \\
\hline & & $\begin{array}{l}\text { Brisaster antarcticus } \\
\text { (Döderlein, 1906) }\end{array}$ & Dissection & AAD uncataloged material & this study \\
\hline & & $\begin{array}{l}\text { Brisaster fragilis (Duben \& } \\
\text { Koren, 1846) }\end{array}$ & Dissection & ZMB 2766 & this study \\
\hline & & $\begin{array}{l}\text { Brisaster latifrons (Agassiz, } \\
\text { 1898) }\end{array}$ & Dissection & - & $\begin{array}{l}\text { Sampson } \\
\text { (unpublished } \\
\text { data) }\end{array}$ \\
\hline & & $\begin{array}{l}\text { Hypselaster kempi (Koehler, } \\
\text { 1914) }\end{array}$ & Dissection & - & {$[63]$} \\
\hline & & $\begin{array}{l}\text { Schizaster canaliferus (de } \\
\text { Lamarck, 1816) }\end{array}$ & Dissection & - & {$[9,65]$} \\
\hline
\end{tabular}


Table 3 List of irregular sea urchin taxa included in this study (Continued)

\begin{tabular}{|c|c|c|c|c|}
\hline Brissidae Gray, 1855 & $\begin{array}{l}\text { Brissus agassizii Döderlein, } \\
1885\end{array}$ & Dissection & - & this study \\
\hline & $\begin{array}{l}\text { Brissus unicolor (Leske, } \\
\text { 1778) }\end{array}$ & Dissection & - & {$[9,66]$} \\
\hline & $\begin{array}{l}\text { Meoma ventricosa } \\
\text { (Lamarck, 1816) }\end{array}$ & Dissection & - & [67], this study \\
\hline & $\begin{array}{l}\text { Metalia sternalis Lamarck, } \\
1816\end{array}$ & Dissection & - & [13] \\
\hline $\begin{array}{l}\text { Brissopsidae Lambert, } \\
1905\end{array}$ & $\begin{array}{l}\text { Brissopsis alta Mortensen, } \\
1907\end{array}$ & Dissection & - & {$[64]$} \\
\hline & $\begin{array}{l}\text { Brissopsis atlantica } \\
\text { Mortensen, } 1907\end{array}$ & Dissection & - & [64] \\
\hline & $\begin{array}{l}\text { Brissopsis elongata } \\
\text { Mortensen, } 1907\end{array}$ & Dissection & - & [64] \\
\hline & $\begin{array}{l}\text { Brissopsis lyrifera (Forbes, } \\
\text { 1841) }\end{array}$ & Dissection & ZMB 7259 & {$[9,64]$, this study } \\
\hline & $\begin{array}{l}\text { Brissopsis mediterranea } \\
\text { Mortensen, } 1913\end{array}$ & Dissection & - & [64] \\
\hline $\begin{array}{l}\text { Loveniidae Lambert, } \\
1905\end{array}$ & $\begin{array}{l}\text { Echinocardium cordatum } \\
\text { (Pennant, 1777) }\end{array}$ & $\begin{array}{l}\text { MRI }(81 \mu \mathrm{m})^{3}, \\
\text { dissection }\end{array}$ & - & $\begin{array}{l}{[12,15,68], \text { this }} \\
\text { study }\end{array}$ \\
\hline & $\begin{array}{l}\text { Echinocardium flavescens } \\
\text { (Müller, 1776) }\end{array}$ & Dissection & - & [9] \\
\hline & $\begin{array}{l}\text { Lovenia subcarinata (Gray, } \\
\text { 1845) }\end{array}$ & Dissection & - & [4] \\
\hline $\begin{array}{l}\text { Spatangidae Gray, } \\
1825\end{array}$ & $\begin{array}{l}\text { Plethotaenia angularis } \\
\text { Chesher, } 1968\end{array}$ & Dissection & - & {$[64]$} \\
\hline & $\begin{array}{l}\text { Plethotaenia spatangoides } \\
\text { Agassiz, } 1883\end{array}$ & Dissection & - & [64] \\
\hline & $\begin{array}{l}\text { Pseudomaretia alta } \\
\text { (Agassiz, 1863) }\end{array}$ & MRI $(81 \mu \mathrm{m})^{3}$ & ZSM 20011608 & this study \\
\hline & $\begin{array}{l}\text { Spatangus purpureus } \\
\text { Müller, } 1776\end{array}$ & $\begin{array}{l}\text { MRI }(81 \mu \mathrm{m})^{3}, \\
\text { dissection }\end{array}$ & ZMB 3236 & $\begin{array}{l}{[8,9,17,66], \text { this }} \\
\text { study }\end{array}$ \\
\hline $\begin{array}{l}\text { Maretiidae Lambert, } \\
1905\end{array}$ & $\begin{array}{l}\text { Maretia planulata (de } \\
\text { Lamarck, 1816) }\end{array}$ & Dissection & - & [4] \\
\hline $\begin{array}{l}\text { Asterostomatidae } \\
\text { Pictet, } 1857\end{array}$ & $\begin{array}{l}\text { Elipneustes denudatus } \\
\text { (Koehler, 1914) }\end{array}$ & Dissection & - & [63] \\
\hline & $\begin{array}{l}\text { Heterobrissus hemingi } \\
\text { (Anderson, 1902) }\end{array}$ & Dissection & - & [63] \\
\hline & $\begin{array}{l}\text { Heterobrissus niasicus } \\
\text { (Döderlein, 1901) }\end{array}$ & Dissection & - & [69] \\
\hline
\end{tabular}

The table provides information on every species studied so far with regard to digestive tract anatomy, listing the method(s) used, the specimen ID of museum specimens where applicable, and the respective references. Numbers in brackets behind "MRI" represent the resolution of the dataset. An overview of scanning parameters is provided by $[25,86]$. this study = specimens were dissected and/or scanned in the course of this study; see the 'List of abbreviations used' section for an explanation of abbreviations.

that bulges anteriorly. The pouch is connected to the anterior stomach through a broad canal located at the anterior end of its adoral edge. The general form of the pouch is triangular, the anterior point being located in Amb III. The surface of the pouch is characterized by numerous folds running along the oral-aboral axis. The aboral edge of the pouch closely follows the arched form of the test in Amb III.

\section{Cassidulidae}

In Cassidulus caribaearum (Figure 9M) and Rhyncholampas pacificus (Figure 6C), the anterior stomach is located in Amb III and IAmb 3. The short esophagus ascends from the mouth towards Amb III until it joins the anterior stomach. At the junction of esophagus and anterior stomach, a cluster of small, smooth, finger-like pouches can be found. These pouches are directed towards the apex of Amb III and split into two smaller clusters, one directed laterally towards IAmb 2, the other oriented towards IAmb 3. The number of pouches in an adult specimen of Cassidulus caribaearum was found to be approximately four to six on each side. The apical side of the entire structure is in close proximity 
to the apical part of the test of Amb III. Agassiz [13] and Gladfelter [70] provided similar descriptions of this cluster of pouches located at the apex of the anterior stomach.

\section{Echinolampadidae}

The anterior stomach of Echinolampas depressa (Figure $9 \mathrm{~N}$ ) is primarily located in IAmb 3 , but reaches into Amb III as well. A short esophagus ascends from the mouth towards Amb III where it joins the anterior stomach. At the apical part of the anterior stomach, a cluster of small, smooth, pointed pouches are located. As in Cassidulus, these pouches are directed towards the apex of Amb III and later divide to form two smaller clusters that are directed laterally, one towards IAmb 2, the other towards IAmb 3. The pouches are wedged between the upper coil of the digestive tract and are not in close proximity to the apical part of the test in Amb III. The number of pouches in an adult specimen of Echinolampas depressa was found to be approximately eight to twelve on each side.

\section{Neolampadidae}

The digestive tract of the single specimen of Neolampas rostellata (Figure $6 \mathrm{D}$ ) that could be analyzed in the course of this study is characterized by the presence of the anterior stomach in Amb III. The short esophagus ascends from the mouth towards the anterior stomach, joining it in Amb III. A considerable dilation or pouch can be found branching off the anterior stomach towards IAmb 2. On the apical side of this structure, another knob-like dilation or pouch can be found. Its surface consists of several smooth, finger-like folds that adhere to each other.

\section{Clypeasteridae}

The anterior stomach of all species within the genus Clypeaster that have been analyzed so far is located in Amb III and IAmb 3. The short esophagus, originating from the top of Aristotle's lantern, is directed towards Amb III where it joins the anterior stomach. Distal to this junction, a grape-like cluster of pouches is present both in juvenile specimens of Clypeaster europacificus and adult specimens of Clypeaster annandalei, Clypeaster destinatus (Figure 6E) (Mortensen 71 regards the former two species as synonymous), Clypeaster humilis, Clypeaster rarispinus (Figure 6F), Clypeaster reticulatus (Figure 9O), and Clypeaster rosaceus. This cluster extends along the anterior stomach towards the anterior part of the animal in Amb III, and consists of dozens of smooth, grape-like nodules. Koehler [51: 27] described this structure as a "glande intestinale".

\section{Arachnoididae}

The anterior stomach in Arachnoides placenta (Figure 6G, H) is located in Amb III and reaches into Amb 2. A short esophagus connects the pharynx with the anterior stomach in Amb III. Between the central oral-aboral axis and the anterior tip of the test in Amb III, a cluster of pouches shaped like bunches of grapes extends laterally from the anterior stomach into IAmb 2 . This cluster is present both in juvenile (Figure 6G) and adult specimens (Figure $6 \mathrm{H}$ ), although its relative size seems to increase with age. The surface of each of the numerous individual pouches is smooth; some are more elongated than others. In adult specimens (ca. $10 \mathrm{~cm}$ test diameter), the entire cluster may attain a length of approximately $2 \mathrm{~cm}$. Koehler [51:27] described this structure as a "glande intestinale".

\section{Laganidae}

The anterior stomach in Laganum bonani, Laganum decagonale, Laganum depressum, Laganum joubini, Laganum laganum (Figures 7A, 9P), as well as in Peronella lesueuri and Peronella orbicularis (Figure 7B) is located in Amb III and IAmb 2, in some species extending well into Amb II. A short and broad esophagus reaches from the surface of Aristotle's lantern towards the anterior stomach in IAmb 2. The antero-lateral edge of the anterior stomach is slightly lobate in some species, particularly so in Laganum laganum. No particular dilation or pouch of the anterior stomach was observed in members of this taxon. Mortensen [71:246] stated that in the Laganidae "...the intestinal gland appears to be lacking completely, in contradistinction to the Clypeastrids proper and Arachnoides."

\section{Fibulariidae}

The digestive tract of Echinocyamus pusillus (Figure 7C) is characterized by the presence of the anterior stomach at the border of Amb II and IAmb 2. The antero-lateral edge of the anterior stomach is smooth. The short esophagus connects the pharynx from the apical surface of Aristotle's lantern to the anterior stomach in IAmb 2. Dilations or pouches are absent from the anterior stomach.

\section{Rotulidae}

The anterior stomach of Rotula deciesdigitata (Figure 9Q) is located between Amb II and Amb III. A short esophagus spans from the surface of Aristotle's lantern towards the anterior stomach located in IAmb 2. The antero-lateral edge of the anterior stomach is smooth. Koehler [53] described the anterior stomach of Rotula deciesdigitata as considerably enlarged in comparison to the rest of the stomach. No dilations or pouches could be observed in the anterior stomach. Recent analysis of the subgenus Fibulariella (sensu Mortensen [71]) indicates that it is most closely related to the rotulids, not the fibulariids [72]. Accordingly, we report here that in Fibulariella acuta (Figure 7D), the configuration of the anterior stomach is similar to that in Rotula.

\section{Echinarachniidae}

The anterior stomach in Echinarachnius parma (Figures 7E, F; 9R) is located between Amb II and III. A short, 
broad esophagus connects the pharynx with the anterior stomach in IAmb 2. The abaxial edge of most parts of the stomach is characterized by the presence of a conspicuously frilled zone (Figure 7F) that can reach from IAmb 2 as far as IAmb 4. No particular dilation or pouch of the anterior stomach was observed.

\section{Dendrasteridae}

Data on the internal anatomy of dendrasterid sand dollars are available for Scaphechinus griseus, Scaphechinus mirabilis, Scaphechinus tenuis (Figure 7G), Dendraster excentricus (Figure 7H), and Sinaechinocyamus mai (Figure 7I). The anterior stomach is located between IAmb 2 and Amb III. A short, broad esophagus connects the pharynx with the anterior stomach at the border of Amb III and IAmb 2. Dilations or pouches of the anterior stomach could not be found. According to Reisman [55: 8], the stomach of Dendraster excentricus "is differentiated into a narrow, brown, fluted outer region and a wide mustard-yellow less fluted inner region."

\section{Astriclypeidae}

The digestive tract in Astriclypeus manni and Echinodiscus auritus (Figure 7J) is characterized by the presence of an anterior stomach located in IAmb 2 and Amb III. The short esophagus connects the pharynx with the anterior stomach in Amb III. The abaxial edge of most parts of the stomach consists of a smooth to frilled area that reaches from Amb III as far as Amb V. No particular dilation or pouch was observed in members of this taxon.

\section{Mellitidae}

The anterior stomach in Encope stokesii (Figure 7K), Leodia sexiesperforata, and Mellita quinquiesperforata (Figure 7L) is located in IAmb 2 and Amb III. A short esophagus connects pharynx with anterior stomach at the border of IAmb 2 and Amb III. The abaxial edge of parts of the stomach is characterized by the presence of a conspicuously frilled zone (Figure $7 \mathrm{~L}$ ) that may reach from IAmb 2 as far as Amb IV. The anterior stomach is devoid of any dilations or pouches.

\section{Atelostomata}

Members of the following families all belong to a monophyletic taxon, the Atelostomata. All are characterized by the absence of Aristotle's lantern during all ontogenetic stages. Furthermore, the esophagus is directed towards the posterior part of the animal, i.e. IAmb 5, where it curves counter-clockwise until about IAmb 1, the branching-off point of the large primary siphon. Whether the subsequent part of the digestive tract from IAmb 1 to Amb III is derived from the esophagus or the stomach is currently a matter of debate. In the present study, we assume that the point at which the primary siphon branches off marks the end of the esophagus, implying that the anterior stomach has stretched from Amb III towards IAmb 1. This in turn creates a digestive tract area not present in non-atelostomate taxa. Histological and ultrastructural data support this view, but such data are patchy and currently available only for a limited number of taxa $[4,8,9,12,69]$.

\section{Corystidae}

The anterior stomach in Corystus relictus (Figure 8A) extends from IAmb 1 until about IAmb 3. A large pouch is located on top part of the anterior stomach and extends clockwise from IAmb 3 until IAmb 5. Its connection with the anterior stomach is located in IAmb 3. The surface of the pouch is smooth.

\section{Urechinidae}

The anterior stomach in Antrechinus nordenskjoldi (Figure 8B) and Urechinus naresianus (Figure 8C, D) extends from IAmb 1 until about IAmb 3. A large pouch is located on top of the first part of the anterior stomach, extending from its connection to the former in Amb III clockwise towards IAmb 1. The surface of the pouch may be smooth (Figure $8 \mathrm{~B}$ ) or slightly lobate (Figure 8C). Mortensen [62: 42] states, that a "well developed diverticulum" is present in Urechinus naresianus.

\section{Plexechinidae}

The anterior stomach of Plexechinus aoteanus is characterized by the presence of a large pouch that connects to the underlying anterior stomach in Amb III.

\section{Pourtalesiidae}

The digestive tract of Pourtalesia jeffreysi (Figure 8E), Pourtalesia hispida (Figure 8F), and Pourtalesia wandeli (Figure 9S) is characterized by an anterior stomach that stretches between IAmb 1 and IAmb3. A large pouch is located on top of the first part of the anterior stomach, pointing from its connection to the former in Amb III straight towards IAmb 5. The apical surface of the pouch is smooth, whereas its sides as well as the adoral surface are lobate. According to Mortensen [62: 62], "the blind diverticulum is well developed, lobate" in the Pourtalesiidae.

\section{Aeropsidae}

The anterior stomach of Aeropsis fulva (Figure 8G) stretches approximately from IAmb 1 to IAmb 3. The esophagus is thin and joins the stomach presumably in IAmb 1. No particular dilation or pouch was observed in the vicinity of the anterior stomach. Although Agassiz [36] depicted a lateral view of the internal anatomy of Aeropsis rostrata, he unfortunately did not specifically mention the absence or presence of a pouch in the respective area of the digestive tract.

\section{Hemiasteridae}

The anterior stomach in Hemiaster expergitus (Figure $8 \mathrm{H}$ ) is located between IAmb 1 and IAmb 3. A large pouch is located on top of the first part of the anterior stomach, pointing from its connection to the former in 
Amb III straight towards IAmb 5. The apical surface of the pouch is smooth, whereas its sides as well as the adoral surface are lobate. Koehler [63] mentions a long and only very slightly lobate pouch to be present at the same location in Hemiaster hickmanni.

\section{Palaeopneustidae}

In his account on various spatangoid species, Chesher [64] described the internal anatomy of Spatangus purpureus (see Spatangidae below), also mentioning the presence of a large pouch on top of the anterior stomach. For Paleopneustes cristatus and Paleopneustes tholoformis he noted that their internal anatomy largely resembled that of Spatangus purpureus, implying that there is a pouch present on top of the anterior stomach in these two palaeopneustid species as well.

\section{Prenasteridae}

Similar to his observations on palaeoneustid species, Chesher [64] noted that the internal anatomy of Prenaster enodatus largely resembled that of Spatangus purpureus, implying that there is a pouch present on top of the anterior stomach in this species as well.

\section{Schizasteridae}

The anterior stomach in Schizaster canaliferus, Abatus cavernosus (Figure 9T), Abatus cordatus, Brisaster antarcticus, Brisaster fragilis, and Brisaster latifrons (Figure $8 \mathrm{I}$ ) is located between IAmb 1 and IAmb 3. A large pouch is located on top of the first part of the anterior stomach, pointing from its connection to the former in Amb III straight towards IAmb 5. The apical surface of the pouch is smooth, whereas its sides as well as the adoral surface are lobate. However, Koehler [9] described the pouch in Schizaster canaliferus as having simple, flat, and smooth walls. Later [63], he described the internal anatomy of other schizasterids and noted that the digestive tract in Hypselaster kempi is similar to the digestive tract in Hemiaster hickmanni, including the presence of a large pouch on top of the anterior stomach. On the other hand, Koehler [63] mentioned that there is no gastric caecum (= pouch in this description) in Aceste ovata.

\section{Brissidae}

The anterior stomach in Brissus agassizii, Brissus unicolor and Meoma ventricosa (Figure 8J, K) is located between IAmb 1 and IAmb 3. A large pouch is located on top of the first part of the anterior stomach, pointing from its connection to the former in IAmb 3 towards IAmb 5 in an oblique manner. The apical surface of the pouch is smooth, whereas its sides as well as the aboral surface are lobate. According to Chesher [67,: 99], the pouch is a "highly vascularized, thin, convoluted sac which occupies a major portion of the coelom between intestine and the gonads. Sand does not enter the caecum." Agassiz [13: 677] gave a differing description of the anterior stomach in another brissid, Metalia sternalis: "...at the junction of the esophagus with the alimentary canal proper is found a cluster of small diverticula resembling those of Rhynchopygus, and not a single large diverticulum as in Spatangus proper." (Rhynchopygus $=$ Rhyncholampas in the present article, see Cassidulidae).

\section{Brissopsidae}

The anterior stomach in Brissopsis lyrifera (Figure 8L) is located between IAmb 1 and IAmb 3. A large pouch is located on top of the first part of the anterior stomach, pointing from its connection to the former in IAmb 3 towards IAmb 5 in an oblique form. The apical surface of the pouch is smooth, whereas its sides as well as the adoral surface are lobate. According to Chesher [64], the following taxa closely resemble Brissopsis lyrifera with regard to internal anatomy, implying the presence of a pouch on top of the anterior stomach: Brissopsis alta, Brissopsis atlantica, Brissopsis elongata, and Brissopsis mediterranea.

\section{Loveniidae}

The anterior stomach in Echinocardium cordatum (Figure $8 \mathrm{M}$ ) is located between IAmb 1 and IAmb 3. A large pouch is located on top of the first part of the anterior stomach, pointing from its connection to the former in IAmb 3 towards IAmb 5 in an oblique form. The apical surface of the pouch is smooth, whereas its sides as well as the adoral surface are lobate. According to De Ridder \& Jangoux [12: 338], the anterior stomach of Echinocardium cordatum is characterized by the presence of "a large, translucent, turgid (fluid-filled), and non-contractile triangular pouch". Their analyses reveal that "no muscular sphincter is associated with the caecal opening, but a small prominence of dense connective tissue occurs at the level of the caecal slit. This prominence locally brings both faces of the caecal slit closer to each other, the opening never being tightly closed". Koehler [9] briefly described the internal anatomy of Echinocardium cordatum as well as Echinocardium flavescens and noted the presence of a large pouch with flat, simple, and smooth walls. Furthermore, Holland \& Ghiselin [4] mentioned the presence of a large pouch atop the anterior stomach in Lovenia subcarinata.

\section{Spatangidae}

The anterior stomach in Spatangus purpureus (Figures $1 \mathrm{~A}, \mathrm{C} ; 8 \mathrm{~N}, \mathrm{O}$ ) is located between IAmb 1 and IAmb 3. A large pouch is located on top of the first part of the anterior stomach, pointing from its connection to the former in IAmb 3 towards IAmb 5 in an oblique form. The apical surface of the pouch is smooth, whereas its sides as well as the adoral surface are lobate. Several authors described the pouch on top of the anterior stomach in Spatangus purpureus $[8,9,13,14,17,18]$. For example, Agassiz [13: 677] described the pouch as a "... huge diverticulum, trending upwards and towards the 
posterior extremity...", while Koehler [9] noted that the connection between the anterior stomach and the pouch appeared as a narrow elliptical orifice. He also mentioned the presence of numerous transverse folds in the walls of the pouch. Finally, Henry [17: 1318] stated that “...l'intestin du Spatangus est absolument bourré de sable et de coquilles fines, le caecum, au contraire, ne contient pas un grain de sable...". Data on the internal morphology of spatangids are available for two additional species, Plethotaenia angularis and Plethotaenia spatangoides: according to Chesher [64], their digestive tract anatomy closely resembles that of Spatangus purpureus, indicating the presence of a large pouch. MRI data reveal the presence of a smaller pouch atop the anterior stomach in Pseudomaretia alta. In this species, the pouch is connected to the exterior part of the anterior stomach in IAmb 3 and extends in an oblique form towards Amb II.

\section{Maretiidae}

Holland \& Ghiselin [4] described the presence of a large pouch on top of the anterior stomach in Maretia planulata.

\section{Asterostomatidae}

The anterior stomach in Heterobrissus niasicus (Figure $8 \mathrm{P}$ ) is located between IAmb 1 and IAmb 3. A large pouch is located atop the first part of the anterior stomach, pointing from its connection to the former in IAmb 3 towards IAmb 5 in an oblique form. The apical surface of the pouch is smooth, whereas its sides as well as the adoral surface are lobate. Wagner [69: 35] described the pouch in Heterobrissus niasicus in detail and noted that "...das (erste) Divertikel ist ein breiter Blindsack, der an der Außenseite der unteren Darmwindung im Radius III entspringt..." and "...es ist von unten nach oben abgeplattet, und seine Oberfläche ist von zahlreichen Falten bedeckt...". Koehler [63] briefly mentioned the internal organization of Heterobrissus hemingi and stated - based on Wagner's detailed description that it is very similar to that in Heterobrissus niasicus. He furthermore noted the presence of a long, slim pouch on top of the anterior stomach in Elipneustes denudatus, whose general internal anatomy also closely resembled that of Heterobrissus niasicus.

\section{Discussion}

Our conclusions are possible through comprehensive analyses of 168 sea urchin species representing almost all extant sea urchin families. This approach entails the combined use of invasive and non-invasive techniques, which permits to reconsider data provided in publications dating back almost 200 years. However, in many cases our observations had to be based upon single specimens due to the scarcity of certain species, especially those from the deep sea. It should be noted that the exact delineation of digestive tract structures such as the esophagus, stomach, intestine, and rectum is still under debate. Holland \& Ghiselin [4] homologized substructures based on histological analyses. However, Jensen [6] mentioned - similar to Lewis' observations on Diadema antillarum [24] - that she had found valves within the digestive tract that would permit recognition of unequivocal homologies of sea urchin digestive tract compartments. Unfortunately, her data have never been published, so we base our designations primarily on Holland \& Ghiselin's scheme [4].

\section{Criteria employed to homologize substructures of the sea urchin anterior stomach}

In this section, we present a number of homology hypotheses that apply to all sea urchin taxa analyzed in this study: (i) presence or absence of festoons and of a gastric caecum in ambulacrum III; (ii) shape and size of the gastric caecum; (iii) mesenterial suspension of the anterior stomach and any substructures; and (iv) integration of the anterior stomach and any substructures into the haemal system of the digestive tract. The following sections include generalisations that are necessary in order to reveal the underlying homologies. However, we are aware that a certain degree of intraspecies variability present in sea urchins could result in slightly differing conclusions. A condensed compilation of the findings presented in this section is available in Table 4.

\section{(i) Absence or presence of festoons and of a gastric caecum} in the anterior stomach

The entire stomach is always more or less festooned in "regular" sea urchins (Figure 9A-K), whereas in irregular sea urchins these vertical inflections are absent (Figure 9L-T). In "regular" and basal irregular sea urchin species, the anterior stomach is always located in Amb III or in Amb III and its adjoining interambulacra. In contrast, in the Atelostomata (Figure 9S, T), the anterior stomach extends from about Amb I to at least IAmb 3, although the exact homologies are still a matter of debate $[4,6,12,15]$. The number of festoons in Amb III may vary from none in the Irregularia, a single one in the Cidaroida (at the border of Amb III and IAmb3), to two in the "regular" Euechinoidea (at the borders of Amb III and IAmb 2 as well as Amb III and IAmb 3). This finding implies that the Cidaroida generally possess nine stomach festoons in total, whereas the "regular" Euechinoidea possess ten stomach festoons. Apart from the additional festoon in Amb III, the "regular" Euechinoidea deviate from the cidaroid scheme by the presence of a more or less developed dilation lateral to the additional festoon located at the border of Amb III and IAmb 2. It is this dilation that in the Irregularia is present in the form of a pouch, and we therefore 
Table 4 Compilation of the primary morphological findings of this study related to the sea urchin anterior stomach in the form of a character matrix

\begin{tabular}{|c|c|c|c|c|c|c|c|c|c|c|c|c|c|}
\hline Taxon/Character & 1 & 2 & 3 & 4 & 5 & 6 & 7 & 8 & 9 & 10 & 11 & 12 & 13 \\
\hline Histocidaridae & 0 & 1 & 0 & - & 0 & 0 & - & - & - & - & - & 0 & - \\
\hline Ctenocidaridae & 0 & 1 & 0 & - & 0 & 0 & - & - & - & - & - & 0 & - \\
\hline Cidaridae & 0 & 1 & 0 & - & 0 & 0 & - & - & - & - & - & 0 & - \\
\hline Psychocidaridae & 0 & 1 & 0 & - & 0 & 0 & - & - & - & - & - & 0 & - \\
\hline Phormosomatidae & 0 & 1 & 1 & 0 & 0 & 1 & 0 & - & 0 & - & 0 & 0 & 0 \\
\hline Echinothuriidae & 0 & 1 & 1 & 0 & 0 & 1 & 0 & - & 0 & - & 0 & 0 & 0 \\
\hline Pedinidae & 0 & 1 & 1 & $0 / 1$ & 0 & 1 & 0 & - & 0 & - & 0 & 0 & 0 \\
\hline Micropygidae & 0 & 1 & 1 & 0 & 0 & 1 & 0 & - & 0 & - & 0 & 0 & 0 \\
\hline Aspidodiadematidae & 0 & 1 & 1 & 0 & 0 & 1 & 0 & - & 0 & - & 0 & 0 & 0 \\
\hline Diadematidae & 0 & 1 & 1 & 0 & 0 & 1 & 0 & - & 0 & - & 0 & 1 & 1 \\
\hline Glyptocidaridae & 0 & 1 & 1 & 0 & 0 & 1 & 0 & - & 0 & - & 0 & 0 & 0 \\
\hline Stomopneustidae & 0 & 1 & 1 & 0 & 0 & 1 & 0 & - & 0 & - & 0 & 0 & 0 \\
\hline Arbaciidae & 0 & 1 & 1 & 1 & 0 & 1 & 0 & - & 0 & - & 0 & 0 & 0 \\
\hline Saleniidae & 0 & 1 & 1 & 1 & 0 & 1 & 0 & - & 0 & - & 0 & 0 & 0 \\
\hline Temnopleuridae & 0 & 1 & 1 & 1 & 0 & 1 & 0 & - & 0 & - & 0 & 0 & 0 \\
\hline Trigonocidaridae & 0 & 1 & 1 & 1 & 0 & 1 & 0 & - & 0 & - & 0 & 0 & 0 \\
\hline Parasaleniidae & 0 & 1 & 1 & 0 & 0 & 1 & 0 & - & 0 & - & 0 & 0 & 0 \\
\hline Parechinidae & 0 & 1 & 1 & 1 & 0 & 1 & 0 & - & 0 & - & 0 & 0 & 0 \\
\hline Echinidae & 0 & 1 & 1 & 1 & 0 & 1 & 0 & - & 0 & - & 0 & 0 & 0 \\
\hline Echinometridae & 0 & 1 & 1 & 0 & 0 & 1 & 0 & - & 0 & - & 0 & 0 & 0 \\
\hline Strongylocentrotidae & 0 & 1 & 1 & $0 / 1$ & 0 & 1 & 0 & - & 0 & - & 0 & 0 & 0 \\
\hline Toxopneustidae & 0 & 1 & 1 & $0 / 1$ & 0 & 1 & 0 & - & 0 & - & 0 & 0 & 0 \\
\hline Echinoneidae & 0 & 0 & - & - & 0 & 1 & 1 & - & 0 & 0 & 1 & 0 & - \\
\hline Apatopygidae & 0 & 0 & - & - & 0 & 1 & 1 & - & 0 & 1 & 1 & 0 & - \\
\hline Cassidulidae & 0 & 0 & - & - & 0 & 1 & 2 & 0 & 0 & $?$ & 1 & 0 & - \\
\hline Echinolampadidae & 0 & 0 & - & - & 0 & 1 & 2 & 0 & 0 & $?$ & 1 & 0 & - \\
\hline Neolampadidae & 0 & 0 & - & - & 0 & 1 & 2 & 0 & 0 & $?$ & 1 & 0 & - \\
\hline Clypeasteridae & 0 & 0 & - & - & 0 & 1 & 2 & 1 & 0 & $?$ & 1 & 1 & - \\
\hline Arachnoididae & 0 & 0 & - & - & 0 & 1 & 2 & 1 & 0 & $?$ & 1 & 0 & - \\
\hline Laganidae & 0 & 0 & - & - & 0 & 0 & - & - & - & - & - & 0 & - \\
\hline Fibulariidae & 0 & 0 & - & - & 0 & 0 & - & - & - & - & - & 0 & - \\
\hline Rotulidae & 0 & 0 & - & - & 0 & 0 & - & - & - & - & - & 0 & - \\
\hline Echinarachniidae & 0 & 0 & - & - & 1 & 0 & - & - & - & - & - & 0 & - \\
\hline Dendrasteridae & 0 & 0 & - & - & 1 & 0 & - & - & - & - & - & 0 & - \\
\hline Astriclypeidae & 0 & 0 & - & - & 1 & 0 & - & - & - & - & - & 0 & - \\
\hline Mellitidae & 0 & 0 & - & - & 1 & 0 & - & - & - & - & - & 0 & - \\
\hline Corystidae & 1 & 0 & - & - & 0 & 1 & 1 & - & 1 & 1 & 1 & 0 & - \\
\hline Urechinidae & 1 & 0 & - & - & 0 & 1 & 1 & - & 1 & 1 & 1 & 0 & - \\
\hline Plexechinidae & 1 & 0 & - & - & 0 & 1 & 1 & - & 1 & 1 & 1 & 0 & - \\
\hline Pourtalesiidae & 1 & 0 & - & - & 0 & 1 & 1 & - & $0 / 1$ & 1 & 1 & 0 & - \\
\hline Aeropsidae & 1 & 0 & - & - & 0 & 0 & - & - & - & - & - & 0 & - \\
\hline Hemiasteridae & 1 & 0 & - & - & 0 & 1 & 1 & - & 0 & 1 & 1 & 0 & - \\
\hline Paleopneustidae & 1 & 0 & - & - & 0 & 1 & 1 & - & $?$ & $?$ & $?$ & 0 & - \\
\hline Prenasteridae & 1 & 0 & - & - & 0 & 1 & 1 & - & $?$ & $?$ & $?$ & 0 & - \\
\hline Schizasteridae & 1 & 0 & - & - & 0 & $0 / 1$ & $-/ 1$ & - & $-/ 0$ & $-/ 1$ & $-/ 1$ & 0 & - \\
\hline Brissidae & 1 & 0 & - & - & 0 & 1 & 1 & - & 1 & 1 & 1 & 0 & - \\
\hline
\end{tabular}


Table 4 Compilation of the primary morphological findings of this study related to the sea urchin anterior stomach in the form of a character matrix (Continued)

\begin{tabular}{lllllllllll}
\hline Brissopsidae & 1 & 0 & - & - & 0 & 1 & 1 & - & 1 & 1 \\
\hline Loveniidae & 1 & 0 & - & - & 0 & 1 & 1 & - & 1 \\
\hline Spatangidae & 1 & 0 & - & - & 0 & 1 & 1 & - & 1 & 1 \\
\hline Maretiidae & 1 & 0 & - & - & 0 & 1 & 1 & - & 1 & 1 \\
\hline Asterostomatidae & 1 & 0 & - & - & 0 & 1 & 1 & - & 1 \\
\hline
\end{tabular}

The results have been condensed in order to provide a general overview of the phylogenetically informative characters (13 in total) that could be derived from our analysis. See the "Phylogenetic implications" section of the "Discussion" for an explanation of the characters used. - = not applicable, ? = data not available.

homologize these structures, designating them from here on with the term "gastric caecum". This potential homology was also briefly mentioned by Koehler [9].

Of specific interest are those sea urchin taxa that lack a gastric caecum entirely or in which this structure can be found either in a reduced state or fused with adjoining structures. The single echinothurioid species so far known to lack the additional festoon as well as the gastric caecum is Asthenosoma ijimai, as depicted by Agassiz \& Clark [32]. Since this description deviates from that of other species within the Echinothuriidae, it might be possible that the conspicuous lateral outcrop of the intestine in Amb III (Figure 3G) could actually be a part of the anterior stomach. Although the morphology of the esophagus in Chaetodiadema pallidum (Figure 4D) closely resembles that in other diadematid species, the stomach resembles that of cidaroids with respect to festoon number (nine) and gastric caecum (absent) in Amb III. Since this observation diverges from that in other diadematids, it needs to be verified in additional material. The digestive tract in arbacioids and salenioids is unlike those of most other "regular" sea urchin species because of its largely un-festooned, flat aspect that resembles the situation found in irregular sea urchins. Although it is difficult to differentiate individual festoons and the gastric caecum macroscopically in the Arbacioida and Salenioida, mesenterial strands connecting the digestive tract with the test clearly reveal the presence of these structures.

The apparent lack of a gastric caecum in the derived Clypeasteroida (Laganidae onwards, see Figure 2) is notable. Koehler [63] also mentioned the absence of such a structure in the derived clypeasteroids that he had termed the "glande intestinale" based on his analyses of the Clypeasteridae and Arachnoididae. However, he rejected a potential homology between this structure and the gastric caecum which he had previously observed in spatangoid taxa. Based on our data, we do not follow Koehler and instead consider the gastric caecum to be present in at least the two basal-most extant clypeasteroid taxa, the Clypeasteridae and the Arachnoididae. Whether the frilled zone (or sacculated area) at the abaxial edge of most parts of the stomach in certain derived clypeasteroid taxa must be considered a derivative of the gastric caecum needs to be assessed using comparative histological analyses on freshly fixed specimens. Our preliminary analyses using museum specimens were not successful due to the state of the material. Histological or even ultrastructural inferences might also be needed to determine whether the gastric caecum could have been internalized in taxa that lack both the gastric caecum as well as the sacculated area such as the Laganidae, the Fibulariidae, and the Rotulidae. Two further taxa within the Irregularia in which a gastric caecum appears to be absent are the two spatangoid genera Aeropsis and Aceste. No histological data are currently available for these taxa, and unfortunately not much is known regarding the biology of these deepsea echinoids. So far, these two genera are the only known to lack the prominent gastric caecum that seems to be a characteristic feature of all other members of the Atelostomata.

\section{(ii) Shape and size of the gastric caecum}

The gastric caecum observed in the "regular" as well as the irregular Euechinoidea varies greatly in shape and size. In "regular" species, this structure is most prominent in taxa such as the Echinothurioida (e.g. Figure $3 \mathrm{D}$ ) or the Diadematidae (e.g. Figure 4C). In the latter taxon, its orientation has additionally shifted from a lateral towards an adaxial position. However, a large gastric caecum can also be found in basal echinacean taxa such as Glyptocidaris crenularis (Figure 4E) and Stomopneustes variolaris (Figure 4F), its position lateral to the additional festoon in Amb III resembling the situation in the Echinothurioida rather than that in the Diadematidae. A small lateral dilation may be present in the Cidaroida as well, but based on the above mentioned homology hypothesis, we rule out any evolutionary relationship of this structure with the gastric caecum present in the Euechinoidea. Whether the cidaroid dilation has to be seen as the precursor (or the successor) of the additional festoon in Amb III encountered in "regular" euechinoids is impossible to determine based on the currently available data alone.

Although a gastric caecum is present in the more derived taxa of the Echinacea such as the Arbacioida, 
the Salenioida, and the Temnopleuroida, its exact size and shape are difficult to determine because it is largely fused with the adjoining additional festoon in Amb III. The distributional patterns of shape and size of the gastric caecum are more complex within the taxon Echinoida. Here, in the Parasaleniidae, Echinometridae, Strongylocentrotidae, and in part also the Toxopneustidae, the morphology of the anterior stomach closely resembles that found in Stomopneustes variolaris (Figures 4, 5, and 9). In contrast, the anterior stomach in the Parechinidae, Echinidae, and in part also in the Toxopneustidae is characterized by a gastric caecum that is largely fused with the additional festoon in Amb III, and which therefore more closely resembles the situation encountered in the Temnopleuroida.

A rather common feature of the gastric caecum in the Irregularia is its position at the outer edge of the anterior stomach. However, despite this trait, the greatest variability in size and shape of the gastric caecum can be observed in irregular sea urchins. Here, the gastric caecum can either be absent (as in the derived Clypeasteroida and certain Spatangoida) or present in the form of multiple small nodules (basal Clypeasteroida), as multiple finger-like pouches (certain Cassiduloida), as well as in the form of a single smooth or lobate pouch that may extend from about a third (e.g. Echinoneus, Figure 9L) to more than half of the specimen's entire test length (e.g. Abatus, Figure 9T). In addition, the connections of the gastric caecum to the anterior stomach may be either through a broad canal or through a slit-like opening which may aid in preventing sediment grains from entering - the gastric caecum of the irregular sea urchins observed in this study always had a liquid content (Figure $11 \mathrm{H}-\mathrm{L}$ ). Furthermore, the macroscopic appearance of the gastric caecum in most Irregularia is always that of a thin-walled, semi-transparent pouch which in most cases easily permits distinction of this part of the stomach.

Interestingly, the description by Agassiz [13] of the gastric caecum in Metalia sternalis, a derived spatangoid, closely resembles that of the gastric caecum found in cassiduloids. Since we have never found a similarly shaped gastric caecum in any other spatangoid, we presume that Agassiz must have erred when determining the species. Although Koehler [9] noted differences in the extent to which the walls of the gastric caecum are covered in folds within spatangoids, we were not able to identify any meaningful pattern within this taxon. However, the gastric caecum in the Cassidulidae, Echinolampadidae, Neolampadidae, basal Clypeasteroida, as well as certain Holasteroida is smooth-walled; we consider this condition to be a derived feature, based on the presence of a lobate gastric caecum in the more basal irregular taxa Echinoneidae (Figure 9L) and Apatopygidae (Figure 6B).
Analyses of different developmental stages of specimens from five species, Paracentrotus lividus, Psammechinus miliaris, Echinometra viridis, Echinoneus cyclostomus and Arachnoides placenta reveal that the additional festoon as well as the gastric caecum are present already in juvenile specimens. The observed distinct architecture of the anterior stomach must therefore be considered as a development-independent feature.

\section{(iii) Mesenterial suspension of the anterior stomach}

Of particular interest for the elucidation of gastric caecum homologies are the various mesenteries that are involved in the suspension of the anterior stomach. The abaxial edge of the entire stomach is usually attached to the test through a more or less continuous mesenterial sheet that also follows the vertical inflections of the festoons, in turn permitting to identify individual festoons using primarily MRI. A second mesentery, termed the dorso-ventral mesentery, attaches the esophagus to the test as well as to the axial complex [31]. The gastric caecum is partly surrounded by and, therefore, included within this mesentery. Except for the Cidaroida (which do not possess the additional festoon in Amb III as well as the gastric caecum) and atelostomates (detailed below), the dorso-ventral mesentery extends from the central oral-aboral axis towards the test near the border of IAmb 2 and Amb III (Figure 11B-J). In the more derived Spatangoida (Brissidae onwards, see Figure 2), this connection has shifted counter-clockwise at least as far as IAmb 3, resulting in an oblique form of the entire gastric caecum (Figure 11K, L) as opposed to a straight form seen in most other irregular sea urchins (Figure $11 \mathrm{H}-\mathrm{J})$. A similar observation can be made in certain holasteroid species, especially in Corystus (Figure 8A) and Urechinus (Figure 8C).

Further suspension of the anterior stomach can be observed in the Diadematidae, where the "ends" of the lower gut loop (i.e. the stomach) are fused by a strong mesentery (Figure 4A). A similar - clearly convergent development can be observed in the Clypeasteridae (Figure $6 \mathrm{E})$.

\section{(iv) Integration of the anterior stomach into the digestive tract haemal system}

Sea urchins are characterized by a relatively complex haemal system $[9,22,33,43]$. The stomach is well integrated into this system and is supplied by an inner as well as outer marginal haemal duct. The inner marginal haemal duct ascends from the esophageal haemal ring along the esophagus and borders the adaxial edge of the anterior stomach (Figure 12A). The outer marginal haemal duct borders the abaxial edge of the anterior stomach and sends out branches towards the dorso-ventral mesentery which is connected to the axial complex and its haemal spaces. The outer marginal haemal duct is present in this form presumably in all "regular" sea 


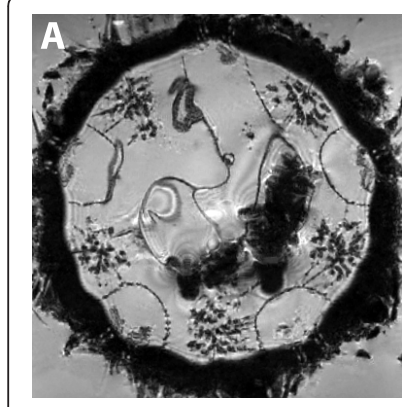

Cidaris cidaris

(NHM 1925.10.30.10.3-113)

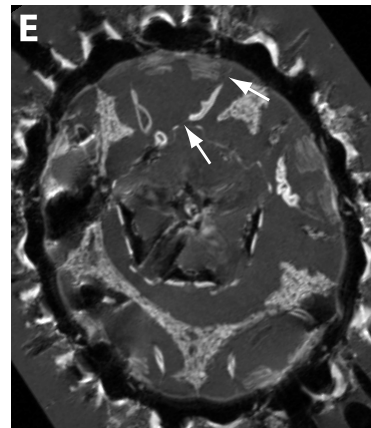

Parasalenia gratiosa

(NHM 1983.2.15.7)

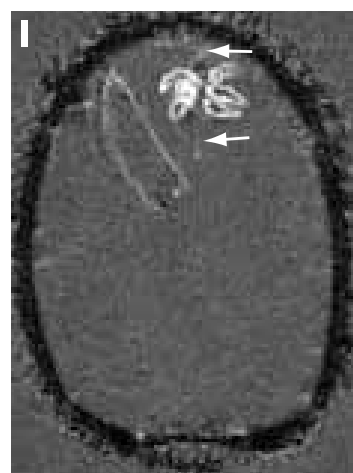

Cassidulus cariboearum (CASIZ 112632)

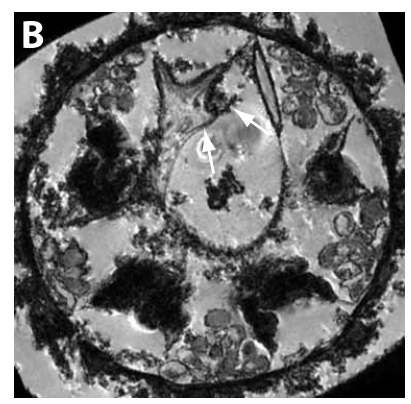

Aspidodiadema hawaiiense (USNM 27590)

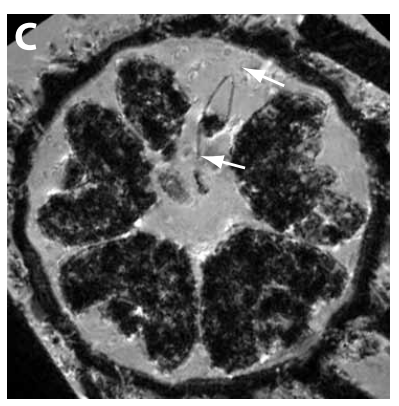

Stomopneustes variolaris (USNM E45930)

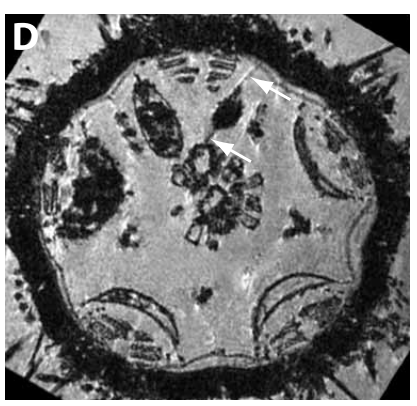

Mespilia globulus (ZMB 5620)

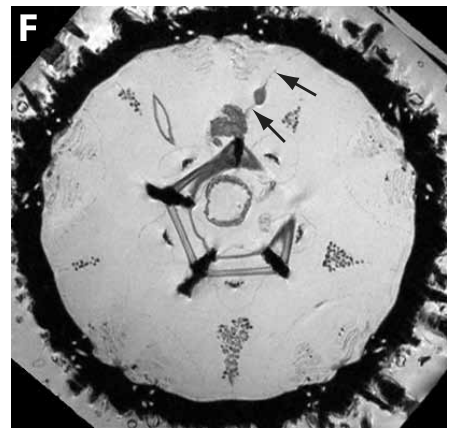

Psammechinus miliaris (this study)

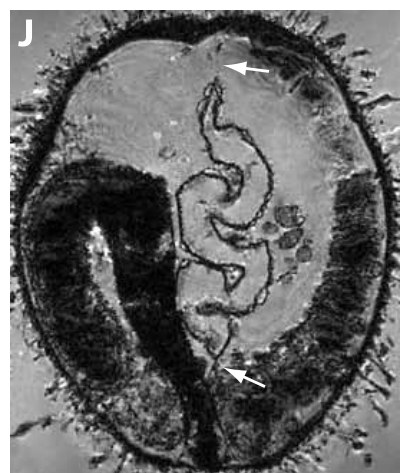

Abatus cavernosus

(ZMB 5854)

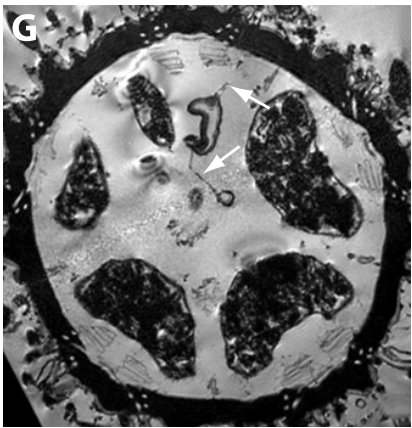

Strongylocentrotus purpuratus (CASIZ 5724)

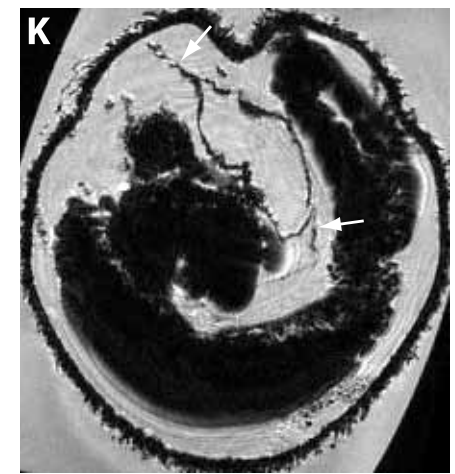

Echinocardium cordatum (this study)

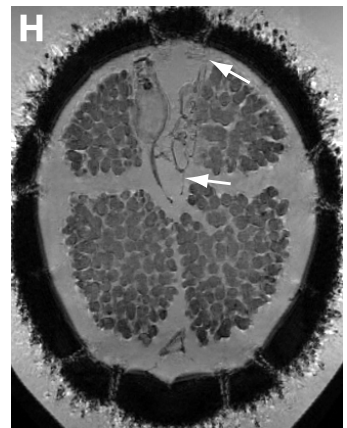

Echinoneus cyclostomus (NHM 1969.5.1.105)

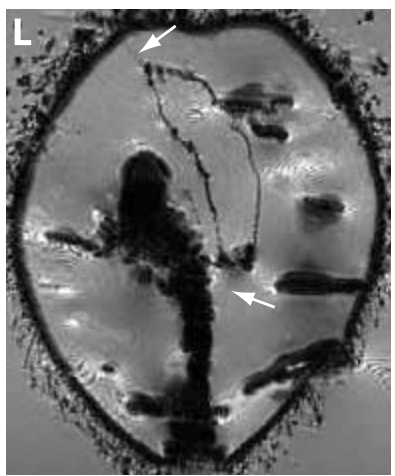

Spatangus purpureus

(this study)

Figure 11 Homology of the sea urchin gastric caecum based on its integration into the mesenterial system, in particularl the dorsoventral mesentery, as a primary criterion. Virtual horizontal sections based on MRI scans of Cidaridae (A), Aspidodiadematidae (B),

Stomopneustidae (C), Temnopleuridae (D), Parasaleniidae (E), Parechinidae (F), Strongylocentrotidae (G), Echinoneidae (H), Cassidulidae (I),

Schizasteridae (J), Loveniidae (K), and Spatangidae (L). The gastric caecum - where present - is attached to esophagus, axial complex, and the test through the dorso-ventral mesentery (arrows). In the Cidaroida (A), the dorso-ventral mesentery attaches to the single festoon present in ambulacrum III. In the more derived spatangoid and certain holasteroid taxa, this mesentery is shifted away from its original insertion near ambulacrum III towards interambulacrum 3, resulting in an oblique position of the gastric caecum (K, L). Note that the gastric caecum, in contrast to the rest of the digestive tract, is always free of sediment grains in the burrowing irregular taxa (H-L). Not to scale.

urchins (Figure 12B). In the Spatangoida, the outer marginal haemal duct is located on the aboral side of the gastric caecum (Figure 12C). The inner marginal haemal duct, however, underwent considerable changes, although the precise timing of these events is difficult to trace based on the present data alone. These changes have led to the evolution of a side-branch of the inner marginal haemal duct which descends from the main branch in order to supply the adoral side of the gastric caecum. Such a novel side-branch can be found in the Clypeasteroida (Figure 6E, F) as well as the Spatangoida (Figure 12D). We were not able to identify this structure in the Echinoneoida, the Cassiduloida, or the Holasteroida and are therefore unsure about its origins. As indicated above, a number of authors elaborated on the complex anatomy of the sea urchin haemal system and 


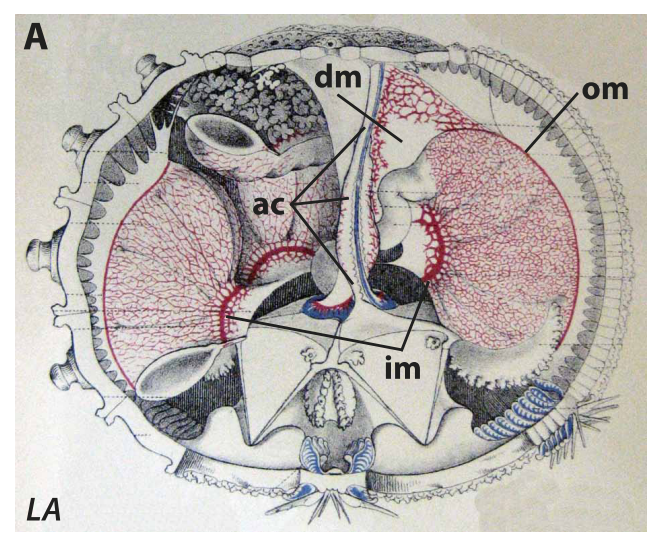

Cidaris cidaris (from [33])

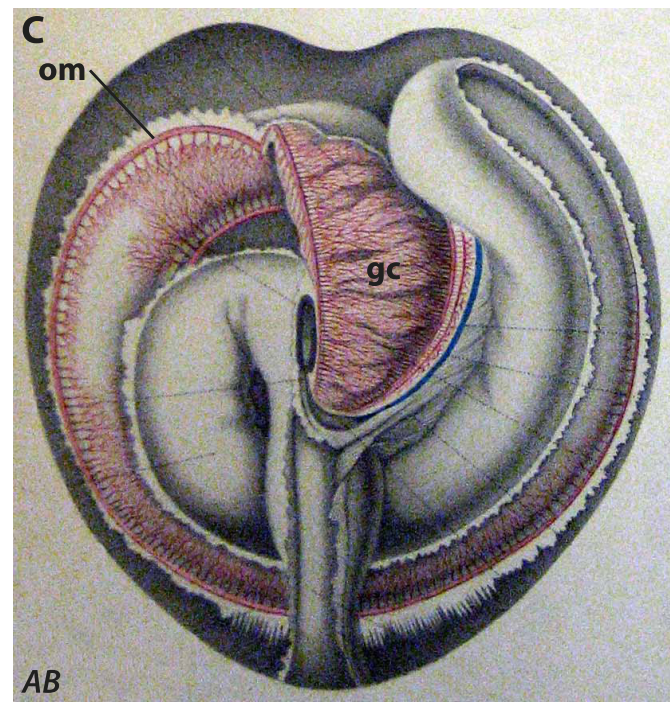

Spatangus purpureus (from [9])

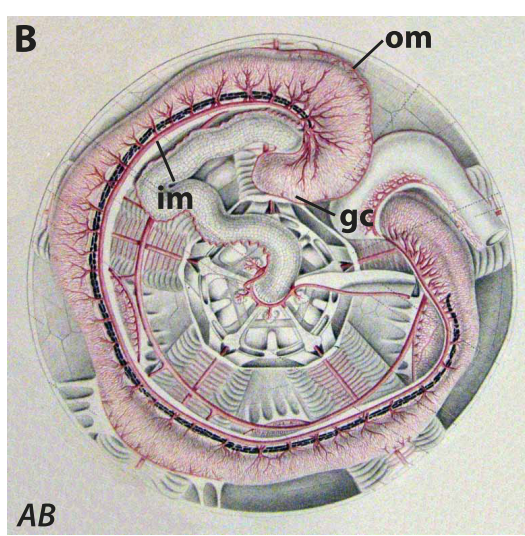

Echinus esculentus (from [43])

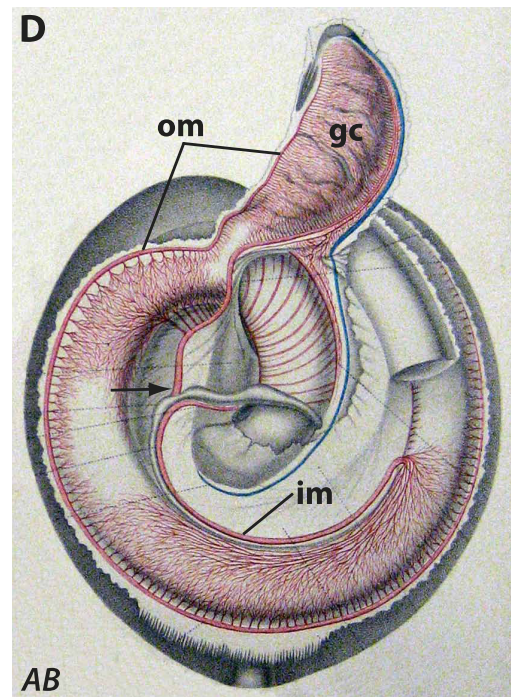

Spatangus purpureus (from [9])

Figure 12 Homology of the sea urchin gastric caecum based on its integration into the haemal system of the digestive tract as a primary criterion. Schematic representations of the digestive tract haemal system in Cidaridae (A), Echinidae (B), and Spatangidae (C, D). The sea urchin stomach is accompanied by an inner (im) as well as an outer (om) marginal haemal duct. The outer marginal haemal duct sends out branches towards the dorso-ventral mesentery ( $\mathrm{dm}$ ) which is connected to the axial complex (ac) (A). The gastric caecum (gc) - if present (B-D) is well-integrated into the haemal system. The black arrow in (D) depicts the conspicuous side branch of the inner marginal haemal duct present in presumably all spatangoids and potentially further irregular sea urchin taxa. $A B=$ aboral view, $L A=$ lateral view. Not to scale.

so far the Cidaroida [33], the Echinothurioida [34], the Arbacioida [22], the Echinoida [22,23,43,48], the Temnopleuroida [41], the Clypeasteroida [40,55], and the Spatangoida $[8,9,12,64,66,69]$ have been covered. Since the present study did not reveal any novel data on this subject, we will not consider the haemal system of the digestive tract any further.

\section{Evolutionary origin of the highly specialized gastric caecum}

As detailed in the previous section, we believe the gastric caecum found in most irregular sea urchins to be homologous with the dilation of the anterior stomach observed in the "regular" Euechinoidea. This dilation can therefore be seen as the precursor of the spatangoid gastric caecum, proposing a solution for the question of the evolutionary origin of this enigmatic organ.

If we consider the presence of the additional festoon as well as the gastric caecum in Amb III as a synapomorphy of the Euechinoidea, in turn the question of the origin of these structures arises. Since the extant sister group to the Euechinoidea, the Cidaroida, possesses only a single festoon in Amb III, we are not able to determine precisely what the condition in the ancestor of both taxa might have been. To clarify this aspect, analyses of mesenterial strand imprints upon the internal part of the test, potentially visible in extant and well-preserved fossil specimens (sensu Roman [73]), might be helpful. It seems obvious to assume that the ancestor of both Cidaroida and Euechinoidea should 
have possessed two festoons in Amb III, given the otherwise strong pentameric arrangement of the internal anatomy of "regular" sea urchins. This notion is supported in part by the comparatively large anterior stomach in two basal cidaroid taxa, the Histocidaridae and the Ctenocidaridae, which could hint at a stepwise reduction of the additional festoon within the Cidaroida. But then the question arises as to why the Cidaroida had lost this part of the anterior stomach, since there is no structure occupying the void between the first (in Amb III) and the last (in Amb II) festoon of the stomach that can be found in all cidaroids - Stewart's organs as well as large gonads are present in the basal Euechinoidea as well. Further studies on the general homology of mesenteries in sea urchins might help to answer this question.

A probable explanation for the presence of additional digestive tract elements in the basal Euechinoidea such as the Echinothurioida or Diadematidae might be that their feeding habits differ from those of the Cidaroida. Most "regular" sea urchins are considered omnivorous, although cidaroids display a certain degree of carnivory, whereas most other "regular" echinoids are considered omnivorous with herbivorous habits [5]. In addition, the "regular" euechinoids are capable of forming so-called food pellets, primarily using their buccal cavity and pharynx. To what degree the additional festoon as well as the gastric caecum might play a role in processing mucous-enclosed food pellets needs to be addressed in future studies. One might hypothesize that water circulation throughout the digestive tract is facilitated when the gut content is packed into pellets which in turn could contribute to the feeding process and its dynamics. Extended comparative histological analyses will be necessary to elucidate any potential usefulness of the gastric caecum in "regular" euechinoids with regard to feeding or nutrient absorption.

Once present, the gastric caecum was modified during evolution, with a number of significant changes in "regular" and irregular sea urchins becoming apparent: (i) reduction of stomach festoons and fusion of the additional festoon with the lateral dilation (Arbacioida, Salenioida); (ii) fusion of the additional festoon with the lateral dilation (Temnopleuroida, some Echinoida); (iii) specialization of the lateral dilation into a thin-walled, fluid-filled, semitransparent pouch always free of sediment (Irregularia); (iv) division of the single pouch into numerous finger-like sacs and further reduction into grape-like nodules (some Cassiduloida and Clypeasteroida); (v) enlargement of the pouch combined with a shift of the opening towards the anterior (some Cassiduloida, Atelostomata), and finally (vi) shifting of the axis of orientation of the pouch from an axis along Amb III-IAmb 5 to IAmb 3-Amb I (some Holasteroida and Spatangoida).
The drastic changes observed within the Irregularia, including the wholesale reduction of the gastric caecum in some taxa, presumably correlate with feeding habits and lifestyle of the various taxa. Although the precise function of the gastric caecum remains unknown, it seems obvious that a role in the digestive process can be assumed. Irregular sea urchins are primarily infaunal deposit feeders and are known to inhabit various types of sediment [74]. Future studies will therefore have to address the question to what extent the shape and size of gastric caeca might correlate with sediment type and feeding habits.

\section{Phylogenetic implications}

From our anatomical descriptions it becomes clear that the absence or presence as well as shape and relative size of the gastric caecum characterizes individual sea urchin taxa. The compilation of our findings presented here suggests a number of phylogenetic conclusions. This includes support for the monophyly of the Cidaroida, the Euechinoidea, as well as the Irregularia. The divergent shapes of the gastric caecum in cassiduloid families suggest a potential non-monophyly of the Cassiduloida, a conclusion reached by others (e.g. [75,76]). The loss of a gastric caecum could furthermore support the monophyly of the currently unnamed taxon comprising the Laganina and the Scutellina, with additional support for the Scutellina being obtained through the presence of a conspicuously frilled zone at the abaxial edge of most parts of the stomach. Loss of the gastric caecum in the spatangoid genera Aeropsis and Aceste could be indicative of the need to group these taxa more closely together. The presence of a flattened stomach without festoons is in support of a potentially close relationship between the Salenioida and the Irregularia. The morphology of the anterior stomach in sea urchins provides a number of characters with phylogenetic signal (see Table 4 for a character matrix):

1. Anterior stomach extends into ambulacra I and II: absent (0) (Figure 3A); present (1) (Figure 8G).

2. Stomach festoons: absent (0) (Figure 9L); present (1) (Figure 9C).

3. Number of festoons in ambulacrum III: one (0) (Figure 3C); two (1) (Figure 3D).

4. Festoons in ambulacrum III: separate (0) (Figure 4F); fused (1) (Figure 4G).

5. Stomach sacculated at abaxial edge: absent (0) (Figure 7C); present (1) (Figure 7L).

6. Gastric caecum: absent (0) (Figure 3B); present (1) (Figure 3E).

7. Shape of gastric caecum: dilation (0) (Figure $5 \mathrm{H}$ ); single pouch (1) (Figure 6A); multiple pouches (2) (Figure $6 \mathrm{C})$.

8. Shape of multiple pouches: finger-like (0) (Figure $6 \mathrm{C})$; grape-shaped (1) (Figure 6E). 
9. Mesenterial suspension of gastric caecum: along axis from ambulacrum III to interambulacrum 5 (0) (Figure 11J); along axis from interambulacrum 3 to ambulacrum I (1) (Figure 11K).

10. Position of connection(s) between gastric caecum and anterior stomach with regard to the gastric caecum: median (0) (Figure 6A); anteriorly (1) (Figure 8I).

11. Macroscopic appearance of gastric caecum: undifferentiated from stomach (0) (Figure 5B); thin-walled, semi-transparent (1) (Figure 8M).

12. Stomach ends fused in ambulacrum III: absent (0) (Figure 4E); present (1) (Figure 4A).

13. Position of dilation in ambulacrum III: lateral (0) (Figure 4E); adaxial (1) (Figure 4A)

\section{Conclusions}

The present study constitutes a contribution towards better understanding of digestive tract structures in sea urchins from an evolutionary perspective. Our data permit identification of the relevant transformational stages in the course of the evolution of the highly specialized gastric caecum present in the derived Spatangoida. According to our findings, the sea urchin gastric caecum constitutes a synapomorphy of the Euechinoidea. Its occurrence in "regular" euechinoids is linked to the presence of an additional festoon of the anterior stomach in Amb III. Both structures, the additional festoon and the gastric caecum, are absent in the Cidaroida. Since the degree of specialization of the gastric caecum is most pronounced in the predominantly sediment-burrowing irregular taxa, we hypothesize that its evolution is closely linked to the development of more elaborate infaunal lifestyles. We provide a comprehensive study of the origin and evolutionary plasticity of a conspicuous digestive tract structure, the gastric caecum, in a major taxon of the extant invertebrate macrozoobenthos.

\section{Methods}

\section{Species used in this study}

The specimens referred to in this study are listed in Tables 2 and 3 together with information on the systematic classification of each species, the source of the data used in the study, specimen ID where applicable, and literature references. The freshly fixed specimens were collected in Elba (Italy), Heligoland (Germany), Sydney Harbour (Australia), Discovery Bay (Jamaica), New Caledonia (France) or were purchased from aquarium supply companies. The systematic classifications are based upon results obtained by [75-84].

\section{Dissection and photography}

Dissection was performed on freshly fixed as well as museum specimens under direct observation through a stereo-microscope equipped with a digital camera for documentation.

\section{Magnetic resonance imaging}

Magnetic resonance imaging was performed using the methods described in $[25,85,86]$. Imaging was carried out in Berlin and Würzburg, Germany using high-field small animal MRI scanners equipped with $7 \mathrm{~T}, 9.4 \mathrm{~T}$, and 17.6 $\mathrm{T}$ super-conducting electromagnets, respectively. The resolution of the datasets acquired varied between $\sim(20 \mu \mathrm{m})^{3}$ and $(96 \mu \mathrm{m})^{3}$ for 3D protocols and was $50 \times 50 \times 200 \mu \mathrm{m}^{3}$ or $78 \times 78 \times 300 \mu^{3}$ for the $2 \mathrm{D}$ protocols employed. Tables 2 and 3 list the resolutions achieved for every species analyzed by MRI. Image processing was carried out using ImageJ $1.42 \mathrm{q}(\mathrm{NIH}$, USA) and its Volume Viewer plugin. Unfortunately, we are currently unable to provide the raw image data online due to the lack of a centralized repository for digital morphological data (see [87] for discussion).

\section{D modelling and visualization}

3D image reconstruction and modelling were performed using the methods described in $[25,85,86]$. The interactive 3D models in Figure 10 were embedded using the Adobe 3D Reviewer software (part of the Adobe Acrobat 9 Pro Extended suite) according to procedures described in [88-90]. All figures were arranged and assembled using Adobe Photoshop CS3 and Adobe Illustrator CS3.

\section{List of abbreviations used}

2D: two-dimensional; 3D: three-dimensional; AAD: Australian Antarctic Division, Kingston, Australia; AM: Australian Museum, Sydney, Australia; CASIZ: California Academy of Sciences Invertebrate Zoology, San Francisco, USA; MNHN: Muséum Nationale de la Histoire Naturelle, Paris, France; MRI: magnetic resonance imaging; NHM: Natural History Museum, London, United Kingdom; NHMW: Naturhistorisches Museum, Wien, Austria; NIWA: National Institute of Water \& Atmospheric Research, Wellington, New Zealand; USNM: National Museum of Natural History, Washington D.C., USA; ZMB: Systematische Zoologie am Museum für Naturkunde, Berlin, Germany; ZMH: Zoologisches Institut und Museum, Hamburg, Germany; ZSM: Zoologische Staatssammlung, München, Germany.

\section{Acknowledgements}

The authors thank Susanne Mueller (Charité-Universitätsmedizin, Berlin, Germany), Cornelius Faber (Universitätsklinikum Münster, Germany), and Leif Schröder (Leibniz-Institut für Molekulare Pharmakologie, Berlin, Germany) for continued MRI support. We are grateful to Nadia Améziane (MNHN), Owen Anderson (NIWA), Andrew Cabrinovic (NHM), Danny Eibye-Jacobsen (ZMK), Ty Hibberd (AAD), Stephen Keable (AM), Carsten Lüter (ZMB), Andreas Kroh (NHMW), Claus Nielsen (ZMK), David Pawson (USNM), Bernhard

Ruthensteiner (ZSM), Andreas Schmidt-Rhaesa (ZMH), and Andrew Smith (NHM) for generous supply of sea urchin museum specimens. We also thank Peter Jumars (Darling Marine Center, Walpole, USA) for granting access to unpublished data on Brisaster latifrons gathered by Stephen B. Sampson. We are grateful to Maria Byrne (University of Sydney, Australia) and Ashley Miskelly (Blackheath, Australia) for introducing one of us (AZ) to the Australian echinoid fauna. We would also like to thank Saloua M'Zoudi (Université Libre de Bruxelles, Belgium) for technical assistance with histological analyses. Comments from two anonymous reviewers helped to improve the manuscript. We are grateful to Andreas Ziegler (Charité- 
Universitätsmedizin, Berlin, Germany) for critical reading of the manuscript. This article is a contribution of the Centre Interuniversitaire de Biologie Marine (CIBIM).

\section{Author details}

${ }^{1}$ Institut für Immungenetik, Charité-Universitätsmedizin Berlin, Thielallee 73, 14195 Berlin, Germany. ${ }^{2}$ California Academy of Sciences, Golden Gate Park, 55 Music Concourse Drive, San Francisco, California, 94118, USA. ${ }^{3}$ Laboratoire de Biologie Marine, Université Libre de Bruxelles, 50 avenue F.D. Roosevelt, 1050 Bruxelles, Belgium.

\section{Authors' contributions}

AZ designed and coordinated the study, carried out dissections and specimen photography, performed literature search, prepared specimens for MRI scanning, scanned specimens, carried out 3D modelling, and wrote the manuscript. RM carried out dissections and specimen photography. GR performed dissection as well as literature search. CD carried out dissection, literature search and histological analyses. CD and RM contributed to writing the manuscript. All authors read and approved of the final version.

\section{Competing interests}

The authors declare that they have no competing interests.

Received: 8 July 2010 Accepted: 18 October 2010

Published: 18 October 2010

\section{References}

1. Schmidt-Rhaesa A: Intestinal systems. In The evolution of organ systems. Edited by: Schmidt-Rhaesa A. Oxford: Oxford University Press; 2007:218-239.

2. Moyes CD, Schulte PM: Principles of animal physiology New York: Benjamin Cummings Publishing Company 2008.

3. Fankboner PV: Digestive systems of invertebrates. Encyclopedia of Life Sciences Chichester: John Wiley \& Sons Ltd 2002

4. Holland ND, Ghiselin MT: A comparative study of gut mucous cells in thirty-seven species of the class Echinoidea (Echinodermata). Biological Bulletin 1970, 138:286-305.

5. De Ridder C, Jangoux M: Digestive systems: Echinoidea. In Echinoderm nutrition. Edited by: Jangoux M, Lawrence JM. Rotterdam: A.A. Balkema; 1982:213-234.

6. Jensen M: Digestive system of echinoids. In Echinoderms through time. Edited by: David B, Guille A, Féral JP, Roux M. Rotterdam: A.A. Balkema; 1994:731.

7. De Ridder C, Lawrence JM: Food and feeding mechanisms: Echinoidea. In Echinoderm nutrition. Edited by: Jangoux M, Lawrence JM. Rotterdam: A.A. Balkema; 1982:57-115.

8. Hoffmann CK: Zur Anatomie der Echinen und Spatangen. Niederländisches Archiv für Zoologie 1871, 1:11-112.

9. Koehler R: Recherches sur les échinides des côtes de Provence. Annales du Musée d'Histoire Naturelle Marseille - Zoologie 1883, 1(Suppl 3):1-167.

10. Lawrence JM: A functional biology of echinoderms Baltimore: Johns Hopkins University Press 1987.

11. De Ridder C: La nutrition chez les échinodermes psammivores. Étude particulière du spatangide fouisseur, Echinocardium cordatum (Pennant) (Echinodermata, Echinoidea). PhD thesis Université Libre de Bruxelles 1986.

12. De Ridder $C$, Jangoux $M$ : The digestive tract of the spatangoid echinoid Echinocardium cordatum (Echinodermata): morphofunctional study. Acta Zoologica (Stockholm) 1993, 74:337-351.

13. Agassiz A: Revision of the Echini. Illustrated Catalogue of the Museum of Comparative Zoology 1872, 7.

14. Cuénot L: Anatomie, éthologie et systématique des échinodermes. In Traité de Zoologie, Volume 11. Edited by: Grassé PP. Paris: Masson; 1948:1-363.

15. Thorsen MS: Microbial activity, oxygen status and fermentation in the gut of the irregular sea urchin Echinocardium cordatum (Spatangoida: Echinodermata). Marine Biology 1998, 132:423-433.

16. Thorsen MS: Abundance and biomass of the gut-living microorganisms (bacteria, protozoa and fungi) in the irregular sea urchin Echinocardium cordatum (Spatangoida: Echinodermata). Marine Biology 1999, 133:353-360.

17. Henry V: Étude des ferments digestifs chez quelques invertébrés. Comptes rendus de la Société de Biologie 1903, 55:1316-1318.
18. Ludwig H, Hamann O: Die Seeigel. Dr. H. G. Bronn's Klassen und Ordnungen des Thier-Reichs. Band 2, Abtheilung 3, Buch 4 Leipzig: C.F. Winter'sche Verlagshandlung 1904.

19. Saucède T, Mooi R, David B: Phylogeny and origin of Jurassic irregular echinoids (Echinodermata: Echinoidea). Geological Magazine 2007, 144:333-359.

20. Tiedemann F: Anatomie der Röhren-Holothurie (Holothuria tubulosa), des Pomerantz-farbigen Seesterns (Astropecten aurantiacus) und des Stein-Seeigels (Echinus saxatilis) Landshut: Joseph Thomannsche Buchdruckerei, Verlag der Landshuter Zeitung 1816.

21. Valentin GG: Anatomie du genre Echinus. In Livraison des Monographies d' Échinodermes vivants et fossiles. Edited by: Agassiz L. Neuchâtel: Petitpierre; 1841:1-126.

22. Bonnet $A$ : Recherches sur l'appareil digestif et absorbant de quelques échinides réguliers. Annales de l'Institut Océanographique 1925, 2:209-228.

23. McRae A: Evechinus chloroticus (Val.), an endemic New Zealand echinoid. Transactions of the Royal Society of New Zealand 1959, 86:205-267.

24. Lewis JB: Feeding and digestion in the tropical sea urchin Diadema antillarum Philippi. Canadian Journal of Zoology 1964, 42:549-557.

25. Ziegler A, Faber C, Mueller S, Bartolomaeus T: Systematic comparison and reconstruction of sea urchin (Echinoidea) internal anatomy: a novel approach using magnetic resonance imaging. BMC Biology 2008, 6:33.

26. Holland ND, Lauritis JA: The fine structure of the gastric exocrine cells of the purple sea urchin, Strongylocentrotus purpuratus. Transactions of the American Microscopical Society 1968, 87:201-209.

27. Powis de Tenbossche $\mathrm{T}$ : Comportement alimentaire et structures digestives de Paracentrotus lividus (Lamarck) (EchinodermataEchinoidea). Diploma thesis. Université Libre de Bruxelles 1978.

28. Holland ND, Ghiselin MT: Magnetic resonance imaging (MRI) has failed to distinguish between smaller gut regions and larger haemal sinuses in sea urchins (Echinodermata: Echinoidea). BMC Biology 2009, 7:39.

29. Lovén S: Echinologica. Bihang till Koniglika Svenska Vetenskaps-Akademiens Handlingar 1892, 18:Suppl 4.

30. Ziegler $A$ : Non-invasive imaging and $3 D$ visualization techniques for the study of sea urchin internal anatomy. PhD thesis Freie Universität Berlin 2008.

31. Ziegler A, Faber C, Bartolomaeus T: Comparative morphology of the axial complex and interdependence of internal organ systems in sea urchins (Echinodermata: Echinoidea). Frontiers in Zoology 2009, 6:10.

32. Agassiz A, Clark HL: Hawaiian and other Pacific Echini. The Salenidae, Arbaciadae, Aspidodiadematidae, and Diadematidae. Memoirs of the Museum of Comparative Zoology 1908, 34:43-132.

33. Prouho $\mathrm{H}$ : Recherches sur le Dorocidaris papillata et quelques autres échinides de la Méditerranée. Archives de Zoologie Expérimentale et Générale 1887, 5:213-380.

34. Schurig W: Anatomie der Echinothuriden. In Wissenschaftliche Ergebnisse der deutschen Tiefsee-Expedition auf dem Dampfer "Valdivia" 1898-1899. Fünfter Band, dritte Lieferung. Edited by: Chun C. Jena: Fischer; 1906:

35. Agassiz A, Clark HL: Hawaiian and other Pacific Echini. The Echinothuridae. Memoirs of the Museum of Comparative Zoology 1909, 34:135-203.

36. Agassiz A: Report on the Echinoidea, dredged by H.M.S. Challenger during the years 1873-1876. In Report on the scientific results of the voyage of H.M.S. Challenger during the years 1873-76. Edited by: Wyville Thomson C. London: Longman 1881:.

37. Clark HL: Hawaiian and other Pacific Echini. The Pedinidae, Phymosomatidae, Stomopneustidae, Echinidae, Temnopleuridae, Strongylocentrotidae, and Echinometridae. Memoirs of the Museum of Comparative Zoology 1912, 34:205-383.

38. Mortensen T: III. 1. Aulodonta. A Monograph of the Echinoidea Copenhagen: C. A. Reitzel 1940.

39. Campos LS, Moura RB: Macrostructure and evolution of the digestive system in Echinoida (Echinodermata). Zoomorphology 2008, 127:135-141.

40. Coe WR: Echinoderms of Connecticut. Connecticut State Geological and Natural History Survey Bulletin 1912, 19:1-152.

41. Aiyar RG: Salmacis - The Indian sea urchin. In The Indian Zoological Memoirs on Indian Animal Types. Edited by: Bahl KN. Lucknow: Lucknow Publishing House; 1938:7.

42. Ziegler A, Angenstein F: Analyse von Seeigeln (Echinoidea) mit Hilfe der bildgebenden Magnetresonanztomographie. Mikrokosmos 2007, 96:49-54. 
43. Perrier E: Recherches sur l'appareil circulatoire des oursins. Archives de Zooloogie Experimentale et Générale 1875, 4:605-643.

44. Chadwick HC: Echinus. Liverpool Marine Biology Committee Memoir III Liverpool: University Press 1900.

45. MacBride EW: Echinodermata. The Cambridge Natural History London: Macmillan 1906, 427-623.

46. Kükenthal WG: Leitfaden für das zoologische Praktikum. 6. Auflage Jena: Gustav Fischer 1912.

47. Fox R: Strongylocentrotus droebachiensis. Green sea urchin. Invertebrate Anatomy OnLine 2001 [http://webs.lander.edu/rsfox/invertebrates/ strongylocentrotus.html].

48. Strenger A: Sphaerechinus granularis (Violetter Seeigel), Anleitung zur makroskopischen und mikroskopischen Untersuchung. In Großes Zoologisches Praktikum, Band 18. Edited by: Siewing R. Stuttgart: Fischer; 1973:1-68

49. Hyman LH: IV. Echinodermata. The Invertebrates New York: McGraw-Hill Book Co 1955, 413-588.

50. Westergren AM: Echinoneus and Micropetalon. Echini. Reports on the scientific results of the expedition "Albatross" Memoirs of the Museum of Comparative Zoology 1911, 39(2).

51. Koehler R: Échinides du Musée Indien à Calcutta. II. Clypeasteridés et Cassidulidés. Echinoderma of the Indian Museum, Part VIII Echinoidea (II) Calcutta: Order of the Trustees of the Indian Museum 1922.

52. Cuénot L: Études morphologiques sur les échinodermes. Archives de Biologie (Paris, Bruxelles) 1891, 11:303-680.

53. Koehler R: Echinoderma I: Asteroidea, Ophiuroidea et Echinoidea. In Beiträge zur Kenntnis der Meeresfauna Westafrikas. Edited by: Michaelsen W. Hamburg: Friederichsen 1914:127-303.

54. Mitchell BP: Rediscovery of Gregory`s diverticulum in the scutellid sand dollars. MSc thesis. Western Washington University 1972.

55. Reisman AW: The histology and anatomy of the intestinal tract of Dendraster excentricus, a clypeasteroid echinoid. PhD thesis University of California Los Angeles 1964.

56. Chia FS: Sand dollar: a weight belt for the juvenile. Science 1973, 181:73-74.

57. Timko PL: Sand dollars as suspension feeders: a new description of feeding in Dendraster excentricus. Biological Bulletin 1976, 151:247-259.

58. Chia FS: Selection, storage and elimination of heavy sand particles by the juvenile sand dollar, Dendraster excentricus (Eschscholtz). In Proceedings of the 5th International Echinoderm Conference; Galway. Edited by: Keegan BF, O'Connor BDS. Rotterdam: A.A. Balkema; 1984:215-221.

59. Mooi $R$, Chen CP: Weight belts, diverticula, and the phylogeny of the sand dollars. Bulletin of Marine Sciences 1996, 58:186-195.

60. Agassiz L: Des Scutelles. In Livraison des Monographies d'Échinodermes vivants et fossiles. Edited by: Agassiz L. Neuchâtel: Petitpierre; 1841:1-218.

61. Fox R: Mellita quinquiesperforata. Sand dollar. Invertebrate Anatomy 2001 [http://webs.lander.edu/rsfox/invertebrates/mellita.html].

62. Mortensen T: Echinoidea, Part 2. The Danish Ingolf-Expedition 4 1907, $1-200$.

63. Koehler R: Échinides du Musée Indien à Calcutta. I. Spatangidés. Echinoderma of the Indian Museum, Part VIII Echinoidea (I) Calcutta: Order of the Trustees of the Indian Museum 1914.

64. Chesher RH: The systematics of sympatric species in West Indian spatangoids: a revision of the genera Brissopsis, Plethotaenia, Paleopneustes, and Saviniaster. Studies in Tropical Oceanography 1968, 7:1-168.

65. Kaburek M, Hilgers $H$ : The axial-hydrocoel complex of the endemic Mediterranean heart urchin Schizaster canaliferus (Echinoida: Spatangoida). In Echinoderm Research 1998. Edited by: Candia Carnevali D, Bonasoro F. Rotterdam: A.A. Balkema; 1999:15-20.

66. Hamann O: Beiträge zur Histologie der Echinodermen, Heft 3. Anatomie und Histologie der Echiniden und Spatangiden. Jenaische Zeitschrift für Naturwissenschaften 1887, 21:87-266.

67. Chesher $\mathrm{RH}$ : Contributions to the biology of Meoma ventricosa (Echinoidea: Spatangoida). Bulletin of Marine Science 1969, 19:72-110.

68. De Ridder C, Jangoux M, De Vos L: Description and significance of a peculiar intradigestive symbiosis between bacteria and a depositfeeding echinoid. Journal of Experimental Marine Biology and Ecology 1985, 91:65-76.
69. Wagner J: Anatomie des Palaeopneustes niasicus. In Wissenschaftliche Ergebnisse der Deutschen Tiefsee-Expedition auf dem Dampfer "Valdivia" 18981899. Fünfter Band, erste Lieferung. Edited by: Chun C. Jena: Fischer; 1903:.

70. Gladfelter WB: General ecology of the cassiduloid urchin Cassidulus caribbearum. Marine Biology 1978, 47:149-160.

71. Mortensen T: IV, 2. Clypeasteroida. Clypeasteridae, Arachnoidae, Fibulariidae, Laganidae and Scutellidae. A Monograph of the Echinoidea Copenhagen: C. A. Reitzel 1948

72. Mooi R, Pimentel F: From large to small to large: phylogenetic systematics of rotulid "sand dollars" and their tiny relatives. Integrative and Comparative Biology 2005, 45:1047.

73. Roman J: Morphologie et évolution des Echinolampas (Echinides Cassiduloides). Mémoires du Muséum National d'Histoire Naturelle, Série C 1965, 15:1-341.

74. Mooi R: Paedomorphosis, Aristotle's lantern, and the origin of the sand dollars (Echinodermata: Clypeasteroida). Paleobiology 1990, 16:25-48.

75. Suter S: Cladistic analysis of cassiduloid echinoids: trying to see the phylogeny for the trees. Biological Journal of the Linnean Society 1994, 53:31-72.

76. Kroh A, Smith AB: The phylogeny and classification of post-Palaeozoic echinoids. Journal of Systematic Palaeontology 2010, 8:147-212.

77. Jensen M: Morphology and classification of Euechinoidea Bronn, 1860 a cladistic analysis. Videnskabelige Meddelelser fra Dansk Naturhistorisk Forening i Kjobenhavn 1982, 143:7-99.

78. Jensen M: Functional morphology and systematics of spatangoids (Euechinoidea). In Echinoderm Biology. Edited by: Burke RD, Mladenov PV, Lambert P. Rotterdam: A.A. Balkema; 1988:327-335.

79. Littlewood DTJ, Smith AB: A combined morphological and molecular phylogeny for sea urchins (Echinoidea: Echinodermata). Philosophical Transactions of the Royal Society B Biological Sciences 1995, 347:213-234.

80. Ax P: Echinodermata. In Multicellular animals. Edited by: Ax P. Heidelberg: Springer; 2003:3.

81. Mooi R, Constable H, Lockhart S, Pearse J: Echinothurioid phylogeny and the phylogenetic significance of Kamptosoma (Echinoidea: Echinodermata). Deep-Sea Research I/ 2004, 51:1903-1919.

82. Smith AB: The Echinoid Directory.[http://www.nhm.ac.uk/palaeontology/ echinoids].

83. Stockley B, Smith AB, Littlewood T, Lessios HA, Mackenzie-Dodds J: Phylogenetic relationships of spatangoid sea urchins (Echinoidea): taxon sampling density and congruence between morphological and molecular estimates. Zoologica Scripta 2005, 34:447-468.

84. Smith AB, Pisani D, Mackenzie-Dodds JA, Stockley B, Webster BL, Littlewood DTJ: Testing the molecular clock: molecular and paleontological estimates of divergence times in the Echinoidea (Echinodermata). Molecular Biology and Evolution 2006, 23:1832-1851.

85. Ziegler A, Bartolomaeus T, Mueller S: Sea urchin (Echinoidea) anatomy revealed by magnetic resonance imaging and $3 D$ visualization. In Echinoderms: Durham. Edited by: Harris LG, Boettger SA, Walker CW, Lesser MP. Boca Raton: CRC Press; 2010:305-310.

86. Ziegler A, Mueller S: Analysis of freshly fixed and museum invertebrate specimens using high-resolution, high-throughput MRI. In In vivo NMR imaging: methods and protocols. Edited by: Faber C, Schröder L. New York: Humana Press; .

87. Ziegler A, Ogurreck M, Steinke T, Beckmann F, Prohaska S, Ziegler A Opportunities and challenges for digital morphology. Biology Direct 2010, 5:45.

88. Ruthensteiner B, Hess M: Embedding 3D models of biological specimens in PDF publications. Microscopy Research and Technique 2008, 71:778-786.

89. Kumar P, Ziegler A, Ziegler J, Uchanska-Ziegler B, Ziegler A: Grasping molecular structures through publication-integrated 3D models. Trends in Biochemical Sciences 2008, 33:408-412.

90. Kumar P, Ziegler A, Grahn A, Hee CS, Ziegler A: Leaving the structural ivory tower, assisted by 3D PDF. Trends in Biochemical Sciences 2010, 35:419-422.

doi:10.1186/1471-2148-10-313

Cite this article as: Ziegler et al.: Origin and evolutionary plasticity of the gastric caecum in sea urchins (Echinodermata: Echinoidea). BMC Evolutionary Biology 2010 10:313. 